\title{
The geometry of spinors and the multicomponent BKP and DKP hierarchies
}

\author{
Victor Kac* \\ Department of Mathematics, \\ Massachusetts Institute of Technology \\ Cambridge, MA 02139 \\ USA
}

\author{
Johan van de Leur ${ }^{\dagger}$ \\ Faculty of Applied Mathematics, \\ University of Twente \\ P.O. Box 217, 7500 AE Enschede \\ The Netherlands
}

\begin{abstract}
We develop a formalism of multicomponent BKP hierarchies using elementary geometry of spinors. The multicomponent KP and the modified KP hierarchy (hence all their reductions like KdV, NLS, AKNS or DS) are reductions of the multicomponent BKP.
\end{abstract}

\section{Introduction}

The remarkable connection between the KP hierarchy and representation theory was discovered in the early 1980's by Sato [15] and further developed by Date, Jimbo, Kashiwara and Miwa [3], [4] using the spinor formalism and the classical boson-fermion correspondence. We will now describe this in some detail.

Consider the vector space $V^{\prime}=V^{+} \oplus V^{-}$, where $V^{ \pm}=\bigoplus_{i \in \mathbb{Z}+\frac{1}{2}} \mathbb{C} \psi_{i}^{ \pm}$with symmetric bilinear form

$$
\left(\psi_{i}^{ \pm}, \psi_{j}^{ \pm}\right)=0, \quad\left(\psi_{i}^{ \pm}, \psi_{j}^{\mp}\right)=\delta_{i,-j} .
$$

Let $C \ell V^{\prime}$ be the associated Clifford algebra with relations $\left(u, v \in V^{\prime}\right)$ :

$$
u v+v u=(u, v) 1 .
$$

Consider its spin representation in a vector space $F$ with vacuum vector $|0\rangle$ such that

$$
\psi_{j}^{ \pm}|0\rangle=0 \quad \text { for } j>0 .
$$

By introducing the notion of charge as follows:

$$
\text { charge }|0\rangle=0, \quad \text { charge } \psi_{j}^{ \pm}= \pm 1,
$$

the space $F$ decomposes into charge sectors

$$
F=\bigoplus_{k \in \mathbb{Z}} F_{k}
$$

\footnotetext{
${ }^{*}$ VK is partially supported by NSF grant DMS-9622870.

${ }^{\dagger} \mathrm{JvdL}$ is financially supported by the Netherlands Organization for Scientific Research (NWO).
} 
The group $\overline{\text { Spin } V^{\prime}}$ generated by all elements of the form $1+a \psi_{i}^{+} \psi_{j}^{-}(a \in \mathbb{C})$ acts on $F$ and preserves the charge sectors.

The operators

$$
S^{ \pm}=\sum_{j \in \mathbb{Z}+\frac{1}{2}} \psi_{j}^{ \pm} \otimes \psi_{-j}^{\mp}
$$

commute with the diagonal action of $\overline{\text { Spin } V^{\prime}}$ on $F \otimes F$. Since $S^{ \pm}(|0\rangle \otimes|0\rangle)=0$, an element $\tau$ of $\overline{O_{0}}=\overline{\text { Spin } V^{\prime}}|0\rangle$, the group orbit of the vacuum vector, satisfies equations

$$
S^{ \pm}(\tau \otimes \tau)=\sum_{j \in \mathbb{Z}+\frac{1}{2}} \psi_{j}^{ \pm} \tau \otimes \psi_{-j}^{\mp} \tau=0
$$

One can show that equation $(1.4)_{+}$and $(1.4)$ - are equivalent and that the converse also holds: if $\tau \neq 0$ satisfies (1.4) $)_{+}$, then $\tau \in \overline{O_{0}}$ (see [10] for a proof). The same holds for (1.4) - . Each of the equivalent equations $(1.4)_{+}$and (1.4) $)_{-}$is called the KP hierarchy.

The orbit $\overline{O_{0}}$ can be identified with an algebraic version of Sato's infinite Grassmannian. Let for $k \in \mathbb{Z}, U_{k}^{ \pm}=\bigoplus_{j>k} \mathbb{C} \psi_{j}^{ \pm}$, the Grassmannian $G r^{ \pm}$consists of all subspaces $W^{ \pm} \subset$ $V^{ \pm}$such that for all $k>>0 U_{k}^{ \pm} \subset W^{ \pm}$and $\operatorname{dim} W^{ \pm} / U_{k}^{ \pm}=k$. Let $\tau \in \overline{O_{0}}$, then one can identify the corresponding $W_{\tau}^{ \pm} \in G r^{ \pm}$as follows;

$$
W_{\tau}^{ \pm}=\left\{v \in V^{ \pm} \mid v \tau=0\right\} .
$$

Recall the classical boson-fermion correspondence [10]. Consider the following generating series, called charged fermionic fields:

$$
\psi^{ \pm}(z)=\sum_{i \in \frac{1}{2}+\mathbb{Z}} \psi_{i}^{ \pm} z^{-i-\frac{1}{2}} .
$$

Then we have:

$$
\psi^{\lambda}(y) \psi^{\mu}(z)+\psi^{\mu}(z) \psi^{\lambda}(y)=\delta_{\lambda,-\mu} \delta(y-z), \quad \lambda, \mu= \pm,
$$

where

$$
\delta(y-z)=y^{-1} \sum_{n \in \mathbb{Z}}\left(\frac{y}{z}\right)^{n} .
$$

We split up the fields $\psi^{ \pm}(z)$ in its positive and negative part:

$$
\begin{array}{r}
\psi^{ \pm}(z)=\psi^{ \pm}(z)_{+}+\psi^{ \pm}(z)_{-}, \quad \text { where } \\
\psi^{ \pm}(z)_{+}=\sum_{i \in \mathbb{Z}_{+}} \psi_{-i-\frac{1}{2}}^{ \pm} z^{i} \text { and } \psi^{ \pm}(z)_{-}=\psi^{ \pm}(z)-\psi^{ \pm}(z)_{+},
\end{array}
$$

so that

$$
\psi^{ \pm}(z)|0\rangle=\psi^{ \pm}(z)_{+}|0\rangle .
$$

Define the bosonic fields

$$
\alpha(z)=\sum_{k \in \mathbb{Z}} \alpha_{k} z^{-k-1}=: \psi^{+}(z) \psi^{-}(z):
$$

where the normally ordered product is defined, as usual, by

$$
: \psi^{\lambda}(y) \psi^{\mu}(z):=\psi^{\lambda}(y)_{+} \psi^{\mu}(z)-\psi^{\mu}(z) \psi^{\lambda}(y)_{-} .
$$


Then one has (using Wick's formula):

$$
\begin{aligned}
{\left[\psi^{\lambda}(y), \alpha(z)\right] } & =-\lambda \delta(y-z), \quad \lambda= \pm, \\
{[\alpha(y), \alpha(z)] } & =\delta_{z}^{\prime}(y-z) .
\end{aligned}
$$

Thus, the $\alpha_{m}$ form the oscillator algebra,

$$
\left[\alpha_{m}, \alpha_{n}\right]=m \delta_{m,-n}, \text { with } \alpha_{k}|0\rangle=0 \text { for } k \geq 0 .
$$

The operator $\alpha_{0}$ is diagonalizable in $F$, with eigenspaces the charge sectors $F_{k}$, i.e. $\alpha_{0} f_{k}=$ $k f_{k}$ for $f_{k} \in F_{k}$. For that reason we call $\alpha_{0}$ the charge operator.

In order to express the fermionic fields $\psi^{\lambda}(z)$ in terms of the oscillator algebra, we need an additional operator $Q$ on $F$ defined by

$$
Q|0\rangle=\psi_{-\frac{1}{2}}^{+}|0\rangle, \quad Q \psi_{k}^{+}=\psi_{k \mp 1}^{ \pm} Q .
$$

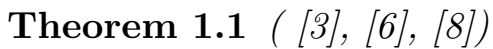

$$
\psi^{ \pm}(z)=Q^{ \pm 1} z^{ \pm \alpha_{0}} \exp \left(\mp \sum_{k<0} \frac{\alpha_{k}}{k} z^{-k}\right) \exp \left(\mp \sum_{k>0} \frac{\alpha_{k}}{k} z^{-k}\right) .
$$

We now identify $F$ with the space $B=\mathbb{C}\left[q, q^{-1}, t_{1}, t_{2}, \ldots\right]$ via the vector space homorphism

$$
\sigma: F \stackrel{\sim}{\rightarrow} B
$$

given by

$$
\sigma\left(\alpha_{-m_{1}} \ldots \alpha_{-m_{s}} Q^{k}|0\rangle\right)=m_{1} m_{2} \ldots m_{s} t_{m_{1}} t_{m_{2}} \ldots t_{m_{s}} q^{k} .
$$

The transported charge is as follows

$$
\text { charge }\left(p(t) q^{k}\right)=k,
$$

and the transported charge decomposition is

$$
B=\bigoplus_{m \in \mathbb{Z}} B_{m}, \text { where } B_{m}=\mathbb{C}\left[t_{1}, t_{2} \ldots\right] q^{m} .
$$

The transported action of the operators $\alpha_{m}$ and $Q$ on $B$ is as follows:

$$
\begin{aligned}
\sigma \alpha_{-m} \sigma^{-1}\left(p(t) q^{k}\right) & =m t_{m} p(t) q^{k}, \\
\sigma \alpha_{m} \sigma^{-1}\left(p(t) q^{k}\right) & =\frac{\partial p(t)}{\partial t_{m}} q^{k}, \\
\sigma \alpha_{0} \sigma^{-1}\left(p(t) q^{k}\right) & =q \frac{\partial}{\partial q}\left(p(t) q^{k}\right)=k p(t) q^{k}, \\
\sigma Q \sigma^{-1}\left(p(t) q^{k}\right) & =p(t) q^{k+1} .
\end{aligned}
$$

Hence

$$
\sigma \psi^{ \pm}(z) \sigma^{-1}=q^{ \pm 1} z^{ \pm q \frac{\partial}{\partial q}} e^{ \pm \xi(t, z)} e^{\mp \eta(t, z)}
$$

where

$$
\xi(t, z)=\sum_{k=1}^{\infty} t_{k} z^{k} \quad \text { and } \quad \eta(t, z)=\sum_{k=1}^{\infty} \frac{1}{k} \frac{\partial}{\partial t_{k}} z^{-k}
$$


We now rewrite equations $(1.4)_{ \pm}$in the bosonic picture. First notice that $(1.4)_{ \pm}$is equivalent to

$$
\operatorname{Res}_{z=0} d z \psi^{ \pm}(z) \tau \otimes \psi^{\mp}(z) \tau=0 .
$$

Here and further $\operatorname{Res}_{z=0} d z \sum f_{i} z^{i}$, where the $f_{i}$ are independent of $z$, stands for $f_{-1}$. Now think of $B_{0} \otimes B_{0}$ as the polynomial algebra $\mathbb{C}\left[t_{1}^{\prime}, t_{1}^{\prime \prime}, t_{2}^{\prime}, t_{2}^{\prime \prime}, \ldots\right]$. Then applying $\sigma$ to both sides of $(1.9)_{ \pm}$, we obtain a system of equations describing the orbit $\sigma\left(\overline{O_{0}}\right)$ :

$$
\operatorname{Res}_{z=0} d z e^{\mp \eta\left(t^{\prime}, z\right)} \tau\left(t^{\prime}\right) e^{ \pm \xi\left(t^{\prime}, z\right)} e^{ \pm \eta\left(t^{\prime \prime}, z\right)} \tau\left(t^{\prime \prime}\right) e^{\mp \xi\left(t^{\prime \prime}, z\right)}=0
$$

Divide $(1.10)_{ \pm}$by $\tau\left(t^{\prime}\right) \tau\left(t^{\prime \prime}\right)$, one obtains the bilinear identity

$$
\operatorname{Res}_{z=0} d z \Psi^{ \pm}\left(t^{\prime}, z\right) \Psi^{\mp}\left(t^{\prime \prime}, z\right)=0,
$$

for the $\mathrm{KP}$ wave function $\Psi^{+}(t, z)$ and its adjoint $\Psi^{-}(t, z)$ defined by

$$
\Psi^{ \pm}(t, z)=\frac{e^{\mp \eta(t, z)} \tau(t)}{\tau(t)} e^{ \pm \xi(t, z)}
$$

Instead of dividing by $\tau\left(t^{\prime}\right) \tau\left(t^{\prime \prime}\right)$, one can make a change of variables

$$
t_{k}=\frac{1}{2}\left(t_{k}^{\prime}+t_{k}^{\prime \prime}\right), \quad s_{k}=\frac{1}{2}\left(t_{k}^{\prime}-t_{k}^{\prime \prime}\right)
$$

Then $(1.10) \pm$ becomes

$$
\operatorname{Res}_{z=0} d z e^{ \pm \xi(2 s, z)} e^{\mp \eta(s, z)} \tau(s+t) \tau(t-s)=0 .
$$

Introducing the elementary Schur functions $P_{k}(t), k \in \mathbb{Z}$ by

$$
\sum_{k \in \digamma} P_{k}(t) z^{k}=e^{\xi(t, z)}
$$

we rewrite $(1.12) \pm$ as follows

$$
\sum_{j=0}^{\infty} P_{j}( \pm 2 s) P_{j+1}\left(\mp \frac{\tilde{\partial}}{\partial s}\right) \tau(s+t) \tau(t-s)=0,
$$

where

$$
\frac{\tilde{\partial}}{\partial t}=\left(\frac{\partial}{\partial t_{1}}, \frac{1}{2} \frac{\partial}{\partial t_{2}}, \frac{1}{3} \frac{\partial}{\partial t_{3}}, \ldots\right) .
$$

Using Taylors formula we rewrite $(1.14)_{ \pm}$once more:

$$
\left.\sum_{j=0}^{\infty} P_{j}( \pm 2 s) P_{j+1}\left(\mp \frac{\tilde{\partial}}{\partial u}\right) e^{\sum_{r=1}^{\infty} s_{r} \frac{\partial}{\partial u_{r}}} \tau(t+u) \tau(t-u)\right|_{u=0}=0
$$

which is a generating series of Hirota bilinear equations for the KP hierarchy:

$$
\sum_{j=0}^{\infty} P_{j}( \pm 2 s) P_{j+1}(\mp \tilde{D}) e^{\sum_{r=1}^{\infty} s_{r} D_{r}} \tau \cdot \tau=0 .
$$


Recall that for a polynomial $p$, the corresponding Hirota bilinear equation on $\sigma$ and $\rho$ is

$$
p(D) \sigma \cdot \rho=\left.p\left(\frac{\partial}{\partial u}\right) \sigma(t+u) \rho(t-u)\right|_{u=0}=0 .
$$

Calculating the coefficient of $s_{3}$ in $(1.16)+$ one obtains

$$
\left(D_{1}^{4}-4 D_{1} D_{3}+3 D_{2}^{2}\right) \tau \cdot \tau=0 .
$$

Putting $x=t_{1}, y=t_{2}, t=t_{3}$ and $u(x, y, t)=\left(2 \log \tau\left(x, y, t, t_{4}, t_{5}, \ldots\right)\right)_{x x}$, where $t_{4}, t_{5}, \ldots$ are viewed as parameters, then (1.17) turns into

$$
\frac{3}{4} u_{y y}=\left(u_{t}-\frac{3}{2} u u_{x}-\frac{1}{4} u_{x x x}\right)_{x}
$$

which is the classical Kadomtsev-Petviashvili (KP)equation (hence the name KP hierarchy).

We return now to $\tau \in \overline{O_{0}}$. Instead of considering only $W_{\tau}^{+} \in G r^{+}$or $W_{\tau}^{-} \in G r^{-}$one could consider $W_{\tau}=W_{\tau}^{+} \oplus W_{\tau}^{-}$. Notice that this is a maximal isotropic subspace of $V^{\prime}$. So instead of considering $\mathrm{Gr}^{+}$one could consider another Grassmannian $\mathrm{Gr}$, consisting of all maximal isotropic subspaces $W \subset V^{\prime}$ such that $U_{k}^{ \pm} \subset W$ for $k>>0$. This Grassmannian should parametrize some other group orbit. In order to describe this group, it will be convenient to add one more basis element to $V^{\prime}$. Let $V=V^{\prime} \oplus \mathbb{C} \psi_{0}$, with bilinear form given by (1.1) and

$$
\left(\psi_{0}, V^{\prime}\right)=0, \quad\left(\psi_{0}, \psi_{0}\right)=1 .
$$

Let $C \ell V$ be the corresponding Clifford algebra, with relations (1.2), but now for $u, v \in V$. The spin representation of $C \ell V$ in the vector space $F$ is given by (1.3) and

$$
\psi_{0}|0\rangle=-\frac{1}{\sqrt{2}}|0\rangle .
$$

Since $\psi_{0}^{2}=\frac{1}{2}$, the eigenvalues of $\psi_{0}$ on $F$ are $-\frac{1}{\sqrt{2}}$ and $\frac{1}{\sqrt{2}}$. Let $F_{\overline{0}}$ and $F_{\overline{1}}$ be the corresponding eigenspaces. Then $F_{\bar{\nu}}=\bigoplus_{k \in \nu+2 \mathbb{Z}} F_{k}$. Let Spin $V$ (resp Spin $V^{\prime}$ ) be the spin group generated by all invertible elements of the form $1+a u v, a \in \mathbb{C}, u, v \in V$ (resp. $u, v \in V^{\prime}$ ) (see Section 2 for more details). Then clearly $F$ (resp. $F_{\bar{\nu}}$ ) is a Spin $V$ - (resp Spin $V^{\prime}$-)module. Let $|1\rangle=\psi_{-\frac{1}{2}}|0\rangle$ and let $O=\operatorname{Spin} V|0\rangle$, (resp. $\left.O_{\bar{\nu}}=\operatorname{Spin} V^{\prime}|\nu\rangle\right)$ be the Spin $V$ - (resp Spin $\left.V^{\prime}-\right)$ orbit of $|0\rangle$ (resp. $\left.|\nu\rangle\right)$. Then $O_{\bar{\nu}} \subset O \cap F_{\bar{\nu}}$.

To a $\tau \in O$ there corresponds a point $W_{\tau} \in G r$ given by

$$
W_{\tau}=\{v \in V \mid v \tau=0\},
$$

called the annihilator space of $\tau$. Let

$$
S^{\prime}=S^{+}+S^{-} \text {and } S=\psi_{0} \otimes \psi_{0}+S^{\prime} .
$$

One of the main observations of this paper is the following theorem, proved in $\S 2$ using some simple arguments on geometry of spinors:

Theorem 1.2 (a) If $\tau \in F$ and $\tau \neq 0$, then $\tau \in O$ if only if $\tau$ satisfies the equation

$$
S(\tau \otimes \tau)=\frac{1}{2} \tau \otimes \tau .
$$


(b) If $\tau \in F_{\bar{\nu}}(\nu \in \mathbb{Z} / 2 \mathbb{Z})$ and $\tau \neq 0$, then $\tau \in O_{\bar{\nu}}$ if and only if $\tau$ satisfies the equation

$$
S^{\prime}(\tau \otimes \tau)=0 .
$$

Equation (1.18) (resp. (1.19)) is called the fermionic BKP (resp. DKP) hierarchy. As a consequence we obtain

Corollary 1.1 Let $\tau \in F_{k}(k \in \mathbb{Z})$, then $\tau$ satisfies the KP hierarchy,i.e. equation $(1.4)_{ \pm}$, if and only if $\tau$ satisfies the DKP (or BKP) hierarchy.

If we split up $\tau \in O$ into eigenspaces for $\psi_{0}$, i.e., $\tau=\tau_{0}+\tau_{1}$ with $-\sqrt{2} \psi_{0} \tau_{\nu}=(-)^{\nu} \tau_{\nu}$, then one has

Theorem 1.3 Let $\tau \in F, \tau=\tau_{0}+\tau_{1}$, where $\tau_{\nu} \in F_{\bar{\nu}}$. Then $\tau$ satisfies the fermionic BKP hierarchy if and only if both $\tau_{0}$ and $\tau_{1}$ satisfy the DKP hierarchy (1.19) and the Modified DKP hierarchy:

$$
S^{\prime}\left(\tau_{0} \otimes \tau_{1}\right)=\tau_{1} \otimes \tau_{0}
$$

As in the case of the KP hierarchy, we will again use the boson-fermion correspondence to rewrite the BKP hierarchy in the bosonic picture. Notice first that (1.18) is equivalent to

$$
\operatorname{Res}_{z=0} d z \frac{1}{z} \psi_{0} \tau \otimes \psi_{0} \tau+\psi^{+}(z) \tau \otimes \psi^{-}(z) \tau-\psi^{-}(-z) \tau \otimes \psi^{+}(-z) \tau=\frac{1}{2} \tau \otimes \tau \text {. }
$$

Now apply $\sigma$ to (1.21). Writing $\sigma(\tau)=\sum_{n \in \mathbb{Z}} \tau_{n}(t) q^{n}$, we obtain

$$
\begin{array}{rl}
\operatorname{Res}_{z}=0 & d z \sum_{n, m \in \mathbb{Z}} \frac{1}{2 z}(-)^{n} \tau_{n}\left(t^{\prime}\right)\left(q^{\prime}\right)^{n}(-)^{m} \tau_{m}\left(t^{\prime \prime}\right)\left(q^{\prime \prime}\right)^{m} \\
& +z^{n} e^{\xi\left(t^{\prime}, z\right)} e^{-\eta\left(t^{\prime}, z\right)} \tau_{n}\left(t^{\prime}\right)\left(q^{\prime}\right)^{n+1} z^{-m} e^{-\xi\left(t^{\prime \prime}, z\right)} e^{\eta\left(t^{\prime \prime}, z\right)} \tau_{m}\left(t^{\prime \prime}\right)\left(q^{\prime \prime}\right)^{m-1} \\
& -(-z)^{-n} e^{-\xi\left(t^{\prime},-z\right)} e^{\eta\left(t^{\prime},-z\right)} \tau_{n}\left(t^{\prime}\right)\left(q^{\prime}\right)^{n-1}(-z)^{m} e^{\xi\left(t^{\prime \prime},-z\right)} e^{-\eta\left(t^{\prime \prime},-z\right)} \tau_{m}\left(t^{\prime \prime}\right)\left(q^{\prime \prime}\right)^{m+}(1 . \\
& =\frac{1}{2} \sum_{n, m \in \mathbb{Z}} \tau_{n}\left(t^{\prime}\right)\left(q^{\prime}\right)^{n} \tau_{m}\left(t^{\prime \prime}\right)\left(q^{\prime \prime}\right)^{m}
\end{array}
$$

Taking the coefficient of $\left(q^{\prime}\right)^{n}\left(q^{\prime \prime}\right)^{m}$, we find for all $n, m \in \mathbb{Z}$ the following equation:

$$
\begin{aligned}
\operatorname{Res}_{z} & =0 \\
& +z^{n-1} e^{\xi\left(t^{\prime}, z\right)} e^{-\eta\left(t^{\prime}, z\right)} \tau_{n-1}\left(t^{\prime}\right) z^{-m-1} e^{-\xi\left(t^{\prime \prime}, z\right)} e^{\eta\left(t^{\prime \prime}, z\right)} \tau_{m+1}\left(t^{\prime \prime}\right) \\
& -(-z)^{-n-1} e^{-\xi\left(t^{\prime},-z\right)} e^{\eta\left(t^{\prime},-z\right)} \tau_{n+1}\left(t^{\prime}\right)(-z)^{m-1} e^{\xi\left(t^{\prime \prime},-z\right)} e^{-\eta\left(t^{\prime \prime},-z\right)} \tau_{m-1}\left(t^{\prime \prime}\right) \\
& =\frac{1}{2} \tau_{n}\left(t^{\prime}\right) \tau_{m}\left(t^{\prime \prime}\right)
\end{aligned}
$$

Now divide by $\tau_{p}\left(t^{\prime}\right) \tau_{q}\left(t^{\prime \prime}\right)$ for $p=n-1, n, n+1, q=m-1, m, m+1$ and view the obtained result as products of rows and columns of $3 \times 3$-matrices. In other words, let

$$
\begin{aligned}
& S(z)=\operatorname{diag}\left(-1, \frac{1}{2 z}, 1\right), \\
& Q^{ \pm}(t, z)=\operatorname{diag}\left(e^{\mp \xi(t, z)}, 1, e^{ \pm \xi(t, z)}\right), \\
& R^{ \pm}(n, z)=\operatorname{diag}\left((-z)^{\mp n},(-)^{n}, z^{ \pm n}\right),
\end{aligned}
$$




$$
\begin{gathered}
P^{ \pm}(n, t, z)=\frac{1}{\tau_{n}(t)}\left(\begin{array}{ccc}
e^{ \pm \eta(t,-z)} \tau_{n}(t) & -\tau_{n \mp 1}(t) & z^{-2} e^{\mp \eta(t, z)} \tau_{n \mp 2}(t) \\
(-z)^{-1} e^{ \pm \eta(t,-z)} \tau_{n \pm 1}(t) & \tau_{n}(t) & z^{-1} e^{\mp \eta(t, z)} \tau_{n \mp 1}(t) \\
z^{-2} e^{ \pm \eta(t,-z)} \tau_{n \pm 2}(t) & -\tau_{n \pm 1}(t) & e^{\mp \eta(t, z)} \tau_{n}(t)
\end{array}\right) \\
T^{ \pm}(n, t, z)=\left(\begin{array}{ccc}
1 & \tau_{n \mp 1} / \tau_{n}(t) & 0 \\
0 & 1 & 0 \\
0 & \tau_{n \pm 1} / \tau_{n}(t) & 1
\end{array}\right)
\end{gathered}
$$

and put

$$
\Psi^{ \pm}(n, t, z)=P^{ \pm}(n, t, z) R^{ \pm}(n, z) Q^{ \pm}(t, z),
$$

then $(1.23)$ is equivalent to

$$
\operatorname{Res}_{z=0} d z \Psi^{+}\left(n, t^{\prime}, z\right) S(z){ }^{t} \Psi^{-}\left(m, t^{\prime \prime}, z\right)=\operatorname{Res}_{z=0} d z T^{+}\left(n, t^{\prime}, z\right) S(z){ }^{t} T^{-}\left(m, t^{\prime \prime}, z\right) .
$$

We can rewrite this once more to get a nicer formula. Define BKP wave functions by

$$
\Phi^{ \pm}(n, t, z)=T^{ \pm}(n, t, z)^{-1} \Psi^{ \pm}(n, t, z),
$$

then $(1.28)$ turns into

$$
\operatorname{Res}_{z=0} d z \Phi^{+}\left(n, t^{\prime}, z\right) S(z){ }^{t} \Phi^{-}\left(m, t^{\prime \prime}, z\right)=\operatorname{diag}\left(0, \frac{1}{2}, 0\right) .
$$

From the formulas (1.25), (1.26) and $(1.29)$, it is easy to determine that $\Phi^{-}(n, t, z)$ is completely determined by $\Phi^{+}(n, t, z)$. Using the bilinear identity $(1.30)$ one can in fact show that $\Phi^{+}(n, t, z)$ determines its neighbors $\Phi^{+}(n \pm 1, t, z)$. The DKP bilinear identity on the wave functions is obtained from (1.30) by removing the second row and column from all matrices (1.24)-(1.26).

Differentiate (1.30) by $t_{\ell}^{\prime}$, then using Lemma 5.1 of this paper, we obtain the Sato equation of this BKP hierarchy. Fix $n \in \mathbb{Z}$, let $\partial=\frac{\partial}{\partial t_{1}}$ and let

$$
\begin{aligned}
& N(\ell, \partial)=\operatorname{diag}\left(-(-\partial)^{\ell}, 0, \partial^{\ell}\right), \\
& K(\partial)=T^{+}(n, t, \partial)^{-1} P^{+}(n, t, \partial)=\sum_{j=0}^{\infty} K^{(j)} \partial^{-j}
\end{aligned}
$$

then $K_{i 2}^{(j)}=0$ for $i=1,2,3$ and $j=1,2, \ldots$ One finds the following Sato equation:

$$
\frac{\partial K(\partial)}{\partial t_{\ell}}=-\left(K(\partial) N(\ell, \partial) K(\partial)^{-1} S(\partial)\right)_{+} S(\partial)^{-1} K(\partial)
$$

We now return to equation (1.23) and rewrite it into a generating series of Hirota bilinear equations. Make the same change of variables as before, viz. (1.10) and use the elementary Schur functions defined by (1.13). Then $(1.23)$ is equivalent to

$$
\begin{gathered}
\frac{1}{2}\left((-)^{n+m}-1\right) \tau_{n}(s+t) \tau_{m}(t-s)+\sum_{j=0}^{\infty} P_{j}(2 s) P_{j+n-m-1}\left(-\frac{\tilde{\partial}}{\partial s}\right) \tau_{n-1}(s+t) \tau_{m+1}(t-s)+ \\
P_{j}(-2 s) P_{j+m-n-1}\left(\frac{\tilde{\partial}}{\partial s}\right) \tau_{n+1}(s+t) \tau_{m-1}(t-s)=0 .
\end{gathered}
$$


Using Taylor's formula this turns into the following generating series of Hirota bilinear equations:

$$
\begin{gathered}
\frac{1}{2}\left((-)^{n+m}-1\right) e^{\sum_{r=1}^{\infty} s_{r} D_{r}} \tau_{n} \cdot \tau_{m}+\sum_{j=0}^{\infty} P_{j}(2 s) P_{j+n-m-1}(-\tilde{D}) e^{\sum_{r=1}^{\infty} s_{r} D_{r}} \tau_{n-1} \cdot \tau_{m+1}+ \\
P_{j}(-2 s) P_{j+m-n-1}(\tilde{D}) e^{\sum_{r=1}^{\infty} s_{r} D_{r}} \tau_{n+1} \cdot \tau_{m-1}=0
\end{gathered}
$$

The simplest Hirota bilinear equations of the charged BKP hierarchy, appear in the constant term when we take $m-n=3$ and 4 ( $m-n=0,1,2$ give trivial equations):

$$
\begin{gathered}
\left(D_{1}^{2}+D_{2}\right) \tau_{n+1} \cdot \tau_{n+2}=2 \tau_{n} \tau_{n+3}, \\
\left(D_{1}^{3}+3 D_{1} D_{2}+2 D_{3}\right) \tau_{n} \cdot \tau_{n+2}=0 .
\end{gathered}
$$

The coefficient of $s_{3}$ with $n-m=2$ gives:

$$
\left(D_{1}^{4}-4 D_{1} D_{3}+3 D_{2}^{2}\right) \tau_{n} \cdot \tau_{n}=24 \tau_{n+2} \tau_{n-2} .
$$

Another BKP hierarchy, which is natural to call the neutral BKP hierarchy, was introduced in [4]. It uses neutral fermions and the neutral boson-fermion correspondence (cf. $\S 3.2$ of the present paper).

In this paper we consider the general situation of $n$ charged fermions and $n^{\prime}$ neutral fermions. Using the multicomponent boson-fermion correspondence we derive the general multicomponent BKP and DKP hierarchies in the form of a system of Hirota bilinear equations and in the form of bilinear equations on the wave functions ( $\S \S 3$ and 4 ). Note that though the BKP hierarchy in the fermionic picture is always the same (eq. (1.18)),

different bosonizations (depending of the choice of the multicomcponent fermions) produce quite different hierarchies of partial differential equations.

As in the case of the $n$-component KP hierarchy (see [11]) we go on to develop the formalism of Sato and Lax equations for the multicomponent BKP hierarchies ( $\$ 5)$. In conclusion we derive a OSASM-type formula ( $\S 6)$, using the method of [19] (see also [11]).

\section{Geometry of spinors}

Let $V$ be a vector space with a symmetric bilinear form $(\cdot, \cdot)$. We shall assume for simplicity that $V$ is at most countable dimensional. Given a non-isotropic vector $\alpha$ (i.e. $(\alpha, \alpha) \neq 0$ ), the associated reflection $r_{\alpha}$ is defined by

$$
r_{\alpha}(v)=v-\frac{2(\alpha, v)}{(\alpha, \alpha)} \alpha
$$

We denote by $O(V)$ the group generated by all $-r_{\alpha}$ and by $S O(V)$ its subgroup consisting of products of an even number of reflections.

Let $C \ell V$ be the associated Clifford algebra, that is the quotient of the tensor algebra over $V$ by the ideal generated by relations

$$
u v+v u=(u, v) 1, \quad \text { where } u, v \in V .
$$

If $\left\{v_{i}\right\}_{i \in J}$ is a basis of $V$ indexed by an ordered set, then the elements

$$
v_{i_{1}} v_{i_{2}} \ldots v_{i_{s}} \text { with } i_{1}<i_{2}<\ldots<i_{s}, \quad s \in \mathbb{Z}_{+},
$$


form a basis of $C \ell V$. We have a $\mathbb{Z} / 2 \mathbb{Z}$-gradation

$$
C \ell V=C \ell_{\overline{0}} V \oplus C \ell_{\overline{1}} V
$$

where $C \ell_{\nu} V(\nu \in \mathbb{Z} / 2 \mathbb{Z})$ is spanned by all products of the form (2.2) with $s \equiv \nu \bmod 2$. Here and further we identify $V$ with its image in $C \ell V$.

Let $(C \ell V)^{\times}$denote the multiplicative group of invertible elements of the algebra $C \ell V$. We denote by Pin $V$ the subgroup of $(C \ell V)^{\times}$generated by all the elements $a$ such that $a V a^{-1}=V$ and let Spin $V=\operatorname{Pin} V \cap C \ell_{\overline{0}} V$.

If $\alpha \in V$ is a non-isotropic vector, then, by (2.1):

$$
\alpha^{-1}=\frac{2 \alpha}{(\alpha, \alpha)},
$$

hence $\alpha \in(C \ell V)^{\times}$. From (2.1) and (2.3) we obtain

$$
\alpha v \alpha^{-1}=-r_{\alpha}(v),
$$

hence $\alpha \in \operatorname{Pin} V$. We have a homomorphism $T:$ Pin $V \rightarrow O(V), g \mapsto T_{g}$ defined by $(v \in V)$ :

$$
T_{g}(v)=g v g^{-1} \in V \text {. }
$$

Let $U$ be a subspace of $\mathbb{C} 1+V$. Let

$$
F(V, U)=C \ell V /(C \ell V) U
$$

and let $|0\rangle$ denote the image of 1 in $F(V, U)$. The space $F(V, U)$ caries a structure of a $C \ell V$-module induced by left multiplication. This module restricted to $\operatorname{Pin} V$ is called a spin module of the group Pin $V$.

Consider the special case when $V$ has a basis $\left\{v_{i}\right\}_{i \in \mathbb{Z}}$ such that

$$
\left(v_{i}, v_{j}\right)=\delta_{i,-j} \quad i, j \in \mathbb{Z} .
$$

Then $U_{0}:=\sum_{j<0} \mathbb{C} v_{j}$ is a maximal isotropic subspace of the space $V$. Let

$$
U=U_{0}+\mathbb{C}\left(1+\sqrt{2} v_{0}\right)
$$

and consider the $C \ell V$-module $F:=F(V, U)$. Note that

$$
v_{0}|0\rangle=-\frac{1}{\sqrt{2}}|0\rangle \quad \text { and } v_{j}|0\rangle=0 \quad \text { if } j<0 .
$$

Let

$$
V^{\prime}=\left\{w \in V^{0} \mid\left(w, v_{0}\right)=0\right\} .
$$

Then $C \ell V^{\prime}$ is a subalgebra of $C \ell V$ (it is actually the centralizer of $v_{0}$ ) and Pin $V^{\prime}$, Spin $V^{\prime}$ are subgroups of Pin $V$ and Spin $V$. The Spin $V^{\prime}$-module $F$ decomposes in the direct sum of submodules $F_{\overline{0}}$ and $F_{\overline{1}}$, called half spin modules, defined by

$$
F_{\bar{\nu}}=\left\{f \in F \mid-\sqrt{2} v_{0} f=(-)^{\nu} f\right\} .
$$


The $C \ell V$ - (resp. $C \ell V^{\prime}$-)module $F$ is called the spin module of type B (resp. D).

Given $f \in F$, let

$$
\text { Ann } f=\{v \in V \mid v f=0\}
$$

If moreover $f \in F_{\bar{\nu}}$, then

$$
\text { Ann } f=\left\{v \in V^{\prime} \mid v f=0\right\} .
$$

Recall that we have the vacuum vector $|0\rangle \in F$ characterized (up to a constant factor) among the vectors of $F$ by the property that $A n n|0\rangle=U_{0}$. Let $|1\rangle=v_{1}|0\rangle \in F_{\overline{1}}$. Note that $|1\rangle$ is characterized by the property that $A n n|1\rangle=r_{v_{1}+v_{-1}}\left(U_{0}\right)$.

Remark 2.1 It is clear that the $C \ell_{\overline{0}} V$-module $F$ is irreducible. The $C \ell_{\overline{0}} V^{\prime}$-modules $F_{\overline{0}}$ and $F_{\overline{1}}$ are also irreducible and moreover inequivalent. The same holds with $C \ell_{\overline{0}} V$ (resp. $\left.C \ell_{\overline{0}} V^{\prime}\right)$ replaced by Spin $V$ (resp. Spin $\left.V^{\prime}\right)$.

Introduce the following operators on the space $F \otimes F$ :

$$
S^{\prime}=\sum_{0 \neq j \in \mathbb{Z}} v_{j} \otimes v_{-j} \text { and } S=v_{0} \otimes v_{0}+S^{\prime}
$$

Since $v_{j}|0\rangle=v_{j}|1\rangle=0$ for $j\left\langle-1, v_{-1} \mid 0\right\rangle=v_{1}|1\rangle=0$ and $v_{0}|0\rangle=-\frac{1}{\sqrt{2}}|0\rangle, v_{0}|1\rangle=\frac{1}{\sqrt{2}}|1\rangle$ we find $(\epsilon=0$ or 1$)$ :

$$
\begin{aligned}
S^{\prime}(|\epsilon\rangle \otimes|\epsilon\rangle) & =0, \\
S(|0\rangle \otimes|0\rangle) & =\frac{1}{2}|0\rangle \otimes|0\rangle, \\
S^{\prime}(|0\rangle \otimes|1\rangle) & =|1\rangle \otimes|0\rangle, \\
S^{\prime}(|1\rangle \otimes|0\rangle) & =|0\rangle \otimes|1\rangle .
\end{aligned}
$$

Lemma 2.1 The operator $S$ (resp. $S^{\prime}$ ) commutes with the diagonal action of the group Pin $V\left(\right.$ resp. Pin $\left.V^{\prime}\right)$ on $F \otimes F$.

Proof. For $a \in \operatorname{Pin} V$ we have

$$
\begin{aligned}
(a \otimes a) S\left(a^{-1} \otimes a^{-1}\right) & =\sum_{j \in \mathbb{Z}} T_{a}\left(v_{j}\right) \otimes T_{a}\left(v_{-j}\right) \\
& =\sum_{i \in \mathbb{Z}} \sum_{j, k \in \mathbb{Z}} a_{i j} v_{j} \otimes a_{-i k} v_{k}=\sum_{i, j, k \in \mathbb{Z}} a_{i j} a_{-i k} v_{j} \otimes v_{k}=\sum_{j \in \mathbb{Z}} v_{j} \otimes v_{-j},
\end{aligned}
$$

since $\sum_{i} a_{i j} a_{-i k}=\delta_{j,-k}$ (because $T_{a} \in O(V)$ ). The proof for $S^{\prime}$ is analogous.

Let $(\nu=0,1)$ :

$$
O=\operatorname{Spin} V|0\rangle \text { and } O_{\bar{\nu}}=\operatorname{Spin} V^{\prime}|\nu\rangle
$$

be the orbit of the vector $|0\rangle$ (resp. $|\nu\rangle$ ) under the action of the group Spin $V$ (resp. Spin $\left.V^{\prime}\right)$. Then clearly Pin $V|0\rangle=O$ and Pin $V^{\prime}|\nu\rangle=O_{\overline{0}} \cup O_{\overline{1}}$.

Lemma 2.2 If $\tau \in O$ (resp. $\tau \in O_{\bar{\nu}}$ ) and $\alpha \in V$ (resp. $\alpha \in V^{\prime}$ ), then $\alpha \tau \in O \cup\{0\}$ (resp. $\alpha \tau \in O \overline{\nu+1} \cup\{0\})$. 
Proof. For simplicity we consider only the case $\tau \in O_{\bar{\nu}}$. If $\alpha$ is non-isotropic then $\alpha \tau \in O \overline{\nu+1}$ by definition. If $(\alpha, \alpha)=0$ and $\alpha \in($ resp. $\notin)$ Ann $|\nu\rangle$, then $\alpha|\nu\rangle=0$ (resp. there exists a $u \in A n n|\nu\rangle$ such that $(\alpha, u) \neq 0$, so that $\alpha+u$ is non-isotropic, hence $\alpha|\nu>=(\alpha+u)| \nu\rangle \in O \overline{\nu+1})$. Since for some $g \in \operatorname{Spin} V^{\prime}, \tau=g|\nu\rangle$ we have $\alpha \tau=g\left(g^{-1} \alpha g\right)|\nu\rangle=g\left(T_{g^{-1}}(\alpha)\right)|\nu\rangle \in g\left(O_{\overline{\nu+1}} \cup\{0\}\right) \subset O \overline{\nu+1} \cup\{0\}$.

For $k \in \mathbb{Z}$, let $U_{k}=\sum_{j<-k} \mathbb{C} v_{j}$. Introduce the Grassmannians $G r$ and $G r^{\prime}$ as the sets of all maximal isotropic subspaces $W$ of $V$, respectively $V^{\prime}$, such that $W \supset U_{k}$ for $k>>0$.

Remark 2.2 For any $\tau \in O$ (resp. $\tau \in O_{\bar{\nu}}$ ) we have

$$
\text { Ann } \left.\tau \in G r \quad \text { (resp. Ann } \tau \in G r^{\prime}\right) \text {. }
$$

Indeed this is clear if $\tau=|0\rangle$ (resp. $\tau=|\nu\rangle)$, and for $g \in \operatorname{Spin} V\left(g \in \operatorname{Spin} V^{\prime}\right)$ we have

$$
\text { Ann } g \tau=T_{g}(\text { Ann } \tau) .
$$

Lemma 2.3 (a) The group $S O(V)$ (resp. $S O\left(V^{\prime}\right)$ ) is the group of all orthogonal transformations $A$ of the space $V$ (resp. $V^{\prime}$ ) such that the matrix $A-I$ has only finitely many non-zero entries and $\operatorname{det} A=1$.

(b) The homomorphisms $T: \operatorname{Pin} V \rightarrow O(V), T: \operatorname{Pin} V^{\prime} \rightarrow O\left(V^{\prime}\right), T: \operatorname{Spin} V \rightarrow S O(V)$ and $T:$ Spin $V^{\prime} \rightarrow S O\left(V^{\prime}\right)$ are surjective.

(c) The group $O(V)$ (resp. $\left.O\left(V^{\prime}\right)\right)$ acts transitively on $G r$ (resp. $\left.G r^{\prime}\right)$.

Proof. Let $U^{k}=\sum_{0 \neq|j| \leq k} \mathbb{C} v_{j}$ and $V^{k}=U^{k}+\mathbb{C} v_{0}$. It is well-known that the group of all orthogonal transformations of $U^{k}$ and $V^{k}$ is generated by reflections. This proves (a). (b) follows from (2.4) and the fact that $O\left(U^{k}\right)$ and $O\left(V^{k}\right)$ is generated by reflections. (c): If $W \in G r$ (resp. $G r^{\prime}$ ), then $W=W^{\prime}+U^{k}$ for $k>>0$, where $W^{\prime} \subset V^{k}$ (resp. $\left.\subset U^{k}\right)$. Now (c) follows from the well-known fact that $O\left(V^{k}\right)$ resp. $O\left(U^{k}\right)$ acts transitively on the set of maximal isotropic subspaces.

Corollary 2.1 The group $S O(V)$ acts transitively on $G r$. The group $S O\left(V^{\prime}\right)$ has two orbits on $G r^{\prime}$, that of $U_{0}$ and of $U_{0}^{\prime}=r_{v_{1}+v_{-1}}\left(U_{0}\right)$.

Corollary 2.2 Let $\tau \in F$ (resp. $\tau \in F_{\bar{\nu}}$ ). Then Ann $\tau$ is a maximal isotropic subspace in $V$ (resp. $\left.V^{\prime}\right)$ if and only if $\tau \in O$ (resp. $\tau \in O_{\bar{\nu}}$ ).

Proof. Suppose that Ann $\tau$ is maximal isotropic, then it lies in $G r$ (resp. $G r^{\prime}$ ). Using corollary 2.1, we may find a $g \in S O(V)$ (resp. $S O\left(V^{\prime}\right)$ ) such that $g A n n \tau=U_{0}$ (resp. $=U_{0}$ or $U_{0}^{\prime}$ ). Taking $\tilde{g} \in \operatorname{Spin} V$ (resp. $\in$ Spin $V^{\prime}$ ), a preimage of $g$, we see that Ann $\tilde{g} \tau=U_{0}$ (resp. $=U_{0}$ or $\left.U_{0}^{\prime}\right)$ by (2.9). It follows that, up to a constant, $\tilde{g} \tau=|0\rangle$ (resp. $|0\rangle$ or $|1\rangle$ ).

We can now prove Theorem 1.2 stated in the Introduction, where $S$ and $S^{\prime}$ are given by (2.7). 
Proof of Theorem 1.2. We only give the proof of (a). The proof of (b) is completely analogous.

Since $S$ commutes with the diagonal action of the group $\operatorname{Spin} V$ (see Lemma 2.1) and since the second equation of (2.8) holds for $\tau=|0\rangle$, any $\tau \in O$ satisfies (1.18).

For the converse, we introduce a gradation on $F$ by letting

$$
\operatorname{deg}|0\rangle=0 \quad \operatorname{deg} v_{i}=i
$$

Write $\tau=\sum_{k=1}^{N} c_{k} \tau_{k}$, where any $\tau_{k}$ is a simple vector

$$
\tau_{k}=v_{j_{1}} v_{j_{2}} \ldots v_{j_{p}}|0\rangle \quad j_{1}>j_{2}>\ldots>j_{p}>0 .
$$

We can of course reorder and rescale in such a way that $\tau_{1}$ will be of maximal degree and $c_{1}=1$. If among the $\tau_{k}$ with $k>1$ there exists a $\tau_{\ell}$ of the form

$$
\tau_{\ell}=v_{i} v_{j} \tau_{1} \quad \text { with } \quad i+j<0
$$

we can remove this term by replacing $\tau$ by $\left(1-c_{\ell} v_{i} v_{j}\right) \tau$, which again satisfies (1.18). Such $1-c v_{i} v_{j} \in$ Spin $V$ for any $c \in \mathbb{C}$ and $i+j \neq 0$. Repeating this procedure a finite number of times we obtain an element of the form $\tau_{1}+\varphi\left(\varphi=\sum_{k=2}^{N} c_{k} \tau_{k}\right)$ where none of the simple vectors $\tau_{k}$ appearing in $\varphi$ is equal for $\tau_{1}$, or is of the form (2.10). Since $\tau_{1}$ is a simple vector, which by Lemma 2.3 lies in $O, \tau_{1}$ must satisfy (1.18). Hence $\tau_{1}$ and $\varphi$ satisfy:

$$
\sum_{j \in \mathbb{Z}} v_{j} \varphi \otimes v_{-j} \tau_{1}+v_{j} \tau_{1} \otimes v_{-j} \varphi+v_{j} \varphi \otimes v_{-j} \varphi=\frac{1}{2}\left(\varphi \otimes \tau_{1}+\tau_{1} \otimes \varphi+\varphi \otimes \varphi\right) .
$$

If $\varphi$ is non-zero, then (since $\varphi$ does not contain a simple vector proportional to $\tau_{1}$ ) there exists a $j \neq 0$ such that $v_{j} \tau_{1} \otimes v_{-j} \varphi \neq 0$. Then $v_{j} \tau_{1}$ must be equal to either (1) $\lambda v_{-i} \tau_{\ell}$ for some $-i>j$, (2) $\lambda \tau_{\ell}$ for some $\ell>1$ or to (3) $\lambda \tau_{1}$, where for all 3 cases $\lambda \in \mathbb{C}^{\times}$. Clearly (3) is impossible and (1) and (2) are also not possible since $\tau_{\ell}$ is not of the form (2.10) (where $i=0$ for case (2)). Hence $\varphi=0$ and $\tau=\tau_{1}$ is a simple vector, which by Lemma 2.3 lies in $O$.

Equation (1.18) (resp. (1.19) is called the BKP (resp. DKP) hierarchy because the group $S O(V)$ (resp. $S O\left(V^{\prime}\right)$ ) is of $B$ (resp. $D$ ) type and because after bosonization this equation becomes a hierarchy of PDE's similar to the $n$-component KP hierarchy (cf. Introduction). Let, for $g \in \operatorname{Spin} V^{\prime}, \tau_{\nu}=g|\nu\rangle$ and $\tau_{\mu}=g|\mu\rangle$, where $\nu, \mu=0$ or 1 and $\mu \neq \nu$. Then by Lemma 2.2 and the third and fourth equation of (2.8), $\tau_{\nu}$ and $\tau_{\mu}$ satisfy the Modified DKP hierarchy (see 1.20)

$$
S^{\prime}\left(\tau_{\nu} \otimes \tau_{\mu}\right)=\tau_{\mu} \otimes \tau_{\nu} .
$$

It has the following geometrical interpretation.

Theorem 2.1 Elements $\tau_{\nu} \in O_{\bar{\nu}}, \tau_{\mu} \in O_{\bar{\mu}}, \mu \neq \nu$, satisfy the Modified DKP hierarchy if and only if the space

$$
\left(A n n \tau_{0}+A n n \tau_{1}\right) /\left(A n n \tau_{0} \cap A n n \tau_{1}\right)
$$

is 2-dimensional and the induced bilinear form on it is non-degenerate. 
Proof. Without loss of generality we may assume that $\nu=1$ and $\mu=0$ and that $\tau_{\mu}=|0\rangle$. Suppose first that (1.20) holds, then

$$
\sum_{j<0} v_{j} \tau_{1} \otimes v_{-j}|0\rangle=|0\rangle \otimes \tau_{1} .
$$

Since all $v_{-j}|0\rangle$ for $j<0$ are linearly independent, we conclude that $\left(\sum_{j<0} a_{j} v_{j}\right) \tau_{1}=|0\rangle$. Hence there exists an isotropic vector $w_{0} \in V^{\prime}$ such that $|0\rangle=w_{0} \tau_{1}$. Now notice that $w_{0}|0\rangle=w_{0}^{2} \tau_{1}=\frac{1}{2}\left(w_{0}, w_{0}\right) \tau_{1}=0$. Because of the symmetry of the equation (1.20), there also exists an isotropic vector $w_{1} \in V^{\prime}$ such that $\tau_{1}=w_{1}|0\rangle$. Hence

$$
|0\rangle=w_{0} w_{1}|0\rangle=\left(\left(w_{0}, w_{1}\right)-w_{1} w_{0}\right)|0\rangle=\left(w_{0}, w_{1}\right)|0\rangle
$$

and therefore $\left(w_{0}, w_{1}\right)=1$. This leads to the statement of the theorem about the annihilator spaces of $\tau_{0}$ and $\tau_{1}$.

Conversely,assume that $\tau_{1}=w_{1} \tau_{0}$ for some isotropic vector $w_{1} \in V^{\prime}$. Then

$$
\begin{aligned}
\sum_{0 \neq i \in \mathbb{Z}} v_{i} \tau_{1} \otimes v_{-i} \tau_{0} & =\sum_{0 \neq i \in \mathbb{Z}} v_{i} w_{1} \tau_{0} \otimes v_{-i} \tau_{0} \\
& =\sum_{0 \neq i \in \mathbb{Z}}\left(v_{i}, w_{1}\right)-w_{1} v_{i} \tau_{0} \otimes v_{-i} \tau_{0} \\
& =\tau_{0} \otimes w_{1} \tau_{0}-\left(w_{1} \otimes 1\right) S^{\prime}\left(\tau_{0} \otimes \tau_{0}\right) \\
& =\tau_{0} \otimes \tau_{1},
\end{aligned}
$$

since $\tau_{0}$ satisfies (1.19).

Let $\tau \in O$, we split up $\tau$ as $\tau=\tau_{0}+\tau_{1}$, where $\tau_{\nu} \in F_{\bar{\nu}}$ (recall that $F_{\bar{\nu}}$ are eigenspaces for $\left.-\sqrt{2} v_{0}\right)$. We now rewrite (1.18):

$$
S^{\prime}\left(\left(\tau_{0}+\tau_{1}\right) \otimes\left(\tau_{0}+\tau_{1}\right)\right)+v_{0}\left(\tau_{0}+\tau_{1}\right) \otimes v_{0}\left(\tau_{0}+\tau_{1}\right)=\frac{1}{2}\left(\tau_{0}+\tau_{1}\right) \otimes\left(\tau_{0}+\tau_{1}\right) .
$$

The left-hand side of this equation is equal to

$$
S^{\prime}\left(\left(\tau_{0}+\tau_{1}\right) \otimes\left(\tau_{0}+\tau_{1}\right)\right)+\frac{1}{2}\left(\tau_{0}-\tau_{1}\right) \otimes\left(\tau_{0}-\tau_{1}\right) .
$$

Hence, (2.11) splits up into 4 separate equations:

$$
\begin{aligned}
& S^{\prime}\left(\tau_{\nu} \otimes \tau_{\nu}\right)=0 \quad(\nu=0 \text { or } 1), \\
& S^{\prime}\left(\tau_{0} \otimes \tau_{1}\right)=\tau_{1} \otimes \tau_{0}, \\
& S^{\prime}\left(\tau_{1} \otimes \tau_{0}\right)=\tau_{0} \otimes \tau_{1},
\end{aligned}
$$

which are exactly the equations of the DKP and Modified DKP-hierarchy (1.19) and (1.20). Hence we have proved Theorem 1.3, stated in the Introduction (where $S$ and $S^{\prime}$ are given by $(2.7)$ ).

So we conclude that the fermionic BKP hierarchy contains the DKP hierarchy and the modified DKP hierarchy. We will now show that it also contains the KP hierarchy and modified KP hierarchy as well. We split up in some way the maximal isotropic subspace $U_{0}$ into two infinite dimensional subspaces $U_{0}^{ \pm}$:

$$
U_{0}=U_{0}^{+} \oplus U_{0}^{-} .
$$


Choose bases $\left\{v_{-i}^{ \pm}\right\}_{i \in \mathbb{Z}_{+}+\frac{1}{2}}$ of $U_{0}^{ \pm}$and include these bases in a basis of $V$ by adding vectors $\left\{v_{i}^{\mp}\right\}_{i \in \mathbb{Z}_{+}+\frac{1}{2}}$ and $v_{0}$ such that

$$
\left(v_{i}^{ \pm}, v_{j}^{ \pm}\right)=0,\left(v_{i}^{ \pm}, v_{j}^{\mp}\right)=\delta_{i,-j},\left(v_{i}^{ \pm}, v_{0}\right)=0,\left(v_{0}, v_{0}\right)=1 .
$$

We will assume from now on that we have chosen these bases in such a way that

$$
v_{\frac{1}{2}}^{+}|0\rangle=v_{1}|0\rangle
$$

The Grassmannian $G r$ (resp. $G r^{\prime}$ ) translates in this picture to the set of all maximal isotropic subspaces $W \subset V$ (resp. $\subset V^{\prime}$ ) such that $U_{k} \subset W$ for $k>>0$, where $U_{k}=$ $U_{k}^{+} \oplus U_{k}^{-}$and $U_{k}^{ \pm}=\sum_{j<-k} \mathbb{C} v_{j}^{ \pm}$.

Define

$$
V^{ \pm}=\sum_{j \in \mathbb{Z}+\frac{1}{2}} \mathbb{C} v_{j}^{ \pm},
$$

so that $V=V^{+}+\mathbb{C} v_{0}+V^{-}$. Consider the subgroup $\overline{\operatorname{Spin} V}$ of Spin $V$ generated by all elements of the form $1+a v_{i}^{+} v_{j}^{-}, a \in \mathbb{C}$. It preserves each of the subspaces $V^{+}$and $V^{-}$ and we have the induced isomorphisms $\overline{\operatorname{Spin} V} \sim \vec{\rightarrow} G L$, where $G L$ is the subgroup of $S O(V)$, consisting of all elements $g \in S O(V)$ such that $g\left(V^{ \pm}\right)=V^{ \pm}$.

Lemma 2.4 Let $g \in G L$ be such that

$$
g v_{j}^{+}=\sum_{i \in \mathbb{Z}+\frac{1}{2}} A_{i j} v_{i}^{+}
$$

and let $A=\left(A_{i j}\right)_{i, j \in \mathbb{Z}+\frac{1}{2}}$ and $A^{-1}=\left(B_{i j}\right)_{i, j \in \mathbb{Z}+\frac{1}{2}}$. Then

$$
g v_{j}^{-}=\sum_{i \in \mathbb{Z}+\frac{1}{2}} B_{-j i} v_{-i}^{-}
$$

and

$$
g v_{0}=v_{0}
$$

Proof. Suppose that $g v_{\ell}^{-}=\sum_{k} B_{-\ell, k} v_{-k}^{-}$, then it follows from

$$
\begin{aligned}
\delta_{j,-\ell}=\left(v_{j}^{+}, v_{\ell}^{-}\right) & =\left(\sum_{i} A_{i j} v_{i}^{+}, B_{-\ell k} v_{-k}^{-}\right) \\
& =\sum_{i} A_{i j} B_{-\ell, i}
\end{aligned}
$$

that $B=A^{-1}$. Since $g \in S O(V), g v_{0}=v_{0}$.

In order to describe the group orbits of $\overline{\operatorname{Spin} V}$, we introduce the restricted Grassmannian

$$
\overline{G r}=\bigcup_{\ell \in \mathbb{Z}} \overline{G r}^{(\ell)},
$$

where $\overline{G r}^{(\ell)}$ is the set of all maximal isotropic subspaces $W \subset V^{\prime} \subset V$ such that $W=$ $W^{+} \oplus W^{-}$with $U_{k}^{ \pm} \subset W^{ \pm} \subset V^{ \pm}$for $k>>0$ and $\operatorname{dim}\left(W^{+} / U_{k}^{+}\right)-\operatorname{dim}\left(W^{-} / U_{k}^{-}\right)=2 \ell$. We will call $\ell$ the charge of $\overline{G r}^{(\ell)}$. Notice that, since $W$ is maximal isotropic,

$$
W^{-}=\left(W^{+}\right)^{\perp} \cap V^{-} .
$$


Hence $W^{+}$determines $W$.

We decompose the space $F$ into charge sectors as follows. Define

$$
\text { charge }|0\rangle=0, \quad \text { charge } v_{j}^{ \pm}= \pm 1, \quad \text { charge } v_{0}=0,
$$

and let

$$
F=\bigoplus_{k \in \mathbb{Z}} F_{k}, \text { where } F_{k}=\{f \in F \mid \text { charge } f=k\} .
$$

Since charge $\left(1+a v_{i}^{+} v_{j}^{-}\right)=0, \overline{\text { Spin } V}$ leaves all spaces $F_{k}$ invariant.

Define the following vectors in $F_{k}$ :

$$
|k\rangle= \begin{cases}v_{k-\frac{1}{2}}^{+} v_{k-\frac{3}{2}}^{+} \cdots v_{\frac{1}{2}}^{+}|0\rangle & \text { for } \quad k \geq 0 \\ v_{-k-\frac{1}{2}}^{-} v_{-k-\frac{3}{2}}^{-} \cdots v_{\frac{1}{2}}^{-}|0\rangle & \text { for } \quad k<0\end{cases}
$$

One has

$$
\text { Ann } \mid k>=\sum_{j<k} \mathbb{C} v_{j}^{+}+\sum_{j>k} \mathbb{C} v_{-j}^{-}
$$

and clearly Ann $|k\rangle \in \overline{G r}^{(k)}$.

Lemma 2.5 The group $G L$ acts transitively on $\overline{G r}^{(\ell)}$ for any $\ell \in \mathbb{Z}$.

Proof. Let $W \in \overline{G r}^{(\ell)}$, then by (2.13) $W=W^{+} \oplus\left(\left(W^{+}\right)^{\perp} \cap V^{-}\right)$, hence $W^{+}$determines $W$. Now choose a basis $w_{\ell-\frac{1}{2}}, w_{\ell-\frac{3}{2}}, \ldots$ of $W^{+}$in such a way that $w_{\ell-p}=v_{\ell-p}^{+}$for $p>>0$. Clearly there exists an invertible matrix $A=\left(A_{i j}\right)_{i, j \in \mathbb{Z}_{\frac{1}{2}}}$ such that $w_{j}=\sum_{i} A_{i j} v_{i}^{+}$. Hence by lemma (2.4), this $A$ determines a unique $g \in G L$ such that $g\left(\sum_{j<\ell} \mathbb{C} v_{j}^{+}\right)=W^{+}$. Thus $g($ Ann $|\ell\rangle)=W$.

We express the operator $S$ in terms of the new basis

$$
S=v_{0} \otimes v_{0}+\sum_{j \in \mathbb{Z}+\frac{1}{2}} v_{j}^{+} \otimes v_{-j}^{-}+v_{j}^{-} \otimes v_{-j}^{+} .
$$

We split this operator as follows

$$
\begin{aligned}
& S=S^{0}+S^{+}+S^{-} \text {with } \\
& S^{0}=v_{0} \otimes v_{0} \text { and } S^{ \pm}=\sum_{j \in \mathbb{Z}+\frac{1}{2}} v_{j}^{ \pm} \otimes v_{-j}^{\mp} .
\end{aligned}
$$

It is straightforward to check that for $k, \ell \in \mathbb{Z}$, with $\ell \geq 0$, one has

$$
\begin{array}{ll}
S^{0}(|k+\ell\rangle \otimes|k\rangle) & =\frac{(-)^{\ell}}{2}|k+\ell\rangle \otimes|k\rangle, \\
S^{+}(|k+\ell\rangle \otimes|k\rangle) & =S^{-}(|k\rangle \otimes|k+\ell\rangle)=0, \\
\left(S^{+}\right)^{\ell}(|k\rangle \otimes|k+\ell\rangle) & =(-)^{\frac{\ell(\ell-1)}{2}} \ell !|k+\ell\rangle \otimes|k\rangle, \\
\left(S^{-}\right)^{\ell}(|k+\ell\rangle \otimes|k\rangle) & =(-)^{\frac{\ell(\ell-1)}{2}} \ell !|k\rangle \otimes|k+\ell\rangle, \\
\left(S^{+}\right)^{\ell+1}(|k\rangle \otimes|k+\ell\rangle) & =\left(S^{-}\right)^{\ell+1}(|k+\ell\rangle \otimes|k\rangle)=0 .
\end{array}
$$


By Lemma 2.1, $S$ commutes with the diagonal action of Pin $V$. Since for any $g \in$ $\overline{\text { Spin } V}, T_{g}\left(V^{ \pm}\right)=V^{ \pm}$and $T_{g}\left(v_{0}\right)=v_{0}$, we deduce that $S^{0}, S^{+}$and $S^{-}$separately commute with the diagonal action of $\overline{\operatorname{Spin} V}$. Denote by $\overline{O_{k}}$ the Spin $V$ group orbit of $|k\rangle$. Let $g \in \overline{\operatorname{Spin} V}$ and let $\tau_{k}=g|k\rangle$ for all $k \in \mathbb{Z}$, then since (2.15) holds one has for $k, \ell \in \mathbb{Z} \quad \ell \geq 0$ :

$$
\begin{array}{ll}
S^{0}\left(\tau_{k+\ell} \otimes \tau_{k}\right) & =\frac{(-)^{\ell}}{2} \tau_{k+\ell} \otimes \tau_{k}, \\
S^{+}\left(\tau_{k+\ell} \otimes \tau_{k}\right) & =S^{-}\left(\tau_{k} \otimes \tau_{k+\ell}\right)=0, \\
\left(S^{+}\right)^{\ell}\left(\tau_{k} \otimes \tau_{k+\ell}\right) & =(-)^{\frac{\ell(\ell-1)}{2}} \ell !\left(\tau_{k+\ell} \otimes \tau_{k}\right), \\
\left(S^{-}\right)^{\ell}\left(\tau_{k+\ell} \otimes \tau_{k}\right) & =(-)^{\frac{\ell(\ell-1)}{2}} \ell ! \tau_{k} \otimes \tau_{k+\ell}, \\
\left(S^{+}\right)^{\ell+1}\left(\tau_{k} \otimes \tau_{k+\ell}\right) & =\left(S^{-}\right)^{\ell+1}\left(\tau_{k+\ell} \otimes \tau_{k}\right)=0 .
\end{array}
$$

The second equation of (2.16) is called the $\ell$-th modified KP hierarchy; the 0-th modified $\mathrm{KP}$ hierarchy is also known as the KP hierarchy.

Theorem 2.2 Let $\tau_{k} \in F_{k}, k \in \mathbb{Z}$. Then $\tau_{k} \in \overline{O_{k}}$ if and only if $\tau_{k}$ satisfies the $K P$ hierarchy, i.e., satisfies the second equation of (2.10) for $\ell=0$.

The proof of this Theorem is similar to the proof of theorem 1.2. For a proof see [10].

Theorem 2.3 Let $k \in \mathbb{Z}, \ell \in \mathbb{Z}_{+}, \tau_{k} \in \overline{O_{k}}$ and $\tau_{k+\ell} \in \overline{O_{k+\ell}}$, then one has the following 6 equivalent formulations of the $\ell$-th modified KP hierarchy.

(a) $S^{+}\left(\tau_{k+\ell} \otimes \tau_{k}\right)=0$,

(b) $S^{-}\left(\tau_{k} \otimes \tau_{k+\ell}\right)=0$,

(c) $\left(S^{+}\right)^{\ell}\left(\tau_{k} \otimes \tau_{k+\ell}\right)=(-)^{\frac{\ell(\ell-1)}{2}} \ell ! \tau_{k+\ell} \otimes \tau_{k}$,

(d) $\left(S^{-}\right)^{\ell}\left(\tau_{k+\ell} \otimes \tau_{k}\right)=(-)^{\frac{\ell(\ell-1)}{2}} \ell ! \tau_{k} \otimes \tau_{k+\ell}$,

(e) $A n n \tau_{k} \cap V^{+} \subset A n n \tau_{k+\ell} \cap V^{+}$,

(f) Ann $\tau_{k+\ell} \cap V^{-} \subset$ Ann $\tau_{k} \cap V^{-}$.

Proof. The equivalences (a) $\Leftrightarrow$ (b), (c) $\Leftrightarrow$ (d) and (e) $\Leftrightarrow$ (f) are obvious. Without loss of generality we may assume from now on in this proof that $\tau_{k}=|k\rangle$.

First assume (a) holds; since $\tau_{k}=|k\rangle$ one has

$$
\sum_{i<k} v_{i}^{+} \tau_{k+\ell} \otimes v_{-i}^{-}|k\rangle=0
$$

Hence, $v_{i}^{+} \tau_{k+\ell}=0$ for all $i<k$, so (e) holds:

$$
\text { Ann }|k\rangle \cap V^{+} \subset \text { Ann } \tau_{k+\ell} \cap V^{+} .
$$

Now suppose (e) holds, then

$$
\sum_{i \in \frac{1}{2}+\mathbb{Z}} v_{i}^{+} \tau_{k+\ell} \otimes|k\rangle=\sum_{i<k} v_{i}^{+} \tau_{k+\ell} \otimes v_{-i}^{-}|k\rangle,
$$


but this is equal to 0 since all $v_{i}^{+} \in A n n|k\rangle$ for $i<k$ and by (e) also $v_{i}^{+} \in A n n \tau_{k+\ell}$ for $i<k$. This proves the equivalence (a) $\Leftrightarrow(\mathrm{e})$.

Next assume that (c) holds, which is equivalent to

$$
\sum_{i_{1}>i_{2}>\ldots>i_{\ell}>k} v_{i_{1}}^{+} \ldots v_{i_{\ell}}^{+}|k\rangle \otimes v_{-i_{1}}^{-} \ldots v_{-i_{\ell}}^{-} \tau_{k+\ell}=(-)^{\frac{\ell(\ell-1)}{2}} \tau_{k+\ell} \otimes|k\rangle
$$

Since all $v_{i_{1}}^{+} \ldots v_{i_{\ell}}^{+}|k\rangle$ are linearly independent we find that

$$
\tau_{k+\ell}=\sum_{i_{1}>i_{2}>\ldots i_{\ell}>k} \mu_{i_{1}, \ldots i_{\ell}} v_{i_{1}}^{+} v_{i_{2}}^{+} \ldots v_{i_{\ell}}^{+}|k\rangle
$$

From which one deduces that

$$
\text { Ann }|k\rangle \cap V^{+} \subset \text { Ann } \tau_{k+\ell} \cap V^{+} \text {. }
$$

Hence (c) implies (e).

Now assume that (e) holds; one has

$$
\text { Ann } \tau_{k+\ell} \cap V^{+}=\left(A n n|k\rangle \cap V^{+}\right) \cup<w_{1}^{+}, w_{2}^{+}, \ldots, w_{\ell}^{+}>
$$

for certain $w_{i}^{+} \in V^{+} w_{i}^{+} \notin A n n|k\rangle, 1 \leq i \leq \ell$.

Without loss of generality we may assume that $\tau_{k+\ell}=w_{1}^{+} w_{2}^{+} \ldots w_{\ell}^{+}|k\rangle$, so

$$
\begin{aligned}
& \left(S^{+}\right)^{\ell}\left(|k\rangle \otimes \tau_{k+\ell}\right)=\sum_{i_{1}, \ldots, i_{\ell} \in \mathbb{Z}+\frac{1}{2}} v_{i_{1}}^{+} \ldots v_{i_{\ell}}^{+}|k\rangle \otimes v_{i_{1}}^{-} \ldots v_{-i_{\ell}}^{-} w_{1}^{+} \ldots w_{\ell}^{+}|k\rangle \\
& =\sum_{i_{1}, \ldots, i_{\ell} \in \mathbb{Z}+\frac{1}{2}} \sum_{\sigma \in S_{\ell}}(-)^{\frac{\ell(\ell-1)}{2}}(-)^{\operatorname{sign}(\sigma)}\left(v_{-i_{1}}^{-}, w_{\sigma\left(i_{1}\right)}^{+}\right) \times \\
& \left(v_{-i_{2}}^{-}, w_{\sigma\left(i_{2}\right)}^{+}\right) \ldots\left(v_{-i_{\ell}}^{-}, w_{\sigma\left(i_{\ell}\right)}^{+}\right) v_{i_{1}}^{+} v_{i_{2}}^{+} \ldots v_{i_{\ell}}^{+}|k\rangle \otimes|k\rangle \\
& =(-)^{\frac{\ell(\ell-1)}{2}} \ell ! w_{1}^{+} w_{2}^{+} \ldots w_{\ell}^{+}|k\rangle \otimes|k\rangle \\
& =(-)^{\frac{\ell(\ell-1)}{2}} \ell ! \tau_{k+\ell} \otimes|k\rangle \text {. }
\end{aligned}
$$

Hence (e) implies (c).

Since $|1\rangle=v_{1}|0\rangle=v_{\frac{1}{2}}^{+}|0\rangle$, we find that $|k\rangle \in F_{\bar{k}}$ (here, as before, $\bar{k}=k \bmod 2$ ). Now Ann $|k\rangle$ is an element of $G r$ and $G r^{\prime}$ hence $|k\rangle \in O$ and $|k\rangle \in O_{\bar{k}}$. Therefore one has the following

Proposition 2.1 Let $\tau_{k} \in F_{k}, \tau_{k+1} \in F_{k+1}$ (so that $\tau_{k} \in F_{\bar{k}}$ and $\tau_{k+1} \in F_{\overline{k+1}}$ ). Then:

(a) $\tau_{k}$ satisfies the KP hierarchy if and only if it satisfies the DKP hierarchy.

(b) $\tau_{k}$ and $\tau_{k+1}$ satisfy the first modified KP hierarchy if and only if they satisfy the modified DKP hierarchy.

(c) $\tau_{k}$ and $\tau_{k+1}$ satisfy the KP hierarchy and the first modified KP hierarchy, if and only if $\tau_{k}+\tau_{k+1}$ satisfies the BKP hierarchy.

The proof is straightforward. 


\section{Orthogonal multicomponent boson-fermion correspondence}

Using a bosonization one can rewrite (1.18), (1.20) and (2.16) as a system of partial differential equations. There are however many different bosonizations. In this paper we will consider several possibilities. The simplest one is given in the introduction.

\section{$3.1 n$-component charged fermions and $D_{n}^{(1)}$}

In this section we will explain the $n$-component generalization of the classical bosonfermion correspondence, which was described in detail in the Introduction.

Choose bases

$\left\{\psi_{i}^{a}\right\}_{i \in \mathbb{Z}+\frac{1}{2}}$, where $a=1,2, \ldots, n$, of $V^{ \pm}$such that $\psi_{i}^{ \pm a} \in U_{0}$ for $i>0$ and

$$
\left(\psi_{i}^{a}, \psi_{j}^{b}\right)=\delta_{a,-b} \delta_{i,-j} .
$$

Define the fermionic fields $(a \in \mathbb{Z}, 0<|a| \leq n)$ :

$$
\psi^{a}(z)=\sum_{i \in \frac{1}{2}+\mathbb{Z}} \psi_{i}^{a} z^{-i-\frac{1}{2}}
$$

Then one has the following commutation relations:

$$
\left[\psi^{a}(y), \psi^{b}(z)\right]_{+}=\delta_{a,-b} \delta(y-z) .
$$

We let

$$
e^{a b}(z)=\sum_{m \in \mathbb{Z}} e_{m}^{a b} z^{-m-1}=: \psi^{a}(z) \psi^{b}(z):
$$

where the normal ordering is defined as in the introduction.

It is immedate that

$$
e_{m}^{a b}=-e_{m}^{b a} \quad \text { hence } \quad e_{m}^{a a}=0 .
$$

Using Wick's formula, it is straightforward to check that the operators $e_{m}^{a b}$ form a representation in $F$ of the affine Kac-Moody algbera $D_{n}^{(1)}$ of level 1 [7]:

$$
\begin{aligned}
{\left[e^{a b}(y), e^{c d}(z)\right] } & =\left(\delta_{b,-c} e^{a d}(z)+\delta_{b,-d} e^{c d}(z)-\delta_{a,-c} e^{b d}(z)-\delta_{a,-d} e^{c b}(z)\right) \delta(y-z) \\
& +\left(\delta_{a,-d} \delta_{b,-c}-\delta_{a,-c} \delta_{b,-d}\right) \delta_{z}^{\prime}(y-z) .
\end{aligned}
$$

The Lie algebra $D_{n}^{(1)}$ acts on the Clifford algebra via the adjoint representation

$$
\left[e^{a b}(y), \psi^{c}(z)\right]=\left(\delta_{b,-c} \psi^{a}(z)-\delta_{a,-c} \psi^{b}(z)\right) \delta(y-z)
$$

Let $\alpha^{a}(z)=e^{a,-a}(z)$, then we have :

$$
\begin{aligned}
{\left[\psi^{a}(y), \alpha^{b}(z)\right] } & =-s(a) \delta_{|a|, b} \psi^{a}(z) \delta(y-z), \\
{\left[\alpha^{a}(y), \alpha^{b}(z)\right] } & =\delta_{a, b} \delta_{z}^{\prime}(y-z),
\end{aligned}
$$

in particular, the $\alpha_{m}^{a}$ form an oscillator algebra. Here and further we let $s(a)=a /|a|$. 
The charge operator $\alpha_{0}$ of the previous section is now $\alpha_{0}=\sum_{a=1}^{n} \alpha_{0}^{a}$. Again it is possible to express the fermionic fields in terms of the oscillator algebra. However in the $n$-component case we need $n$ additional operators $Q_{a}(a=1,2, \ldots, n)$ on $F$ defined (uniquely) by

$$
\begin{aligned}
Q_{a}|0\rangle & =\psi_{-\frac{1}{2}}^{a}|0\rangle, \\
Q_{a} \psi_{k}^{b} & =(-)^{1-\delta_{a,|b|}} \psi_{k-s(b) \delta_{a,|b|}}^{b} Q_{a} .
\end{aligned}
$$

They satisfy the following commutation relations

$$
\begin{aligned}
Q_{a} Q_{b} & =-Q_{b} Q_{a} \text { if } a \neq b, \\
{\left[\alpha_{k}^{a}, Q_{b}\right] } & =\delta_{a b} \delta_{k, 0} Q_{b}, \\
Q_{a} v_{0} & =-v_{0} Q_{a} .
\end{aligned}
$$

As in the 1-component case one has

Theorem 3.1 ( 17$]$ )

$$
\psi^{a}(z)=Q_{a}^{s(a)} z^{s(a) \alpha_{0}^{a}} \exp \left(-s(a) \sum_{k<0} \frac{\alpha_{k}^{a}}{k} z^{-k}\right) \exp \left(-s(a) \sum_{k>0} \frac{\alpha_{k}^{a}}{k} z^{-k}\right) .
$$

We can now describe the $n$-component charged (or untwisted) boson-fermion correspondence. Let $\mathfrak{L}$ be the root lattice of type $B_{n}$, i.e., $\mathfrak{L}$ is a lattice with basis $\delta_{a}(1 \leq q \leq n)$ over $\mathbb{Z}$ and symmetric bilinear form $\left(\delta_{i} \mid \delta_{j}\right)=\delta_{i j}$. Let

$$
\epsilon_{i j}= \begin{cases}-1 & \text { if } \quad i>j \\ 1 & \text { if } \quad i \leq j\end{cases}
$$

Define a bimultiplicative function $\epsilon: \mathfrak{L} \times \mathfrak{L} \rightarrow\{ \pm 1\}$ by letting

$$
\epsilon\left(\delta_{i}, \delta_{j}\right)=\epsilon_{i j}
$$

Let $\delta=\delta_{1}+\delta_{2}+\ldots+\delta_{n}$ and set $\mathfrak{M}=\{\gamma \in \mathfrak{L} \mid(\delta \mid \gamma)=0\}$ and $\mathfrak{N}=\{\gamma \in \mathfrak{L} \mid(\delta \mid \gamma) \in 2 \mathbb{Z}\}$. Then $\mathfrak{M}($ resp. $\mathfrak{N})$ is the root lattice of $A_{n-1}$ (resp. $D_{n}$ ). Consider the vector space $\mathbb{C}_{\epsilon}[\mathfrak{L}]$ with basis $e^{\gamma}, \gamma \in \mathfrak{L}$, and the following twisted group algebra product

$$
e^{\alpha} e^{\beta}=\epsilon(\alpha, \beta) e^{\alpha+\beta}
$$

In the 1-component case $q=e^{\delta_{1}}$. Let $\mathbb{C}[x]$ be the space of polynomials in the indeterminates $t=\left\{t_{k}^{(a)}\right\}, k=1,2, \ldots, a=\frac{1}{2}, \frac{3}{2}, \ldots, n-\frac{1}{2}$. Define $B=\mathbb{C}[t] \otimes_{\mathbb{C}} \mathbb{C}_{\epsilon}[\mathfrak{L}]$, the tensor product of algebras. Then the $n$-component boson-fermion correspondence is the vector space isomorphism

$$
\sigma: F \stackrel{\sim}{\rightarrow} B
$$

given by

$$
\begin{aligned}
& \sigma\left(\alpha_{-m_{1}}^{a_{1}} \alpha_{-m_{2}}^{a_{2}} \ldots \alpha_{-m_{s}}^{a_{s}} Q_{\frac{1}{2}}^{k_{\frac{1}{2}}} Q_{\frac{3}{2}}^{k_{\frac{3}{2}}} \ldots Q_{n-\frac{1}{2}}^{k_{n-\frac{1}{2}}}|0\rangle\right)= \\
& m_{1} m_{2} \ldots m_{s} t_{m_{1}}^{\left(a_{1}\right)} t_{m_{2}}^{\left(a_{2}\right)} \ldots t_{m_{s}}^{\left(a_{s}\right)} \otimes e^{k_{\frac{1}{2}} \delta_{\frac{1}{2}}+k_{\frac{3}{2}} \delta_{\frac{3}{2}}+\ldots+k_{n-\frac{1}{2}} \delta_{n-\frac{1}{2}}}
\end{aligned}
$$


The transported charge is then as follows

$$
\text { charge }\left(p(t) \otimes e^{\gamma}\right)=(\delta \mid \gamma) \text {. }
$$

We denote the transported charge decomposition by

$$
B=\bigoplus_{m \in \mathbb{Z}} B_{m}
$$

Notice that one has the following isomorphism

$$
\sigma\left(F_{\bar{\nu}}\right)=\bigoplus_{m \in \nu+2 \mathbb{Z}} B_{m}
$$

The transported action of the operators is as follows

$$
\begin{aligned}
\sigma \alpha_{-m}^{a} \sigma^{-1}\left(p(t) \otimes e^{\gamma}\right) & =m t_{m}^{(a)} p(t) \otimes e^{\gamma}, \\
\sigma \alpha_{m}^{a} \sigma^{-1}\left(p(t) \otimes e^{\gamma}\right) & =\frac{\partial p(t)}{\partial t_{m}^{(a)}} \otimes e^{\gamma}, \\
\sigma \alpha_{0}^{a} \sigma^{-1}\left(p(t) \otimes e^{\gamma}\right) & =\left(\delta_{a} \mid \gamma\right) p(t) \otimes e^{\gamma}, \\
\sigma Q_{a} \sigma^{-1}\left(p(t) \otimes e^{\gamma}\right) & =\epsilon\left(\delta_{a}, \gamma\right) p(t) \otimes e^{\gamma+\delta_{a}},
\end{aligned}
$$

so $\sigma Q_{a} \sigma^{-1}=e^{s(a) \delta_{a}}$. Introduce for $\alpha \in \mathfrak{L}$ the operator $Z^{\alpha}$ on $B$ :

$$
z^{\alpha}\left(\left(p(t) \otimes e^{\gamma}\right)=z^{(\alpha \mid \gamma)} p(t) \otimes e^{\gamma} .\right.
$$

Then

$$
\sigma \psi^{a}(z) \sigma^{-1}=e^{s(a) \delta_{a}} z^{s(a) \delta_{a}} e^{s(a) \xi_{a}(t, z)} e^{-s(a) \eta_{a}(t, z)},
$$

where

$$
\xi_{a}(t, x)=\sum_{i=1}^{\infty} t_{i}^{(a)} z^{i} \quad \text { and } \quad \eta_{a}(t, z)=\sum_{i=1}^{\infty} \frac{1}{i} \frac{\partial}{\partial t_{i}^{(a)}} z^{-i}
$$

\subsection{1-component neutral fermions}

Here we recall the classical neutral boson-fermion correspondence. Let Let $\left\{\psi_{i}\right\}_{i \in \mathbb{Z}}$ be a basis of $V$ such that

$$
\left(\psi_{i}, \psi_{j}\right)=(-)^{i} \delta_{i,-j}
$$

and $\psi_{i}$ with $i>0$ lie in $U_{0}$. Define the generating series

$$
\psi(z)=\sum_{j \in \mathbb{Z}} \psi_{j} z^{-j}
$$

Then we have:

$$
[\psi(y), \psi(-z)]_{+}=z \delta(y+z) .
$$

Let $\psi(z)_{+}=\sum_{i=0}^{\infty} \psi_{-i} z^{i}$ and $\psi(z)_{-}=\psi(z)-\psi(z)_{+}$. Define

$$
\alpha(z)=\sum_{k \in 2 \mathbb{Z}+1} \alpha_{k} z^{-k-1}=: \psi(z) \frac{\psi(-z)}{z}:=\psi(z)_{+} \frac{\psi(-z)}{z}-\frac{\psi(-z)}{z} \psi(z)_{-} .
$$


Then it is straightforward to check (using Wick's formula) that:

$$
\begin{aligned}
& {[\psi(y), \alpha(z)]=\psi(-z) \delta(y+z)-\psi(z) \delta(y-z),} \\
& {[\alpha(y), \alpha(z)]=\delta_{z}^{\prime}(y-z)-\delta_{z}^{\prime}(y+z)}
\end{aligned}
$$

which means that $(m \in 2 \mathbb{Z}+1, n \in \mathbb{Z})$ :

$$
\begin{aligned}
& {\left[\alpha_{m}, \psi_{n}\right]=2 \psi_{n-m},} \\
& {\left[\alpha_{m}, \alpha_{n}\right]=2 m \delta_{m,-n} .}
\end{aligned}
$$

The $\alpha_{m}$ form a twisted oscillator algebra. There is no charge operator $\alpha_{0}$, therefore the elements $\psi_{i}$ from the Clifford algebra are called neutral fermions. In this case it is possible to express the fermions completely in terms of the oscillator algebra.

Theorem 3.2 ( [4])

$$
\psi(z)=-\frac{1}{\sqrt{2}} \exp \left(-\sum_{k<0, \text { odd }} \frac{\alpha_{k}}{k} z^{-k}\right) \exp \left(-\sum_{k>0, \text { odd }} \frac{\alpha_{k}}{k} z^{-k}\right) .
$$

The neutral or twisted boson-fermion correspondece consists of identifying the space $F$ with the space $B=\mathbb{C}\left[t_{1}, t_{3}, t_{5}, \ldots\right]$ via the vector space isomorphism $\sigma: F \rightarrow B$ given by

$$
\sigma\left(\alpha_{-m_{1}} \alpha_{-m_{2}} \ldots \alpha_{-m_{s}}|0\rangle\right)=m_{1} m_{2} \ldots m_{s} t_{m_{1}} t_{m_{2}} \ldots t_{m_{s}} .
$$

The transported action of the operators $\alpha_{m}$ is as follows

$$
\begin{aligned}
\sigma \alpha_{-m} \sigma^{-1}(p(t)) & =m t_{m} p(t), \\
\sigma \alpha_{m} \sigma^{-1}(p(t)) & =2 \frac{\partial p(t)}{\partial t_{m}} .
\end{aligned}
$$

Then

$$
\begin{gathered}
\sigma \psi(z) \sigma^{-1}=-\frac{1}{2} \sqrt{2} e^{\xi(t, z)} e^{-\eta(t, z)} \quad \text { where } \\
\xi(t, z)=\sum_{i=0}^{\infty} t_{2 i+1} z^{2 i+1} \quad \eta(t, z)=\sum_{i=0}^{\infty} \frac{2}{2 i+1} \frac{\partial}{\partial t_{2 i+1}} z^{-2 i-1} .
\end{gathered}
$$

\section{$3.3 n$-component neutral fermions}

In the neutral (or twisted) case we make a small distinction between $n$ even and $n$ odd. So let $n=2 m+1$ if $n$ is odd and $n=2 m$ if $n$ is even. We choose the following basis of $V$.

$$
\begin{array}{ll}
\left\{\psi_{i}^{a}\right\}_{i \in \mathbb{Z}, a=0, \pm 1, \pm 2, \ldots \pm m} & \text { if } n \text { is odd, } \\
\left\{\psi_{i}^{a}\right\}_{i \in \mathbb{Z}, a= \pm 1, \pm 2, \ldots \pm m} \cup\left\{v_{0}\right\} & \text { if } n \text { is even, }
\end{array}
$$

such that they satisfy the following conditions

$$
\begin{array}{ll}
\left(\psi_{i}^{a}, \psi_{j}^{b}\right)=(-)^{i} \delta_{a, b} \delta_{i,-j}, & \\
\left(\psi_{i}^{a}, v_{0}\right)=0 & \text { if } n \text { is even, } \\
\psi_{i}^{a} \in U_{0} & \text { if } i>0 .
\end{array}
$$


Then

$$
\left[\psi^{a}(y), \psi^{b}(-z)\right]_{+}=\delta_{a b} z \delta(y+z) .
$$

For simplicity we assume that $\psi_{0}^{0}=v_{0}$ (if $n$ is odd). The elements $\psi_{0}^{ \pm a}(1 \leq a \leq m)$ are combinations of both creation and annihilation operators. To be more precise, there exist elements $u_{-1}, u_{-2}, \ldots, u_{-m} \in U_{0}$ and $u_{1}, u_{2}, \ldots, u_{m} \in\left(U_{0}+\mathbb{C} v_{0}\right)^{\perp}$ such that

$$
\begin{aligned}
& \left(u_{j}, \psi_{i}^{a}\right)=0 \quad \text { for all } a \text { and } i \neq 0, \\
& \left(u_{i}, u_{j}\right)=\delta_{i,-j}
\end{aligned}
$$

and

$$
\psi_{0}^{j}= \begin{cases}\frac{1}{2} \sqrt{2}\left(u_{j}+u_{-j}\right) & j>0, \\ \frac{i}{2} \sqrt{2}\left(u_{-j}-u_{j}\right) & j<0 .\end{cases}
$$

Then

$$
\psi_{0}^{j}|0\rangle=\left\{\begin{array}{cc}
\frac{1}{\sqrt{2}} u_{j}|0\rangle & j>0, \\
\frac{i}{\sqrt{2}} u_{j}|0\rangle & j<0, \\
-\frac{1}{\sqrt{2}}|0\rangle & j=0 .
\end{array}\right.
$$

As in the previous section we introduce

$$
\alpha^{a}(z)=\sum_{k \in 2 \mathbb{Z}+1} \alpha_{k}^{a} z^{-k-1}=: \psi(z) \frac{\psi(-z)}{z}: .
$$

Using (3.14) we find

$$
\begin{aligned}
{\left[\psi^{a}(y), \alpha^{b}(z)\right] } & =\delta_{a b}(\psi(-z) \delta(y+z)-\psi(z) \delta(y-z)), \\
{\left[\alpha^{a}(y), \alpha^{b}(z)\right] } & =\delta_{a b}\left(\delta_{z}^{\prime}(y-z)-\delta_{z}^{\prime}(y+z)\right) .
\end{aligned}
$$

The fermionic fields can be expressed in terms of the oscillators $\alpha_{k}^{a}$. However, in the multicomponent case one needs some extra operators $Q_{a}, 0 \leq|a| \leq m$, which are uniquely defined by

$$
\begin{aligned}
Q_{a} \psi_{n}^{b} & =-(-)^{\delta_{a b}} \psi_{n}^{b} Q_{a} \\
Q_{a}|0\rangle & =\sqrt{2} \psi_{0}^{a}|0\rangle .
\end{aligned}
$$

It is straightforward to check that

$$
\begin{aligned}
& Q_{a} Q_{b}+Q_{b} Q_{a}=2 \delta_{a b} \\
& Q_{a} \alpha_{m}^{b}-\alpha_{m}^{b} Q_{a}=0 .
\end{aligned}
$$

Notice that the space of highest weight vectors for the oscillator algebras, i.e., $f \in F$ such that $\alpha_{k}^{a} f=0$ for all $a$ and $k>0$ is $2^{m}$-dimensional. It has as basis elements the set

$$
Q_{1}^{k_{1}} Q_{2}^{k_{2}} \ldots Q_{m}^{k_{m}}|0\rangle=u_{1}^{k_{1}} u_{2}^{k_{2}} \ldots u_{m}^{k_{m}}|0\rangle, \quad \text { where } k_{i}=0 \text { or } 1 .
$$

One now has 
Theorem $3.3([18])$

$$
\psi^{a}(z)=\frac{Q_{a}}{\sqrt{2}} \exp \left(-\sum_{k<0, o d d} \frac{\alpha_{k}^{a}}{k} z^{-k}\right) \exp \left(-\sum_{k>0, o d d} \frac{\alpha_{k}^{a}}{k} z^{-k}\right) .
$$

We will now describe the $n$-component neutral (or twisted) boson-fermion correspondence. It caries the name twisted, because the expressions in Theorem 3.3 will be related to the twisted vertex operators as described in [5], [9].

Let, as in section $2.2, \mathfrak{L}$ be the lattice over $\mathbb{Z}$ with basis $\delta_{a}, \quad 1 \leq a \leq m$ and symmetric bilinear form $\left(\delta_{a} \mid \delta_{b}\right)=\delta_{a b}$. Let $\delta=\delta_{1}+\delta_{2}+\ldots+\delta_{m}$ and let $\epsilon: \mathfrak{L} \times \mathfrak{L} \rightarrow\{ \pm 1\}$ be the bimultiplicative function defined by (3.5), (3.6). Let $\mathfrak{K}$ be the even sublattice with basis $2 \delta_{a}, \quad 1 \leq a \leq m$. Then $\varepsilon$ induces a bimultiplicative function, which we also denote by $\varepsilon$, on $\mathfrak{L} / \mathfrak{K}$. Consider the vector space $\mathbb{C}_{\epsilon}[\mathfrak{L} / \mathfrak{K}]$ with basis $e^{\gamma}, \gamma \in \mathfrak{L} / \mathfrak{K}$ and the following twisted group algebra product

$$
e^{\alpha} e^{\beta}=\epsilon(\alpha, \beta) e^{\alpha+\beta} .
$$

Let $B=\mathbb{C}[t] \otimes_{\mathbb{C}} \mathbb{C}_{\epsilon}[\mathfrak{L} / \mathfrak{K}]$ where $\mathbb{C}[t]$ is the space of polynomials in the indeterminates $t_{k}^{(a)}$, $k=1,3,5, \ldots, 1 \leq a \leq n$. Then the neutral or twisted boson-fermion correspondence is the vector space isomorphism $\sigma: F \rightarrow B$

given by

$$
\begin{aligned}
& \sigma\left(\alpha_{-m_{1}}^{a_{1}} \alpha_{-m_{2}}^{a_{2}} \ldots \alpha_{-m_{s}}^{a_{s}} Q_{1}^{k_{1}} Q_{2}^{k_{2}} \ldots Q_{m}^{k_{m}}|0\rangle\right)= \\
& m_{1} m_{2} \ldots m_{s} t_{m_{1}}^{\left(a_{1}\right)} t_{m_{2}}^{\left(a_{2}\right)} \ldots t_{m_{s}}^{\left(a_{s}\right)} \otimes e^{k_{1} \delta_{1}+\kappa_{2} \delta_{2}+\ldots+k_{m} \delta_{m}} .
\end{aligned}
$$

One has the following isomorphism

$$
\sigma\left(F_{\bar{\nu}}\right)=B_{\bar{\nu}}
$$

where $B_{\bar{\nu}}$ are the eigenspaces of the operator $1 \otimes(-1)^{\delta}$, i.e. $B=B_{\overline{0}}+B_{\overline{1}}$ with

$$
B_{\bar{\nu}}=\left\{b \in B \mid 1 \otimes(-1)^{\delta} b=(-)^{\nu} b\right\} .
$$

The transported action of the operators is as follows $(k>0,1 \leq a<m, 0 \leq|b| \leq m)$ :

$$
\begin{aligned}
\sigma \alpha_{-k}^{b} \sigma^{-1}\left(p(t) \otimes e^{\gamma}\right) & =k t_{k}^{(b)} p(t) \otimes e^{\gamma}, \\
\sigma \alpha_{k}^{b} \sigma^{-1}\left(p(t) \otimes e^{\gamma}\right) & =2 \frac{\partial p(t)}{\partial t_{k}^{(b)}} \otimes e^{\gamma}, \\
\sigma Q_{a} \sigma^{-1}\left(p(t) \otimes e^{\gamma}\right) & =\epsilon\left(\delta_{a}, \gamma\right) p(t) \otimes e^{\gamma+\delta_{a}}, \\
\sigma Q_{-a} \sigma^{-1}\left(p(t) \otimes e^{\gamma}\right) & =i(-)^{\left(\delta_{a} \mid \gamma\right)} \epsilon\left(\delta_{a}, \gamma\right) p(t) \otimes e^{\gamma+\delta_{a}}, \\
\sigma Q_{0} \sigma^{-1}\left(p(t) \otimes e^{\gamma}\right) & =-(-)^{(\delta \mid \gamma)} p(t) \otimes e^{\gamma} .
\end{aligned}
$$

From now on we omit the tensor symbol $\otimes$ and write as before $\sigma Q_{a} \sigma^{-1}=e^{\delta_{a}}$ for $0 \leq a \leq$ $m$. Then one has for $0 \leq a \leq m$ :

$$
\begin{aligned}
\sigma \psi^{a}(z) \sigma^{-1} & =\frac{e^{\delta_{a}}}{\sqrt{2}} \exp \left(\xi_{a}(t, z) \exp \left(-\eta_{a}(t, z)\right),\right. \\
\sigma \psi^{0}(z) \sigma^{-1} & =\frac{-(-)^{\delta}}{\sqrt{2}} \exp \left(\xi_{0}(t, z) \exp \left(-\eta_{0}(t, z)\right) \quad \text { if } n\right. \text { is odd, } \\
\sigma \psi^{-a}(z) \sigma^{-1} & =\frac{i e^{\delta_{a}}}{\sqrt{2}}(-)^{\delta_{a}} \exp \left(\xi_{-a}(t, z) \exp \left(-\eta_{-a}(t, z)\right),\right.
\end{aligned}
$$


where

$$
\xi_{b}(t, z)=\sum_{k=1}^{\infty} t_{2 k-1}^{(b)} z^{2 k-1} \quad \text { and } \quad \eta_{b}(t, z)=\sum_{k=1}^{\infty} \frac{2}{2 k-1} \frac{\partial}{\partial t_{2 k-1}^{(b)}} z^{1-2 k}
$$

\subsection{General case}

The general case will be a combination of $n$ charged fermions and $n^{\prime}$ neutral fermions, hence it combines the sections 3.1 and 3.3. As before we assume that $n^{\prime}=2 m+1$ if $n^{\prime}$ is odd and $n^{\prime}=2 m$ if $n^{\prime}$ is even. For notational convenience, we introduce three sets $C F, N F$ and $S F$ (Charged, Neutral and Simple Fermions):

$$
\begin{aligned}
& N F= \begin{cases}\{0, \pm 1, \pm 2, \ldots, \pm m\} & \text { if } n^{\prime} \text { is odd, } \\
\{ \pm 1, \pm 2, \ldots, \pm m\} & \text { if } n^{\prime} \text { is even, }\end{cases} \\
& C F=\{ \pm(m+1), \pm(m+2), \ldots, \pm(m+n)\}, \\
& S F= \begin{cases}\{0\} & \text { if } n^{\prime} \text { is even, } \\
\emptyset & \text { otherwise. }\end{cases}
\end{aligned}
$$

We choose the basis elements $\psi_{i}^{a}$ of $V$, where $i \in \frac{1}{2}+\mathbb{Z}, i \in \mathbb{Z}, i=0$ for $a \in C F, N F, S F$, respectively. These $\psi_{i}^{a}$ satisfy

$$
\left(\psi_{i}^{a}, \psi_{j}^{b}\right)= \begin{cases}(-)^{i} \delta_{a, b} \delta_{i,-j} & \text { if } a \in N F \\ \delta_{a,-b} \delta_{i,-j} & \text { if } a \in C F \\ \delta_{a b} \delta_{0, j} & \text { if } a \in S F \text { with } i=0 .\end{cases}
$$

Moreover we assume that

$$
\psi_{i}^{a} \in U_{0} \text { if } i>0 \quad \psi_{0}^{0}=v_{0},
$$

and that the $\psi_{0}^{a}, a \neq 0$, are given by (3.19), where $u_{-1}, u_{-2}, \ldots, u_{-m} \in U_{0}$ and $u_{1}, u_{2}, \ldots, u_{m} \in$ $\left(U_{0} \oplus \mathbb{C} v_{0}\right)^{\perp}$ are linearly independent andsatisfy (3.18). Introduce the fermionic fields $\psi^{a}(z)$ by

$$
\psi^{a}(z)= \begin{cases}\sum_{i \in \frac{1}{2}+\mathbb{Z}} \psi_{i}^{a} z^{-i-\frac{1}{2}} & \text { for } a \in C F \\ \sum_{i \in \mathbb{Z}} \psi_{i}^{a} z^{-i} & \text { for } a \in N F .\end{cases}
$$

The commutators $\left[\psi^{a}(y), \psi^{b}(z)\right]$ (resp. $\left.\psi^{a}(y) \frac{\psi^{b}(-z)}{z}\right)$ for $a \in C F$ (resp. $a \in N F$ ) are given by (3.3) (resp. (3.17)). Introduce as before the oscillator algebra

$$
\alpha^{a}(z)= \begin{cases}\sum_{i \in \mathbb{Z}} \alpha_{i}^{a} z^{-i-1}=: \psi^{a}(z) \psi^{-a}(z): & \text { for } a \in C F, \\ \sum_{i \in 2 \mathbb{Z}+1} \alpha_{i}^{a} z^{-i-1}=: \psi^{a}(z) \frac{\psi^{a}(-1)}{z}: & \text { for } a \in N F .\end{cases}
$$

Then the commutators $\left[\psi^{a}(y), \alpha^{b}(z)\right]$ and $\left[\alpha^{a}(y,) \alpha^{b}(z)\right]$ for $a \in C F$ (resp. $a \in N F$ ) are given by (3.4) (resp. (3.22)). 
In order to express the fermionic fields in terms of the oscillator algebra, we have to introduce,as before, some new operators $Q_{a},-m \leq a \leq m+n, a \neq 0$ and we let, for notational convenience, that $Q_{0}=\sqrt{2} v_{0}$ if $0 \in S F$. These $Q_{a}$ are uniquely defined by

$$
\begin{aligned}
Q_{a} \psi_{i}^{b} & =\begin{aligned}
-\psi_{i}^{b} Q_{a} & \text { for } a \neq|b|,
\end{aligned} \\
Q_{a} \psi_{i}^{ \pm a} & =\left\{\begin{aligned}
\psi_{i \mp s(a)}^{ \pm a} Q_{a} & \text { for } a \in C F, \\
\pm \psi_{i}^{ \pm a} Q_{a} & \text { for } a \in N F, \\
\psi_{0}^{0} Q_{0}, &
\end{aligned}\right. \\
Q_{0} \psi_{0}^{0} & =\left\{\begin{aligned}
\psi_{-\frac{1}{2}}^{a}|0\rangle & \text { for } a \in C F, \\
\psi_{0}^{a}|0\rangle & \text { for } a \notin C F .
\end{aligned}\right. \\
Q_{a}|0\rangle & =\{
\end{aligned}
$$

So they satisfy the following commutation relations

$$
\begin{array}{ll}
Q_{a} Q_{b}+Q_{b} Q_{a}=0 & \text { for } a \neq b, \\
Q_{a}^{2}=1 & \text { if } a \notin C F . \\
{\left[\alpha_{k}^{a}, Q_{b}\right]=\delta_{k, 0} \delta_{a b} Q_{b}} &
\end{array}
$$

Theorem 3.4 Let $a \in C F a>0, b \in N F$, then

$$
\begin{aligned}
\psi^{ \pm a}(z) & =Q_{a}^{ \pm 1} z^{ \pm \alpha_{0}^{a}} \exp \left(\mp \sum_{k<0} \frac{\alpha_{k}}{k} z^{-k}\right) \exp \left(\mp \sum_{k>0} \frac{\alpha_{k}}{k} z^{-k}\right), \\
\psi^{b}(z) & =\frac{Q_{b}}{\sqrt{2}} \exp \left(-\sum_{k<0, o d d} \frac{\alpha_{k}^{b}}{k} z^{-k}\right) \exp \left(-\sum_{k>0, o d d} \frac{\alpha_{k}^{b}}{k} z^{-k}\right) .
\end{aligned}
$$

The space of highest weight vectors for the oscillator algebra, i.e. $f \in F$ such that $\alpha_{k}^{a} f=0$ for all $k>0$, has as basis the following vectors

$$
Q_{1}^{k_{1}} Q_{2}^{k_{2}} \ldots Q_{m+n}^{k_{m+n}} \mid 0>
$$

where $k_{i}= \pm 1$ for $i=1,2, \ldots, m$ and $k_{i} \in \mathbb{Z}$ for $i=m+1, m+2, \ldots, m+n$.

In a similar way as in the previous sections we introduce the lattice $\mathfrak{L}$ over $\mathbb{Z}$ with basis $\delta_{a}$, $a=1,2, \ldots, m+n$ and symmetric bilinear form $\left(\delta_{a} \mid \delta_{b}\right)=\delta_{a b}$. Let $\delta=\delta_{1}+\delta_{2}+\ldots+\delta_{n+m}$ and let $\epsilon: \mathfrak{L} \times \mathfrak{L} \rightarrow\{ \pm 1\}$ be the bimultiplicative function defined by (3.6 - 3.7). Let $\mathfrak{K}$ be the even sublattice of $\mathfrak{L}$ generated by $2 \delta_{a} \quad 1 \leq a \leq m$. Then $\epsilon$ induces a bimultiplicative function, which we also denote by $\epsilon$, on $\mathfrak{L} / \mathfrak{K}$.

It will be convenient to introduce the sublattice $\mathfrak{N}=\mathfrak{N}_{\overline{0}}=\{\alpha \in \mathfrak{L} \mid(\alpha \mid \delta) \in 2 \mathbb{Z}\}$ and let $\mathfrak{N}_{\overline{1}}=\{\alpha \in \mathfrak{L} \mid(\alpha \mid \delta) \in 2 \mathbb{Z}+1\}$. Then $\mathfrak{N} / \mathfrak{K}$ is well defined. From now on we will use the notation $\overline{\mathfrak{L}}, \overline{\mathfrak{L}}_{\overline{0}}$ and $\overline{\mathfrak{L}}_{\overline{1}}$ for $\mathfrak{L} / \mathfrak{K},, \mathfrak{N} / \mathfrak{K}, \mathfrak{N} \overline{\overline{1}} / \mathfrak{K}$ respectively. If $n^{\prime}=0$ we also introduce

$$
\mathfrak{M}_{k}=\{\alpha \in \mathfrak{L} \mid(\alpha \mid \delta)=k\},
$$

then notice that $\mathfrak{M}_{0}$ is the root lattice of $s \ell_{n}$. The sets $\overline{\mathfrak{L}}, \overline{\mathfrak{L}}_{\overline{0}}$ and $\overline{\mathfrak{L}}_{\overline{1}}$ and $\mathfrak{M}_{k}$ will be related to $F, F_{\overline{0}}, F_{\overline{1}}, F_{k}$, respectively.

Let $B=\mathbb{C}[t] \otimes_{\mathbb{C}} \mathbb{C}_{\epsilon}[\overline{\mathfrak{L}}]$ where $\mathbb{C}[t]$ is the space of polynomials in the indeterminates $t=$ $\left\{t_{k}^{(a)}\right\}, k=1,2, \ldots$ for $0<a \in C F$ and $k=1,3,5$ for $a \in N F$. The orthogonal bosonfermion correspondence is the isomorphism $\sigma: F \stackrel{\sim}{\rightarrow} B$ given by

$$
\begin{aligned}
& \sigma\left(\alpha_{-m_{1}}^{a_{1}} \alpha_{-m_{2}}^{a_{2}} \ldots \alpha_{-m_{s}}^{a_{s}} Q_{1}^{k_{1}} Q_{2}^{k_{1}} \ldots Q_{n+m}^{k_{n+m}}|0\rangle\right)= \\
& m_{1} m_{2} \ldots m_{s} t_{m_{1}}^{\left(a_{1}\right)} t_{m_{2}}^{\left(a_{2}\right)} \ldots t_{m_{s}}^{\left(a_{s}\right)} e^{k_{1} \delta_{1}+k_{2} \delta_{2}+\ldots+k_{n+m} \delta_{n+m}} .
\end{aligned}
$$


The transported operators are then given by (3.25) for $|a|<m$ and (3.9) for $a=m+$ $1, \ldots, m+n$. Then the formulas for the transported fields $\sigma \psi^{a}(z) \sigma^{-1}$ are given by $(3.26)$ and (3.27) for $|a|<m$ and (3.11) and (3.12) for $a \in C F$.

\section{Multicomponent BKP and DKP hierarchies; bilinear equa- tions for the $\tau$-functions and the wave functions}

Using the orthogonal boson-fermion correspondence, we can reformulate the BKP (1.18), DKP (1.19), Modified DKP (1.20), KP and modified KP hierarchies (2.16) as systems of Hirota bilinear equations. The BKP hierarchy contains all the equations of all the other hierarchies. The DKP, KP and first modified KP hierarchies can be obtained by a reduction of BKP when $n^{\prime}$ is even, $n^{\prime}=0$ and $n^{\prime}=0$, respectively. The boson-fermion correspondece in the $n^{\prime}$ odd case does not allow to express the operators $S^{\prime}$ in terms of fermionic fields. Reduction to the KP and modified KP hierarchies are only possible for $n^{\prime}=0$, since only then it is possible to express $S^{+}$and $S^{-}$in terms of the fermionic fields.

It will be convenient to make a distinction between $n^{\prime}$ even and $n^{\prime}$ odd. For that purpose we introduce

$$
\varepsilon\left(n^{\prime}\right)=\left\{\begin{array}{llll}
0 & \text { if } & n^{\prime} & \text { is even, } \\
1 & \text { if } & n^{\prime} & \text { is odd. }
\end{array}\right.
$$

Observe that we can rewrite the BKP hierarchy $(1.18)$ in terms of the fermionic fields as follows:

$$
\begin{gathered}
\operatorname{Res}_{z=0} d z\left\{\left(1-\epsilon\left(n^{\prime}\right)\right) \frac{\psi_{0}^{0} \otimes \psi_{0}^{0}}{z}+\epsilon\left(n^{\prime}\right) \psi^{0}(z) \otimes \frac{\psi^{0}(-z)}{z}\right. \\
+\sum_{a=1}^{m}\left(\psi^{a}(z) \otimes \frac{\psi^{a}(z)}{-z}+\psi^{-a}(z) \otimes \frac{\psi^{-a}(-z)}{z}\right) \\
\left.+\sum_{a=m+1}^{m+n}\left(\psi^{a}(z) \otimes \psi^{-a}(z)-\psi^{-a}(-z) \otimes \psi^{a}(-z)\right)\right\} \tau \otimes \tau \\
=\frac{1}{2} \tau \otimes \tau .
\end{gathered}
$$

We write $\sigma(\tau)=\sum_{\gamma \in \mathfrak{L}} \tau_{\gamma}(t) e^{\gamma}$, then using the orthogonal boson-fermion correspondence of section 3.4, we rewrite (4.1) and take the coefficient of $e^{\alpha} \otimes e^{\beta}$, for every $\alpha, \beta \in \overline{\mathfrak{L}}$. One thus obtains the following equation:

$$
\begin{aligned}
& \operatorname{Res}_{z=0} d z\left\{\frac{1-\epsilon\left(n^{\prime}\right)}{2 z}\left((-)^{(\delta \mid \alpha+\beta)}-1\right) \tau_{\alpha} \otimes \tau_{\beta}\right. \\
+ & \frac{\epsilon\left(n^{\prime}\right)}{2 z}\left((-)^{(\delta \mid \alpha)} E^{0}(t, z) \tau_{\alpha} \otimes(-)^{(\delta \mid \beta)} E^{0}(t,-z) \tau_{\beta}-\tau_{\alpha} \otimes \tau_{\beta}\right) \\
+ & \frac{1}{2 z} \sum_{a=1}^{m} \epsilon\left(\delta_{a}, \alpha\right) E^{a}(t, z) \tau_{\alpha-\delta_{a}} \otimes \epsilon\left(\delta_{a}, \beta\right) E^{a}(t,-z) \tau_{\beta+\delta_{a}} \\
- & \frac{1}{2 z} \sum_{a=1}^{m} \epsilon\left(\delta_{a}, \alpha\right)(-)^{\left(\delta_{a} \mid \alpha\right)} E^{-a}(t, z) \tau_{\alpha+\delta_{a}} \otimes \epsilon\left(\delta_{a}, \beta\right)(-)^{\left(\delta_{a} \mid \beta\right)} E^{-a}(t,-z) \tau_{\beta-\delta_{a}} \\
+ & \sum_{a=m+1}^{m+n} \epsilon\left(\delta_{a}, \alpha\right) z^{\left(\delta_{a} \mid \alpha\right)-1} E_{+}^{a}(t, z) \tau_{\alpha-\delta_{a}} \otimes \epsilon\left(\delta_{a}, \beta\right) z^{-\left(\delta_{a} \mid \beta\right)-1} E_{-}^{a}(t,-z) \tau_{\beta+\delta_{a}} \\
- & \left.\sum_{a=m+1}^{m+n} \epsilon\left(\delta_{a}, \alpha\right)(-z)^{-\left(\delta_{a} \mid \alpha\right)-1} E_{-}^{a}(t, z) \tau_{\alpha+\delta_{a}} \otimes \epsilon\left(\delta_{a}, \beta\right)(-z)^{\left(\delta_{a} \mid \beta\right)-1} E_{+}^{a}(t,-z) \tau_{\beta-\delta_{a}}\right\} \\
\quad & =0 .
\end{aligned}
$$


Here

$$
\begin{aligned}
E^{a}(t, z) & =e^{\xi_{a}(t, z)} e^{-\eta_{a}(t, z)} \\
& =e^{\sum_{k=1}^{\infty} t_{2 k-1}^{(a)} z^{2 k-1}} e^{-\sum_{k=1}^{\infty} \frac{\partial}{\partial t_{2 k-1}^{(a)}} \frac{z^{1-2 k}}{2 k-1}} \text { for }|a| \leq m \\
E_{ \pm}^{a}(t, z) & =e^{ \pm \xi_{a}(t, \pm z)} e^{\mp \eta_{a}(t, \pm z)} \\
& =e^{ \pm \sum_{k=1}^{\infty} t_{k}^{(a)}( \pm z)^{k}} e^{\mp \sum_{k=1}^{\infty} \frac{\partial}{\partial t_{k}^{(a)}} \frac{( \pm z)-k}{k}} \text { for } a>m .
\end{aligned}
$$

Further notice that $\tau_{\alpha-\delta_{a}}=\tau_{\alpha+\delta_{a}}$ for $|a| \leq m$. Identifying $\mathbb{C}[t] \otimes \mathbb{C}[t]$ with $\mathbb{C}\left[t^{\prime}, t^{\prime \prime}\right]$, where we write $t^{\prime}$ (resp. $t^{\prime \prime}$ ) for the first (resp. second) component of the tensor product. Making the change of variables

$$
t_{k}^{(a)}=\frac{1}{2}\left(t_{k}^{(a)^{\prime}}+t_{k}^{(a)^{\prime \prime}}\right) \quad s_{k}^{(a)}=\frac{1}{2}\left(t_{k}^{(a)^{\prime}}-t_{k}^{(a)^{\prime \prime}}\right)
$$

(4.2) becomes:

$$
\begin{aligned}
& \operatorname{Res}_{z=0} d z\left\{\left(\frac{(-)^{\left(\delta_{a} \mid \alpha+\beta\right)}-1}{2 z}+\frac{\epsilon\left(n^{\prime}\right)}{2 z}(-)^{\left(\delta_{a} \mid \alpha+\beta\right)}\left(e^{\xi_{0}(2 s, z)} e^{-\eta_{0}(s, z)}-1\right)\right) \times\right. \\
& \tau_{\alpha}(t+s) \tau_{\beta}(t-s)+ \\
+ & \frac{1}{2 z} \sum_{a=1}^{m} \epsilon\left(\delta_{a}, \alpha+\beta\right)\left(e^{\xi_{a}(2 s, z)} e^{-\eta_{a}(s, z)}-(-)^{\left(\delta_{a} \mid \alpha+\beta\right)} e^{\xi_{-a}(2 s, z)} e^{-\eta_{-a}(s, z)}\right) \times \\
& \tau_{\alpha-\delta_{a}}(t+s) \tau_{\alpha+\delta_{b}}(t-s)+ \\
+ & \sum_{a=m+1}^{m+n} \epsilon\left(\delta_{a}, \alpha+\beta\right) z^{\left(\delta_{a} \mid \alpha-\beta\right)-2} e^{\xi_{a}(2 s, z)} e^{-\eta_{a}(s, z)} \tau_{\alpha-\delta_{a}}(s+t) \tau_{\beta+\delta_{a}}(t-s)+ \\
+ & \left.\sum_{a=m+1}^{m+n} \epsilon\left(\delta_{a}, \alpha+\beta\right) z^{\left(\delta_{a} \mid \beta-\alpha\right)-2} e^{-\xi(2 s, z)} e^{\eta_{a}(s, z)} \tau_{\alpha+\delta_{a}}(s+t) \tau_{\beta-\delta_{a}}(t-s)\right\}=0 .
\end{aligned}
$$

Here we have used the fact that $\operatorname{Res}_{z=0} d z f(-z)=-\operatorname{Res}_{z=0} d z f(z)$. We can rewrite (4.6) using elementary Schur polynomials, which are defined by (1.13):

$$
\begin{aligned}
& \left(\frac{(-)^{\left(\delta_{a} \mid \alpha+\beta\right)-1}}{2}+\frac{\epsilon\left(n^{\prime}\right)}{2}(-)^{\left(\delta_{a} \mid \alpha+\beta\right)}\left(\sum_{k=0}^{\infty} P_{k}\left(2 s^{(0)}\right) P_{k}\left(2 s^{(0)}\right) P_{k}\left(-2 \frac{\tilde{\partial}}{\partial s^{(0)}}\right)-1\right)\right) \times \\
& \tau_{\alpha}(t+s) \tau_{\beta}(t-s)+ \\
+ & \frac{1}{2} \sum_{a=1}^{m} \epsilon\left(\delta_{a}, \alpha+\beta\right) \sum_{k=0}^{\infty} P_{k}\left(2 s^{(a)}\right) P_{k}\left(-2 \frac{\tilde{\partial}}{\partial s^{(a)}}\right)-(-)^{\left(\delta_{a} \mid \alpha+\beta\right)} P_{k}\left(2 s^{(-a)}\right) \times \\
& P_{k}\left(-2 \frac{\tilde{\partial}}{\partial S^{(-a)}}\right) \tau_{\alpha-\delta_{a}}(s+t) \tau_{\beta+\delta}(t-s)+ \\
+\quad & \sum_{a=m+1}^{m+n} \epsilon\left(\delta_{a}, \alpha+\beta\right) \sum_{k=0}^{\infty} P_{k}\left(2 s^{(a)}\right) P_{k-1+\left(\delta_{a} \mid \alpha-\beta\right)}\left(-\frac{\tilde{\partial}}{\partial s^{(a)}}\right) \times \\
& \tau_{\alpha-\delta_{a}}(s+t) \tau_{\beta+\delta_{a}}(t-s)+ \\
+\quad & \sum_{a=m+1}^{m+n} \epsilon\left(\delta_{a}, \alpha+\beta\right) \sum_{k=0}^{\infty} P_{k}\left(-2 s^{(a)}\right) P_{k-1+\left(\delta_{a} \mid \beta-\alpha\right)}\left(\frac{\tilde{\partial}}{\partial s^{(a)}}\right) \times \\
& \tau_{\alpha+\delta_{a}}(s+t) \tau_{\beta-\delta_{a}}(t-s)=0 .
\end{aligned}
$$


where

$$
\frac{\tilde{\partial}}{\partial s^{(a)}}= \begin{cases}\left(\frac{\partial}{\partial S_{1}^{(a)}}, \frac{1}{2} \frac{\partial}{\partial S_{2}^{(a)}}, \frac{1}{3} \frac{\partial}{\partial S_{3}^{(a)}}, \ldots\right) & a>m, \\ \left(\frac{\partial}{\partial S_{1}^{(a)}}, \frac{1}{3} \frac{\partial}{\partial S_{3}^{(a)}}, \frac{1}{5} \frac{\partial}{\partial S_{5}^{(a)}}, \ldots\right) & |a| \leq m .\end{cases}
$$

Using Taylors formula we rewrite this once more:

$$
\begin{aligned}
& \left(\frac{(-)^{\left(\delta_{a} \mid \alpha+\beta\right)}-1}{2}+\frac{\epsilon\left(n^{\prime}\right)}{2}(-)^{\left(\delta_{a} \mid \alpha+\beta\right)}\left(\sum_{k=0}^{\infty} P_{k}\left(2 s^{(0)}\right) P_{k}\left(-2 \frac{\tilde{\partial}}{\partial u^{(0)}}\right)-1\right)\right) \times \\
& \left.D(s, u) \tau_{\alpha}(t+u) \tau_{\beta}(t-u)\right|_{u=0} \\
+ & \frac{1}{2} \sum_{a=1}^{m} \epsilon\left(\delta_{a}, \alpha+\beta\right) \sum_{k=0}^{\infty} P_{k}\left(2 s^{(a)}\right) P_{k}\left(-2 \frac{\tilde{\partial}}{\partial u^{(a)}}\right)-(-)^{\left(\delta_{a} \mid \alpha+\beta\right)} P_{k}\left(2 s^{(-a)}\right) \times \\
& \left.P_{k}\left(-2 \frac{\tilde{\partial}}{\partial u^{(-a)}}\right) D(s, u) \tau_{\alpha-\delta_{a}}(t+u) \tau_{\beta+\delta_{u}}(t-u)\right|_{u=0}+ \\
+\quad & \sum_{a=m+1}^{m+n} \epsilon\left(\delta_{a}, \alpha+\beta\right) \sum_{k=0}^{\infty} P_{k}\left(2 s^{(a)}\right) P_{k-1+\left(\delta_{a} \mid \alpha-\beta\right)}\left(-\frac{\tilde{\partial}}{\partial u^{(a)}}\right) D(s, u) \\
& \left.\tau_{\alpha-\delta_{a}}(t+u) \tau_{b+\delta_{a}}(t-u)\right|_{u=0}+ \\
+\quad & \sum_{a=m+n}^{m+n} \epsilon\left(\delta_{a}, \alpha+\beta\right) \sum_{k=0}^{\infty} P_{k}\left(-2 s^{(a)}\right) P_{k-1+\left(\delta_{a} \mid \beta-\alpha\right)}\left(\frac{\tilde{\partial}}{\partial u^{(a)}}\right) D(s, u) \times \\
& \left.\tau_{\alpha+\delta_{a}}(t+u) \tau_{\beta-\delta_{a}}(t-u)\right|_{u=0}=0 .
\end{aligned}
$$

where

$$
D(s, u)=e^{\sum_{a=1}^{m} \sum_{r=1}^{\infty} s_{2 r-1}^{(a)} \frac{\partial}{\partial u_{2 r-1}^{(a)}} \epsilon\left(n^{\prime}\right) \sum_{r=0}^{\infty} S_{2 r-1}^{(0)} \frac{\partial}{\partial u_{2 r-1}^{(0)}} e^{a=1} \sum_{r=1}^{m} s_{2 r-1}^{(-a)} \frac{\partial}{\partial u_{2 r-1}^{(-a)}} \sum^{a=m+1} \sum_{r}^{m+n} s_{r}^{(a)} \frac{\partial}{\partial u_{r}^{(a)}}}
$$

In fact (4.7) is a generating series of Hirota bilinear equations (see the Introduction).

The equations for the DKP and modified DKP hierarchies are only given if $n^{\prime}$ is even, so $\epsilon\left(n^{\prime}\right)=0$. Then the two DKP hierarchies are the set of equations for wich either $\alpha, \beta \in \overline{\mathfrak{L}}_{0}$ or $\alpha, \beta \in \overline{\mathfrak{L}}_{1}$. The modified DKP is the set of equations for which $\alpha \in \overline{\mathfrak{L}}_{\overline{0}}$ and $\beta \in \overline{\mathfrak{L}}_{\overline{1}}$ (or the other way around which gives the same set of equations). The equations for the KP or first modified KP hierarchy appear only if $n^{\prime}=m=\epsilon\left(n^{\prime}\right)=0$ and one assumes that $\tau_{\alpha}=0$ for all $\alpha$ except $\alpha \in \mathfrak{M}_{k}$, respectively $\alpha \in \mathfrak{M}_{k} \cup \mathfrak{M}_{k+1}$. It is straightforward to see that for those $\tau$ 's the equations (4.7) decouple into two sets of equations, which are in fact equivalent by Theorem (2.3).

We wil now give the bilinear equation for the wave functions. Following T. Shiota [16] in which he describes wave functions for the 2 component BKP hierarchy, we are lead in this general case ( $n, n^{\prime}$ non-negative integers) to introduce two wave functions. But before doing that it will be convenient to replace in (4.9) $t_{j}^{(a)}$ by $t_{j}^{(a)}+\delta_{j 1} x_{0}$ if $a \geq 0$ and by $t_{j}^{(a)}+\delta_{j 1} x_{1}$ if $a<0$, then of course $\tau_{\gamma}$ will be a function of all $t_{j}^{(a)}$ 's and $x_{0}, x_{1}$, we will use the notation $\tau_{\gamma}(x, t)$.

We will reformulate (4.2) in such a way that we obtain expressions involving matrix valued formal pseudodifferential operators in $\partial_{0}=\frac{\partial}{\partial x_{0}}$ and $\partial_{1}=\frac{\partial}{\partial x_{1}}$.

Introduce

$$
P^{ \pm \sigma}(\alpha, x, t, \pm z)=\left(P^{ \pm \sigma}(\alpha, x, t, \pm z)_{j k}\right)_{-n \leq j, k \leq n+m}
$$


for $\sigma=0,1$ as follows. Let $\lambda=+,-$ and assume $j=1,2, \ldots, m+n$ if $\lambda=+$ and $j=m+1, m+2, \ldots, m+n$ if $\lambda=-$, then define

$$
\begin{aligned}
& P^{ \pm 0}(\alpha, x, t, \pm z)_{\lambda j+\delta_{\lambda,-} m, k}= \\
& \left\{\begin{array}{cc}
\frac{\epsilon_{k j} e^{-\eta_{k}(t, \pm z)} \tau_{a \pm \lambda \delta_{j} \mp \delta_{k}}(x, t)}{\tau_{\alpha}(x, t)} & \text { if } \quad k=1, \ldots, m, \\
\frac{\epsilon_{k j} z^{\lambda \delta_{j k}-1} e^{\mp \eta_{k}(t, z)} \tau_{a \pm \lambda \delta_{j} \mp \delta_{k}}(x, t)}{\tau_{\alpha}(x, t)} & \text { if } \quad k=m+1, \ldots, m+n, \\
-\frac{\left(1-\epsilon\left(n^{\prime}\right)\right) \tau_{a \pm \lambda \delta_{j}}(x, t)-\epsilon\left(n^{\prime}\right) e^{-\eta_{0}(t, \pm z)} \tau_{\alpha \pm \lambda \delta_{j}}(x, t)}{\tau_{\alpha}(x, t)} & \text { if } \quad k=0, \\
\frac{\epsilon_{j, m-k} z^{-\lambda \delta_{j, m-k}-1} e^{ \pm \eta_{m-k}(t,-z)} \tau_{\alpha \pm \lambda \delta_{j} \pm \delta_{m-k}}(x, t)}{\tau_{\alpha}(x, t)} & \text { if } \quad k=-1, \ldots,-n, \\
P^{ \pm 0}(\alpha, x, t, \pm z)_{0, k}= & \text { if } \quad k=1, \ldots, m, \\
\frac{e^{-\eta_{k}(t, \pm z)} \tau_{\alpha \mp \delta_{k}}(x, t)}{\tau_{\alpha}(x, t)} & \text { if } \quad k=m+1, \ldots, m+n, \\
\frac{z^{-1} e^{\mp \eta_{k}(t, z)} \tau_{\alpha \mp \delta_{k}}(x, t)}{\tau_{a}(x, t)} & \text { if } \quad k=0, \\
1-\epsilon\left(n^{\prime}\right)+\epsilon\left(n^{\prime}\right) \frac{e^{-\eta_{0}(t, \pm z)} \tau_{\alpha}(x, t)}{\tau_{\alpha}(x, t)} & \text { if } \quad k=1, \ldots,-n, \\
-\frac{z^{-1} e^{ \pm \eta_{m-k}(t,-z)} \tau_{a \pm \delta_{m-k}}(x, t)}{\tau_{a}(x, t)} &
\end{array}\right.
\end{aligned}
$$

$$
P^{ \pm 1}(\alpha, x, t, \pm z)_{j k}=\left\{\begin{array}{cll}
\frac{\epsilon_{j k} e^{-\eta}-k(t, \pm z)}{\tau_{\alpha \pm \delta_{j} \mp \delta_{k}}(x, t)} & \text { if } \quad k=1, \ldots, m, \\
\delta_{j k}(x, t) & \text { if } \quad k=m+1, \ldots, m+n, \\
\frac{\tau_{\alpha \pm \delta_{j}}(x, t)}{\tau_{\alpha}(x, t)} & \text { or } \quad k=-1, \ldots,-n, \\
& \text { if } \quad k=0,
\end{array}\right.
$$

for $j=1,2, \ldots, m+n$ and

$$
\begin{gathered}
P^{ \pm 1}(\alpha, x, t, \pm z)_{0 k}=\left\{\begin{array}{cl}
\delta_{k 0} & \text { if } \quad k \neq 1,2, \ldots, m, \\
\frac{e^{-\eta_{-k}(t, \pm z)} \tau_{\alpha \mp \delta_{k}}(x, t)}{\tau_{\alpha}(x, t)} & \text { if } \quad k=1, \ldots, m,
\end{array}\right. \\
P^{ \pm 1}(\alpha, x, t, \pm z)_{j k}=\left\{\begin{array}{cc}
\delta_{j k} & \text { if } \quad k \neq 0, \\
\frac{\tau_{\alpha \mp} \delta_{m-j}(x, t)}{\tau_{\alpha}(x, t)} & \text { if } \quad k=0,
\end{array}\right.
\end{gathered}
$$

for $j=-1,-2, \ldots,-n$.

Next define $Q^{ \pm \sigma}(t, \pm z), R^{ \pm \sigma}(\alpha, \pm z) \quad(\sigma=0,1)$ and $S(z)$ as follows: 


$$
\begin{aligned}
Q^{ \pm 0}(t, \pm z)= & \sum_{j=1}^{m+n} e^{ \pm \xi_{j}(t, z)} E_{j j}+\left(1-\epsilon\left(n^{\prime}\right)+\epsilon\left(n^{\prime}\right) e^{ \pm \xi_{0}(t, z)}\right) E_{00}+ \\
& +\sum_{j=-n}^{-1} e^{\mp \xi_{m-j}(t,-z)} E_{j j}, \\
Q^{ \pm 1}(t, \pm z)= & \sum_{j=1}^{m} e^{ \pm \xi_{-j}(t, z)} E_{j j}+\sum_{j=m+1}^{m+n} E_{j j}+\sum_{j=-n}^{-1} E_{j j}, \\
R^{ \pm 0}(\alpha, \pm z)= & \sum_{j=1}^{m} \epsilon\left(\delta_{j}, \alpha\right) E_{j j}+\sum_{j=m+1}^{m+n} \epsilon\left(\delta_{j}, \alpha\right) z^{ \pm\left(\delta_{j} \mid \alpha\right)} E_{j j} \\
& +(-)^{(\delta \mid \alpha)} E_{00}+\sum_{j=-n}^{-1} \epsilon\left(\delta_{m-j}, \alpha\right)(-z)^{\mp\left(\delta_{m-j} \mid \alpha\right)} E_{j j}, \\
R^{ \pm 1}(\alpha, \pm z)= & \sum_{j=1}^{m} \epsilon\left(\delta_{j}, \alpha\right)(-)^{\left(\delta_{j} \mid \alpha\right)} E_{j j}+\sum_{j=m+1}^{m+n} E_{j j}+\sum_{j=-n}^{0} E_{j j}, \\
S(z)= & \frac{1}{2 z} \sum_{j=0}^{m} E_{j j}+\sum_{j=m+1}^{m+n} E_{j j}-\sum_{j=-n}^{-1} E_{j j} .
\end{aligned}
$$

And introduce the wave operators

$$
W^{ \pm \sigma}(\alpha, x, t, \pm z)=P^{ \pm \sigma}(\alpha, x, t, \pm z) Q^{ \pm \sigma}(t, \pm z) R^{ \pm \sigma}(\alpha, \pm z)
$$

and the wave functions

$$
\Psi^{ \pm \sigma}(\alpha, x, t, z)=W^{ \pm \sigma}(\alpha, x, t, \pm z) e^{ \pm x_{\sigma} z}
$$

Then (4.2) is equivalent to the following bilinear identity of the wave functions

$$
\begin{gathered}
\operatorname{Res}_{z=0} d z \Psi^{+0}(\alpha, x, t, z) S(z)^{t} \Psi^{-0}\left(\beta, x^{\prime}, t^{\prime}, z\right)= \\
\operatorname{Res}_{z=0} d z \Psi^{+1}(\alpha, x, t, z) S(z)^{t} \Psi^{-1}\left(\beta, x^{\prime}, t^{\prime}, z\right) .
\end{gathered}
$$

\section{Multicomponent BKP and DKP hierarchies; Sato and Lax equations}

We will now rewrite the bilinear identity for the wave functions in terms of formal pseudodifferential operators.

Let $N$ be some positive integer, $\partial=\frac{\partial}{\partial x}$, a formal $N \times N$ matrix pseudodifferential operator with parameters $t=\left(t_{j}^{(a)}\right)$ is an expression of the form

$$
P(x, t, \partial)=\sum_{j \in \mathbb{Z}} P_{j}(x, t) \partial^{j}
$$

where the $P_{j}$ 's are $N \times N$ matrices over the algebra of formal power series over $\mathbb{C}$ in the indeterminates $x$ and $t$. The largest $M$ such that $P_{M} \neq 0$ is called the order of $P(x, t, \partial)$ (we write $\operatorname{ord} P(x, t, \partial)=M$, we sometimes allow $M$ to be $\infty$ ). One defines the differential 
part of $P(x, t, \partial)$ by $P(x, t, \partial)_{+}=\sum_{j \geq 0} P_{j}(x, t) \partial^{j}$ and let $P_{-}=P-P_{+}$. The vector space $\Psi$ over $\mathbb{C}$ of all formal psuedodifferential operators decomposes in a natural way $\Psi=\Psi_{-} \oplus \Psi_{+}$and turns into a algebra; the product is defined by

$$
\partial^{k} \circ P(x, t) \partial^{\ell}=\sum_{j=0}^{\infty}\left(\begin{array}{c}
k \\
j
\end{array}\right) \frac{\partial^{j} P(x, t)}{\partial x^{j}} \partial^{k+\ell-j} .
$$

One has a natural linear anti-involution $*$ on $\Psi$, it is defined by

$$
\left(P(x, t) \partial^{\ell}\right)^{*}=(-\partial)^{\ell} \circ{ }^{t} P(x, t)
$$

It preserves the decomposition $\Psi=\Psi_{-} \oplus \Psi_{+}$.

The following Lemma will be very essential, for a proof see [11].

Lemma 5.1 Let $P(x, t, \partial)$ and $Q(x, t, \partial)$ be two formal pseudodifferential operators. Then

$$
\operatorname{Res}_{z=0} d z\left(P(x, t, \partial) e^{z x}\right)\left({ }^{t} Q\left(x^{\prime}, t^{\prime}, \partial^{\prime}\right) e^{-z x^{\prime}}\right)=\sum_{j=0}^{\infty} R_{j}\left(x, t, t^{\prime}\right) \frac{\left(x-x^{\prime}\right)^{j}}{j !}
$$

if and only if

$$
\left(P(x, t, \partial) \circ Q\left(x, t^{\prime}, \partial\right)^{*}\right)_{-}=\sum_{j=0}^{\infty} R_{j}\left(x, t, t^{\prime}\right) \partial^{-j-1}
$$

As a consequence of this lemma one has

Corollary 5.1 Let $P(x, t, \partial)$ and $Q(x, t, \partial)$ be two formal matrix pseudodifferential operators. Then

$$
\operatorname{Res}_{z=0} d z\left(P(x, t, \partial) e^{z x}\right)\left({ }^{t} Q\left(x^{\prime}, t^{\prime}, \partial^{\prime}\right) e^{-z x^{\prime}}\right)=\sum_{j} R_{j}(x, t) S_{j}\left(x^{\prime}, t^{\prime}\right)
$$

if and only if

$$
\left(P(x, t, \partial) Q\left(x, t^{\prime}, \partial\right)^{*}\right)_{-}=\sum_{j} R_{j}(x, t) \partial^{-1} S_{j}\left(x, t^{\prime}\right)
$$

Now let $\partial=\partial_{0}$ or $\partial_{1}$. Then

$$
\Psi^{ \pm \sigma}\left(\alpha, x_{0}, x_{1}, t, z\right)=W^{ \pm \sigma}\left(\alpha, x_{0}, x_{1}, t, \partial_{\sigma}\right) e^{ \pm x_{\sigma} z}
$$

where

$$
W^{ \pm \sigma}\left(\alpha, x_{0}, x_{1}, t, \partial_{\sigma}\right)=P^{ \pm \sigma}\left(\alpha, x_{0}, x_{1}, t, \partial_{\sigma}\right) Q^{ \pm \sigma}\left(t, \partial_{\sigma}\right) R^{ \pm \sigma}\left(t, \partial_{\sigma}\right) .
$$

Using the above corollary we deduce that (4.14) is equivalent to

$$
\begin{gathered}
\left(W^{+0}\left(\alpha, x_{0}, x_{1}, t, \partial_{0}\right) S\left(\partial_{0}\right) W^{-0}\left(\beta, x_{0}, x_{1}^{\prime}, t^{\prime}, \partial_{0}\right)^{*}\right)_{-} \\
=\operatorname{Res}_{z=0} d z W^{+1}\left(\alpha, x_{0}, x_{1}, t, z\right) S(z) e^{x_{1} z} \partial_{0}^{-1 t} W^{-1}\left(\beta, x_{0}, x_{1}^{\prime}, t^{\prime} z\right) e^{-x_{1}^{\prime} z}
\end{gathered}
$$

and

$$
\begin{gathered}
\left(W^{+1}\left(\alpha, x_{0}, x_{1}, t, \partial_{1}\right) S\left(\partial_{1}\right) W^{-1}\left(\beta, x_{0}^{\prime}, x_{1}, t^{\prime}, \partial_{1}\right)^{*}\right)_{-} \\
=\operatorname{Res}_{z=0} d z W^{+0}\left(\alpha, x_{0}, x_{1}, t, z\right) S(z) e^{x_{0} z} \partial_{1}^{-1}{ }^{-1} W^{-0}\left(\beta, x_{0}^{\prime}, x_{1}, t^{\prime}, z\right) e^{-x_{0} z}
\end{gathered}
$$


from which one deduces:

$$
\begin{aligned}
& \left(W^{+0}\left(\alpha, x_{0}, x_{1}, t, \partial_{o}\right) S\left(\partial_{0}\right) \partial_{1}^{-1} W^{-0}\left(\beta, x_{0}, x_{1}, t^{\prime}, \partial_{0}\right)^{*}\right)_{-} \\
& =\left(W^{+1}\left(\alpha, x_{0}, x_{1}, t, \partial_{1}\right) S\left(\partial_{1}\right) \partial_{0}^{-1} W^{-1}\left(\beta, x_{0}, x_{1}, t^{\prime}, \partial_{1}\right)^{*}\right)_{-} .
\end{aligned}
$$

Hence

$$
\begin{aligned}
& \left(W^{+0}\left(\alpha, x_{0}, x_{1}, t, \partial_{0}\right) S\left(\partial_{0}\right) W^{-0}\left(\beta, x_{0}, x_{1}, t^{\prime}, \partial_{0}\right)^{*}\right)_{-}= \\
& \operatorname{Res}_{\partial_{1}} W^{+1}\left(\alpha, x_{0}, x_{1}, t, \partial_{1}\right) S\left(\partial_{1}\right) \partial_{0}^{-1} W^{-1}\left(\beta, x_{0}, x_{1}, t^{\prime}, \partial^{\prime}\right)^{*}
\end{aligned}
$$

and

$$
\begin{aligned}
& \left(W^{+1}\left(\alpha, x_{0}, x_{1}, t, \partial_{1}\right) S\left(\partial_{1}\right) W^{-1}\left(, x_{0}, x_{1}, t^{\prime}, \partial_{1}\right)^{*}\right)_{-}= \\
& \operatorname{Res}_{\partial_{0}} W^{+0}\left(\alpha, x_{0}, x_{1}, t, \partial_{0}\right) S\left(\partial_{0}\right) \partial_{1}^{-1} W^{-0}\left(\beta, x_{0}, x_{1}, t^{\prime}, \partial_{0}\right)^{*}
\end{aligned}
$$

where

$$
\operatorname{Res} \partial \sum_{j \in \mathbb{Z}} P_{j}(x, t) \partial^{k}=P_{-1}(x, t) .
$$

Notice that

$$
\begin{aligned}
& Q^{ \pm \sigma}\left(t, \partial_{\sigma}\right)^{*}=Q^{\mp}\left(t, \partial_{\sigma}\right)^{-1}, \\
& R^{ \pm \sigma}\left(\alpha, \partial_{\sigma}\right)^{*}=R^{\mp \sigma}\left(\alpha, \partial_{\sigma}\right)^{-1}=R^{\mp}\left(-\alpha, \partial_{\sigma}\right) .
\end{aligned}
$$

Unfortunately, we cannot prove that $P^{ \pm \sigma}\left(\alpha, x_{0}, x_{1}, t, \partial_{\sigma}\right)$ is invertible for arbitrary $m$. Hence we do not know if it is possible to define the operator $L_{\sigma} \equiv L_{\sigma}\left(\alpha, x_{0}, x_{1}, t, \partial_{\sigma}\right)=$ $P^{+\sigma}\left(\alpha, x_{0}, x_{1}, t, \partial_{\sigma}\right) \partial_{\sigma} P^{+\sigma}\left(\alpha, x_{0}, x_{1}, t, \partial_{\sigma}\right)^{-1}$ such that

$$
L_{\sigma} \psi^{+\sigma}\left(\alpha, x_{0}, x_{1}, t, z\right)=z \psi^{+\sigma}\left(\alpha, x_{0}, x_{1}, t, z\right) .
$$

For that reason we assume from now on that $m=0$. It is then straightforward to check from the definition of $P^{ \pm \sigma}\left(\alpha, x_{0}, x_{1}, t, \partial_{\sigma}\right)$ (see (4.8)) that $P^{ \pm \sigma}\left(\alpha, x_{0}, x_{1}, t, \partial_{\sigma}\right)$ is indeed invertible, and that

$$
\begin{array}{ll}
P^{ \pm 1}\left(\alpha, x_{0}, x_{1}, t, \partial_{1}\right) & =2 I-P_{0}^{ \pm 0}\left(\alpha, x_{0}, x_{1}, t\right), \\
Q^{ \pm 1}\left(t, \partial_{1}\right)=R^{ \pm 1}\left(\alpha, \partial_{1}\right) & =I .
\end{array}
$$

So from now on we assume that $x_{0}=x_{1}$ and hence that there is only one $\partial_{\sigma}$, viz $\partial_{0}$, and write from now on $x$ and $\partial$ instead of $x_{0}$ and $\partial_{0}$.

We also remove all superscripts $\sigma$ and write $T^{ \pm}(\alpha, x, t)$ instead of $P^{ \pm 1}(\alpha, x, t, \partial)$. Then the bilinear identity of the wave function $\Psi^{ \pm}(\alpha, x, t, z)=W^{ \pm}(\alpha, x, t, \partial) e^{ \pm x z}$ takes the following form

$$
\begin{aligned}
& \operatorname{Res}_{z=0} d z \Psi^{+}(\alpha, x, t, z) S(z){ }^{t} \Psi\left(\beta, x^{\prime}, t^{\prime}, z\right) \\
& =\operatorname{Res}_{z=0} d z T^{+}(\alpha, x, t) S(z) e^{x z t} T^{-}\left(\alpha, x^{\prime}, t^{\prime}\right) e^{-x^{\prime} z} .
\end{aligned}
$$

Using Corollary 5.1 one deduces

$$
\begin{aligned}
& \left(W^{+}(\alpha, x, t, \partial) S(\partial) W^{-}\left(\beta, x, t^{\prime}, \partial\right)^{*}\right)_{-}= \\
& T^{+}(\alpha, x, t) \frac{1}{2} E_{00} \partial^{-1} T^{-}\left(\beta, x, t^{\prime}\right),
\end{aligned}
$$


from which we deduce, using (5.4 -5.5), that

$$
P^{-}(\alpha, x, t, \partial)^{*}=S(\partial)^{-1} P^{+}(\alpha, x, t, \partial)^{-1} T^{+}(\alpha, x, t) S(\partial){ }^{t} T^{-}(\alpha, x, t)
$$

and that

$$
\left(P^{+}(\alpha, x, t, \partial) R^{+}(\alpha-\beta, \partial) P^{+}(\beta, x, t, \partial)^{-1} T(\beta, x, t) S(\partial)\right)_{-}=\frac{1}{2} T(\alpha, x, t) E_{00} \partial^{-1} .
$$

In order to get nice formulas we change the wave function. Define

$$
\begin{aligned}
\Phi^{ \pm}(\alpha, x, t, z) & =V^{ \pm}(\alpha, x, t, \partial) e^{ \pm x z} \quad \text { where } \\
V^{ \pm}(\alpha, x, t, \partial) & =K^{ \pm}(\alpha, x, t, \partial) R^{ \pm}(\alpha, \partial) Q^{ \pm}(t, \partial) \quad \text { and } \\
K^{ \pm}(\alpha, x, t, \partial) & =T^{ \pm}(\alpha, x, t)^{-1} P^{ \pm}(\alpha, x, t, \partial) .
\end{aligned}
$$

Notice that

$$
\begin{aligned}
& \Phi^{ \pm}(\alpha, x, t, z)=P_{0}^{ \pm}(\alpha, x, t) \Psi^{ \pm}(\alpha, x, t, z), \\
& \Psi^{ \pm}(\alpha, x, t, z)=\left(\frac{3}{2} I-\frac{1}{2} K_{0}^{ \pm}(\alpha, x, t)\right) \Phi^{ \pm}(\alpha, x, t, z) .
\end{aligned}
$$

With the above definitions, formulas (5.6-5.9) turn into

$$
\begin{gathered}
\operatorname{Res}_{z=0} d z \Phi^{+}(\alpha, x, t, z) S(\partial){ }^{t} \Phi^{-}\left(\beta, x^{\prime}, t^{\prime}, z\right)=\frac{1}{2} E_{00} \\
\left(V^{+}(\alpha, x, t, \partial) S(\partial) V^{-}(\beta, x, t, \partial)\right)_{-}=\frac{1}{2} E_{00} \partial^{-1} \\
K^{-}(\alpha, x, t, \partial)^{*}=S(\partial)^{-1} K^{+}(\alpha, x, t, \partial)^{-1} S(\partial) \\
\left(K^{+}(\alpha, x, t, \partial) R^{+}(\alpha-\beta, \partial) K^{+}(\beta, x, t, \partial)^{-1} S(\partial)\right)_{-}=\frac{1}{2} E_{00} \partial^{-1} .
\end{gathered}
$$

To obtain Sato's equation we differentiate $(5.12)$ by $t_{\ell}^{(a)}$ and use lemma 5.1 and (5.14):

$$
\frac{\partial K^{+}(\alpha, x, t, \partial)}{\partial t_{\ell}^{(a)}}=-\left(K^{+}(\alpha, x, t, \partial) N(\ell, a) K^{+}(\alpha, x, t, \partial)^{-1} S(\partial)\right)_{-} S(\partial)^{-1} K^{+}(\alpha, x, t, \partial),
$$

where

$$
N(\ell, a))=\left\{\begin{array}{l}
\left.\partial^{\ell} E_{00} \quad \text { if } a=0 \text { (only if } \epsilon\left(n^{\prime}\right)=1\right), \\
\partial^{\ell} E_{a a}-(-\partial)^{\ell} E_{-a,-a} .
\end{array}\right.
$$

To make the formulas nicer, we introduce a new decomposition of $\Psi$, viz, let

$$
P_{\geq}=(P S(\partial))_{+} S(\partial)^{-1}, P_{<}=P-P_{\geq}
$$

then

$$
\Psi=\Psi_{<} \oplus \Psi_{\geq}
$$


and $(5.15)$ and (5.16) turn into

$$
\begin{gathered}
\left(K^{+}(\alpha, x, t, \partial) R^{+}(\alpha-\beta, \partial) K^{+}(\beta, x, t, \partial)^{-1}\right)_{<}=E_{00}, \\
\frac{\partial K^{+}(\alpha, x, t, \partial)}{\partial t_{\ell}^{(a)}}=-\left(K^{+}(\alpha, x, t, \partial) N(\ell, a) K^{+}(\alpha, x, t, \partial)^{-1}\right)_{<} K^{+}(\alpha, x, t, \partial) .
\end{gathered}
$$

This new decomposition of $\Psi$ is very natural, it is based on the following observations.

Lemma 5.2 Let $A=\sum_{j=-n}^{n} E_{j,-j}$, then

$$
K^{+}(\alpha, x, t, \partial)=A K^{-}(\alpha, x, t, \partial) A
$$

The proof of this lemma is a straightforward verification of the following two facts $T^{+}(\alpha, x, t, \partial)=A T^{-}(\alpha, x, t, \partial) A$ and $P^{+}(\alpha, x, t, \partial)=A P^{-}(\alpha, x, t, \partial) A$.

Let $D=S(\partial) A$, then

$$
D=\frac{1}{2} E_{00} \partial^{-1}+\sum_{j=1}^{n}\left(E_{j,-j}-E_{-j, j}\right)
$$

and define the linear anti involution $i: \Psi \rightarrow \Psi$ by

$$
i(P(x, t, \partial))=D P(x, t, \partial)^{*} D^{-1}
$$

Then $i$ preserves the decomposition $\Psi=\Psi_{<} \oplus \Psi_{\geq}$and

$$
\begin{aligned}
& i\left(K^{+}(\alpha, x, t, \partial)\right)=K^{+}(\alpha, x, t, \partial)^{-1}, \\
& i\left(Q^{+}(t, \partial)\right)=Q^{+}(t, \partial)^{-1}, \\
& i\left(R^{+}(\alpha, \partial)\right)=R^{+}(\alpha, \partial)^{-1}, \\
& i\left(V^{+}(\alpha, x, t, \partial)\right)=V^{+}(\alpha, x, t, \partial)^{-1}, \\
& i\left(K^{+}(\alpha, x, t, \partial) N(\ell, a) K^{+}(\alpha, x, t, \partial)^{-1}\right)=-K^{+}(\alpha, x, t, \partial) N(\ell, a) K^{+}(\alpha, x, t, \partial)^{-1} .
\end{aligned}
$$

The last line of (5.22) is a consequence of the following

Lemma 5.3 Let $P(x, t \partial)$ be a pseudodifferential operator then

$$
i\left(V^{+}(\alpha, x, t, \partial) P(x, t, \partial) V^{+}(\alpha, x, t, \partial)^{-1}=\lambda V^{+}(\alpha, x, t, \partial) P(x, t, \partial) V^{+}(\alpha, x, t, \partial)^{-1}\right.
$$

if and only if $i(P(x, t, \partial))=\lambda P(x, t, \partial)$.

Notice that the subspace

$$
\Psi^{i}=\{P \in \Psi \mid i(P)=-P\}
$$

is a subalgebra of $\Psi$ with respect to the product $[A, B]=A \circ B-B \circ A$, it will play an important role in the theorey of the BKP hierarchy.

Proposition 5.1 Let $\alpha, \beta \in$ supp $\tau$ such that $\alpha-\beta=\delta_{i}$ or $-\delta_{i}$. Then $K(\alpha) \equiv$ $K^{+}(\alpha, x, t, \partial)$ is completely determined by $K(\beta) \equiv K^{+}(\beta, x, t, \partial)$. 


\section{Proof.}

For simplicity of notations we only consider the case that $\alpha-\beta=\delta_{i}$. Then $R^{+}(\alpha-\beta)=$ $A \partial+B+C \partial^{-1}$ where

$$
A=E_{i i}, \quad B=-E_{00}+\sum_{j=1, j \neq i}^{n} \epsilon_{j i} E_{j j}, \quad C=-E_{-i,-i} .
$$

For $K(\alpha)=\sum_{j=0}^{\infty} K(\alpha)^{(j)}=K(\alpha)^{(0)}\left(I+\sum_{j=1}^{\infty} W(\alpha)^{(j)}\right)$, its inverse is

$$
\begin{aligned}
K(\alpha)^{-1} & =\left(I-W(\alpha)^{(1)}+\ldots\right) K(\alpha)^{(0)^{-1}} \\
& =\left(I-K(\alpha)^{(0)^{-1}} K(\alpha)^{(1)}+\ldots\right) K(\alpha)^{(0)^{-1}}
\end{aligned}
$$

It follows from $(5.18)$ that

$$
\left(K(\alpha)\left(A \partial+B+C \partial^{-1}\right) K(\beta)^{-1}\right)_{<}=E_{00} .
$$

Now calculating $\left(K(\alpha)\left(A \partial+B+C \partial^{-1}\right) K(\beta)^{-1}\right) \geq$ we obtain

$$
\begin{aligned}
& K(\alpha)\left(A \partial+B+C \partial^{-1}\right) K(\beta)^{-1}= \\
& K(\alpha)^{(0)} A\left[K(\beta)^{(0)^{-1}} \partial+\partial\left(K(\beta)^{(0)^{-1}}\right)-W(\beta)^{(1)} K(\beta)^{(0)^{-1}}\right]+K(\alpha)^{(1)} A K(\beta)^{(0)^{-1}}+ \\
& K(\alpha)^{(0)} B K(\beta)^{(0)^{-1}}-\left(K(\alpha)^{(0)} A\left[\partial\left(K(\beta)^{(0)^{-1}}\right)-W(\beta)^{(1)} K(\beta)^{(0)^{-1}}\right]\right. \\
& \left.+K(\alpha)^{(1)} A K(\beta)^{(0)^{-1}}+K(\alpha)^{(0)} B K(\beta)^{(0)^{-1}}-1\right) E_{00} .
\end{aligned}
$$

Now multiply this from the left by $K(\alpha)^{(0)^{-1}}$ and from the right by $K(\beta)$ and equating the coefficients of $\partial^{m}$ we find for $\partial^{0}$ :

$$
\begin{aligned}
& \left\{A\left[\partial\left(K(\beta)^{(0)^{-1}}\right)-W(\beta)^{(1)} K(\beta)^{(0)^{-1}}\right]+W^{(1)}(\alpha) A K(\beta)^{(0)^{-1}}+\right. \\
& \left.B K(\beta)^{(0)^{-1}}-K(\alpha)^{(0)^{-1}}\right\} E_{00} K(\beta)^{(0)}=0
\end{aligned}
$$

and for $\partial^{-j}, \quad j=1,2, \ldots$ :

$$
\begin{aligned}
& W(\alpha)^{(j+1)} A+W(\alpha)^{(j)} B+W(\alpha)^{(j-1)} C= \\
& A\left[\partial\left(W(\beta)^{(j)}\right)+W(\beta)^{(j+1)}-W(\beta)^{(1)} W(\beta)^{(j)}\right]+W(\alpha)^{(1)} A W(\beta)^{(j)}+ \\
& B W(\beta)^{(j)}-\left\{A\left[\partial\left(K(\beta)^{(0)^{-1}}\right)-W(\beta)^{(0)} K(\beta)^{(0)^{-1}}\right]+W(\alpha)^{(1)} A K(\beta)^{(0)^{-1}}\right. \\
& \left.+B K(\beta)^{(0)^{-1}}-K(\alpha)^{(0)^{-1}}\right\} E_{00} K(\beta)^{(j)} .
\end{aligned}
$$

From (5.23) we deduce that

$$
\begin{aligned}
& \left\{A\left[\partial\left(K(\beta)^{(0)^{-1}}\right) W(\beta)^{(1)} K(\beta)^{(0)^{-1}}\right]+W(\alpha)^{(1)} A K(\beta)^{(0)^{-1}}+\right. \\
& \left.B K(\beta)^{(0)^{-1}}-K(\alpha)^{(0)^{-1}}\right\} E_{00}=0 .
\end{aligned}
$$

Now substitute this in (5.24) and one obtains

$$
\begin{aligned}
& W(\alpha)^{(j+1)} A+W(\alpha)^{(j)} B+W(\alpha)^{(j-1)} C= \\
& A\left[\partial\left(W(\beta)^{(j)}\right)+W(\beta)^{(j+1)}-W(\beta)^{(1)} W(\beta)^{(j)}\right]+W(\alpha)^{(1)} A W(\beta)^{(j)}+B W(\beta)^{(j)}\left({ }^{(5.26)}\right.
\end{aligned}
$$


It is clear from $(5.26)$ that alle $W(\alpha)^{(j)}$, except $\left(W(\alpha)_{\ell i}^{(1)}\right.$, can be expressed in $W(\beta)$ and $\left(W(\alpha)^{(1)}\right)_{\ell, i}(-n \leq \ell \leq n)$.

Now take the $(0,0)$-entry of (5.25) one has

$$
\left(W(\alpha)^{(1)}\right)_{0 i}\left(K(\beta)^{(0)^{-1}}\right)_{i 0}=2 .
$$

Now notice that

$$
\begin{aligned}
& \left(K(\gamma)^{(0)^{-1}}-I\right)=-\left(K(\gamma)^{(0)}-I\right)=-2\left(P(\gamma)^{(0)}-I\right), \\
& \left(P(\gamma)^{(0)}-I\right)_{j k}=0 \quad \text { for } k \neq 0 \quad \text { and } j=k=0 .
\end{aligned}
$$

Using this we take the $(j, 0)$-th entry of $(5.25)$ and deduce from this that $\left(W(\alpha)^{(1)}\right)_{0 i}$ can be expressed in $K(\beta)^{(0)}, W(\beta)^{(1)}$ and $K(\alpha)^{(0)}$. Hence using (5.27) it suffices to determine $\left(P(\alpha)^{(0)}\right)_{j 0} \quad(j \neq 0)$.

Now for $j \neq 0$ one has:

$$
\left(P(\alpha)^{(0)}\right)_{j 0}=\left\{\begin{array}{cl}
-\frac{\tau_{\beta+\delta_{i}-\delta_{j}}}{\tau_{\beta+\delta_{i}}} & j>0 \\
-\frac{\tau_{\beta+\delta_{i}+\underline{\varepsilon}_{j}}}{\tau_{\beta+\delta_{i}}} & j<0 .
\end{array}\right.
$$

Since $\left(P(\beta)^{(0)}\right)_{i 0}=-\tau_{\beta+\delta_{i}} / \tau_{\beta} \neq 0$ one deduces from $(4.8)$ that

$$
\left(P(\alpha)^{(0)}\right)_{j 0}= \begin{cases}\epsilon_{i j}\left[\left(P(\beta)^{(0)}\right)_{i 0}\right]^{-1}\left(P(\beta)^{\left(1-\delta_{i j}\right)}\right)_{i j} & j>0 \\ -\epsilon_{i,-j}\left[\left(P(\beta)^{0)}\right)_{i 0}\right]^{-1}\left(P(\beta)^{\left(1+\delta_{i,-j}\right)}\right)_{i j} & j<0\end{cases}
$$

Hence $K(\alpha)$ is completely determined by $K(\beta)$.

From now on we write $V(\alpha), K(\alpha), R(\alpha)$, etc. instead of $V^{+}(\alpha, x, t, \partial), K^{+}(\alpha, x, t, \partial)$, etc. Define the following operators:

$$
\begin{array}{ll}
L(\alpha) & =V(a) \partial^{-1} V(\alpha)^{-1}, \\
M(\alpha) & =V(\alpha) x V(\alpha)^{-1}, \\
N(\alpha, \beta) & =V(\alpha) V(\beta)^{-1}, \\
C_{i j}(\alpha) & =V(\alpha) E_{i j} V(\alpha)^{-1} .
\end{array}
$$

where we write $x$ and $\partial$ for $x I_{2 n+1}$ and $\partial I_{2 n+1}$.

Notice, that several of the products appearing in (5.28) consist of products of infinite order pseudodifferential operators. Mulase showed in 12 that being careful enough, one can indeed take products of infinite order operators. For more details see [12], [13]. The above operators act on the wave function, as follows

$$
\begin{array}{ll}
L(\alpha) \Phi(\alpha, z) & =\Phi(\alpha, z) z, \\
M(\alpha) \Phi(\alpha, z) & =\Phi(\alpha, z) x, \\
N(\alpha, \beta) \Phi(\beta, z) & =\Phi(\alpha, z), \\
C_{i j}(\alpha) \Phi(\alpha, z) & =\Phi(\alpha, z) E_{i j} .
\end{array}
$$


It is easy to see that the operators $L(\alpha), N(\alpha, \beta)$ and $C_{i}(\alpha):=C_{i i}(\alpha)$ are well-defined, they are finite order formal pseudodifferential operators:

$$
\begin{aligned}
& L(\alpha)=K(\alpha) \partial K(\alpha)^{-1}, \\
& N(\alpha, \beta)=K(\alpha) R(\alpha-\beta) K(\beta)^{-1}, \\
& C_{i}(\alpha)=K(\alpha) E_{i i} K(\alpha)^{-1} .
\end{aligned}
$$

Remark 5.1 It is important to notice that the operators $L(\alpha), M(\alpha)$ and $C_{i j}(\alpha)$ have the same commutation relations as $\partial, x$ and $E_{i j}$ respectively.

We deduce from (5.18 5.19) that

$$
\begin{aligned}
& N(\alpha, \beta)=N(\alpha, \beta)_{\geq}+E_{00}, \\
& \frac{\partial V(\alpha)}{\partial t_{\ell}^{(a)}}=B(\alpha, \ell, a) V(\alpha),
\end{aligned}
$$

where

$$
B(\alpha, \ell, a)=\left(K(\alpha) N(\ell, a) K(\alpha)^{-1}\right)_{\geq}
$$

This leads to

$$
\begin{aligned}
\frac{\partial L(\alpha)}{\partial t_{\ell}^{(a)}} & =[B(\alpha, \ell, a), L(\alpha)], \\
\frac{\partial M(\alpha)}{\partial t_{\ell}^{(a)}} & =[B(\alpha, \ell, a), M(\alpha)], \\
\frac{\partial N^{(\alpha, \beta)}}{\partial t_{\ell}^{(a)}} & =B(\alpha, \ell, a) N(\alpha, \beta)-N(\alpha, \beta) B(\beta, \ell, a), \\
\frac{\partial C_{i j}(\alpha)}{\partial t_{\ell}^{(a)}} & =\left[B(\alpha, \ell, a), C_{i j}(\alpha)\right] .
\end{aligned}
$$

and to

$$
\frac{\partial \Phi(\alpha, z)}{\partial t_{\ell}^{(a)}}=B(\alpha, \ell, a) \Phi(\alpha, z) .
$$

The equations (5.33) are called Lax equations, they are a direct consequence of the Sato equation.

Let $\epsilon\left(n^{\prime}\right)=0$ in this case a reduction to the DKP hierarchy and KP hierarchy is possible. Notice that a BKP tau function $\tau=\sum_{\alpha} \tau_{\alpha} e^{\alpha}$ is a DKP (resp. KP) tau function if $\operatorname{supp} \tau \subset$ $\mathfrak{N}_{\overline{0}}$ or $\operatorname{supp} \tau \subset \mathfrak{N}_{\overline{1}}$ (resp. $\operatorname{supp} \tau \subset \mathfrak{M}_{k}$ for some $k \in \mathbb{Z}$ ). The corresponding wave functions $\Phi(\alpha, z)$ then have a simpler form, in fact $\Phi(\alpha, z)=\Psi(\alpha, z)$ i.e., $\Psi(\alpha, z)_{i 0}=\Psi(\alpha, z)_{0 i}=0$ for $i \neq 0$. If $\tau$ is a KP tau function and $\alpha \in \operatorname{supp} \tau$, then $\Phi(\alpha, z)$ has an even simpler form. One finds that also $\Psi(\alpha, z)_{i j}=0$ for $i>0>j$ and $i<0<j$. And the corresponding wave function is of the form

$$
\left(\begin{array}{ccc} 
& 0 & \\
\Psi^{-}(\alpha,-z) & \vdots & 0 \\
& 0 & \\
0 \ldots 0 & 1 & 0 \ldots 0 \\
& 0 & \\
0 & \vdots & \Psi^{+}(\alpha, z) \\
& 0 &
\end{array}\right)
$$


Here $\Psi^{+}(\alpha, z)$ is a wave function of the $n$-component KP hierarchy (see 11]) and $\Psi^{-}(\alpha, z)$ is up to permutation of the indices the adjoint wave function.

\section{The Orlov-Schulman-Adler-Shiota-van Moerbeke formula}

In this section we will derive a generalization of the Orlov-Schulman-Adler-Shiota-van Moerbeke (OSASM) formula for the BKP hierarchy. A formula that connects the action of some $W$-algebra on the tau functions to the so-called additional symmetries on the wave function. This formula was stated for the (1-component) KP hierarchy by Orlov and Schulman [14] and proved by Adler, Shiota and van Moerbeke ( [1], [2] see also [19]). Operators from the subspace $\Psi^{i}=\{P \in \Psi \mid i(P)=-P\}$ of the space of formal pseudodifferential operators, which form a subalgebra with respect to the Lie product $[A, B]=A \circ B-B \circ A$, appear in this OSASM-formula.

Notice that the subspace generated by $L, L^{-1}, M$ and $C_{i j}$ is isomorphic to $D\left(g l_{2 n+1}\right)$, the algebra of differential operators on $\mathbb{C}\left[t, t^{-1}\right]^{2 n+1}$. The intersection $D\left(g l_{2 n+1}\right) \cap \Psi^{i}$ then forms a subalgebra $D^{(2)}\left(g l_{2 n+1}\right)$ of $D\left(g l_{2 n+1}\right)$. From now on let $\epsilon=\epsilon\left(n^{\prime}\right)=0$ if $n^{\prime}=0$ and $=1$ if $n^{\prime}=1$. If $\epsilon=0$ we need yet another subalgebra, viz $D^{(2)}\left(g l_{2 n}\right)$. We view this algebra as the subalgebra of $D^{(2)}\left(g l_{2 n+1}\right)$ where the 0 th row and column are zero. A central extension $W_{1+\infty}^{(2)}\left(g l_{2 n+\epsilon}\right)=D^{(2)}\left(g l_{2 n+\epsilon}\right) \oplus \mathbb{C} c$ of $D^{(2)}\left(g l_{2 n+\epsilon}\right)$ can also be formulated in terms of the fermions.

Let $p, q \in\{0,1,2, \ldots, n\}, \nu, \sigma \in\{+,-\}$ and define

$$
X^{\nu p, \sigma q}(y, w)=: \psi^{\nu p}(\nu y) \frac{\psi^{\sigma q}(-\sigma w)}{w^{\delta_{q}, 0}}:,
$$

where $p, q \neq 0$ if $\epsilon=0$ and if $\epsilon=1$ and $p=0$ or $q=0$ then we assume that $\nu=+, \sigma=+$ respectively. Notice that

$$
X^{\nu p, \sigma q}(y, w)=\psi^{\nu p}(\nu y) \frac{\psi^{\sigma q}(-\sigma w)}{w^{\delta_{q}, 0}}-\frac{\delta_{p q} \nu}{y-w}\left(\delta_{p, 0}+\left(1-\delta_{p, 0}\right) \delta_{\nu,-\sigma}\right) .
$$

We write

$$
\begin{aligned}
X^{\nu p, \sigma q}(y, w) & =\sum_{\ell=0}^{\infty} \frac{(y-w)^{\ell}}{\ell !} X^{\ell+1, \nu p, \sigma q}(w), \\
X^{\ell+1, \nu p, \sigma q}(w) & =: \frac{\partial^{\ell} \psi^{\nu p}(\nu w)}{\partial w^{\ell}} \frac{\psi^{\sigma q}(-\sigma w)}{w^{\delta_{q 0}}}: \\
& =\sum_{k \in \mathbb{Z}} X_{k}^{\ell+1, \nu p, \sigma q} w^{-k-\ell-1} .
\end{aligned}
$$

We can now calculate the Lie bracket of the elements $X_{k}^{\ell+1, \nu p, \sigma q}$ and $X_{m}^{n+1, \mu r, \lambda s}$, for that purpose we use the folowing. The operator $X_{k}^{\ell+1, \nu p, \sigma q}$ acts on the Clifford algebra by derivations via the adjoint representation $(a \in C \ell V)$ :

$$
X_{k}^{\ell+1, \nu p, \sigma q}(a)=\left[X_{k}^{\ell+1, \nu p, \sigma q}, a\right] .
$$

By identifying $(j \in \mathbb{Z}, \nu=+,-)$ :

$$
\begin{aligned}
\nu^{j} \psi_{j+\frac{1}{2}}^{-\nu p} & =t^{j} e_{\nu p}, \\
(-)^{j} \psi_{j}^{0} & =\sqrt{2} t^{j} e_{0} \quad \text { if } \epsilon=1,
\end{aligned}
$$


where $e_{j},-n \leq j \leq n$, is a basis of $\mathbb{C}^{2 n+\epsilon}$ (we assume that $j \neq 0$ if $\epsilon=0$ ), we can identify, via (6.4), the elements $X_{n}^{\ell+1, \nu p, \sigma q}$ with certain elements in $W_{1+\infty}^{(2)}\left(g l_{2 n+\epsilon}\right)$. One easily verifies that

$$
\begin{aligned}
X_{k}^{\ell+1, \nu p, \sigma q}= & \sum_{j \in \mathbb{Z}}[j]_{\ell} \nu^{j}(-\sigma)^{-j-k-1+\delta_{q 0}}: \psi_{-j-\frac{1-\delta_{p 0}}{2}}^{\nu p} \psi_{j+k+\frac{1-\delta_{q 0}}{2}}^{\sigma q}: \\
= & -(-\sigma)^{1-\delta_{q 0}} s(q, p) t^{k+\ell}\left(\frac{\partial}{\partial t}\right)^{\ell} E_{-\sigma q, \nu p} \\
& -(-\nu)^{1-\delta_{p 0}} s(p, q) t^{\delta_{p 0}}\left(\frac{\partial}{\partial t}\right)^{\ell}(-t)^{\ell+k-\delta_{q 0}} E_{-\nu p, \sigma q},
\end{aligned}
$$

where $[j]_{\ell}=j(j-1) \ldots(j-\ell+1)$ and $s(p, q)=(\sqrt{2})^{\delta_{p 0}-\delta_{q 0}}$.

Using Wicks theorem we can calculate the commutation relations between $X_{k}^{\ell+1, \nu p, \sigma q}$ and $X_{m}^{\ell+1, \mu r, \lambda s}$. One finds that one has a central extension:

$$
\begin{aligned}
& {\left[X_{k}^{\ell+1, \vee p, \sigma q}, X_{m}^{n+1, \mu r, \lambda s}\right]=} \\
& \quad X_{k}^{\ell+1, \nu p, \sigma q} X_{m}^{n+1, \mu r, \lambda s}-X_{m}^{n+1, \mu r, \lambda s} X_{k}^{n+1, \nu p, \sigma q}+\alpha\left(X_{k}^{\ell+1, \nu p, \sigma q}, X_{m}^{n+1, \mu p, \lambda s}\right) .
\end{aligned}
$$

The first part of the left-hand-side of $(6.6)$ can be determined using (6.5). The two cocycle $\alpha$ is determined as follows. The constant term of the OPE

$$
: \psi^{\nu p}(\nu y) \frac{\psi^{\sigma q}(-\sigma w)}{w^{\delta, 0}}:: \psi^{\mu r}(\mu u) \frac{\psi^{\lambda s}(-\lambda v)}{v^{\delta s, 0}}: \text {, }
$$

where we assume that $\nu, \sigma, \mu, \lambda=+$ whenever $p, q, r, s=0$ respectively, is equal to

$$
\begin{aligned}
& \frac{\nu \delta_{p s}}{y-v}\left(\delta_{p 0}+\left(1-\delta_{p 0}\right) \delta_{\nu,-\lambda}\right) \frac{\mu \delta_{q r}}{w-u}\left(\delta_{q 0}+\left(1-\delta_{q 0}\right) \delta_{\sigma,-\mu}\right) \\
& -\frac{\nu \delta_{p r}}{y+u}\left(-\delta_{p 0} u+\left(1-\delta_{p 0}\right) \delta_{\nu,-\mu}\right) \frac{\lambda \delta_{q s}}{w+v}\left(\frac{\delta_{q 0}}{v}+\left(1-\delta_{q 0}\right) \delta_{\sigma,-\lambda}\right) .
\end{aligned}
$$

Now differentiate this $\ell$ times to $y$ and $n$ times to $u$ and put $w=y$ and $v=u$, we thus find

$$
\begin{aligned}
& \delta_{p s} \delta_{q r} \nu \mu\left(\delta_{p 0}+\left(1-\delta_{p 0}\right) \delta_{\nu,-\lambda}\right)\left(\delta_{q 0}+\left(1-\delta_{q 0}\right) \delta_{\sigma,-\mu}\right) \frac{(-)^{\ell} \ell ! n !}{(y-u)^{\ell+n+2}} \\
& -\delta_{p r} \delta_{q s} \nu \lambda\left(\frac{\delta_{q 0}}{u}+\left(1-\delta_{q 0}\right) \delta_{\sigma,-\lambda}\right) \times \\
& \left(\left(-\delta_{p 0} u+\left(1-\delta_{p 0}\right) \delta_{\nu,-\mu}\right) \frac{(-)^{\ell+n}(\ell+n) !}{(y+u)^{\ell+n+2}}-\frac{\delta_{p 0} n(-)^{\ell+n-1}(\ell+n-1) !}{(y+u)^{\ell+n+1}}\right) .
\end{aligned}
$$

From which we conclude that

$$
\begin{aligned}
& \alpha\left(X_{k}^{\ell+1, \nu p, \sigma q}, X_{m}^{n+1, \mu r, \lambda s}\right)= \\
& \delta_{p s} \delta_{q r} \nu \mu\left(\delta_{p 0}+\left(1-\delta_{p 0}\right) \delta_{\nu,-\lambda}\right)\left(\delta_{q 0}+\left(1-\delta_{q 0}\right) \delta_{\sigma,-\mu}\right)(-)^{\ell} \ell ! n !\left(\begin{array}{c}
k+\ell \\
\ell+n+1
\end{array}\right) \delta_{k,-m} \\
& -\delta_{p r} \delta_{q s} \nu \lambda\left(\delta_{q 0}+\left(1-\delta_{q 0}\right) \delta_{\sigma,-\lambda}\right)\left\{-\delta_{p 0}(\ell+n+1) !\left(\begin{array}{c}
k+\ell+1 \\
\ell+n+1
\end{array}\right)+\right. \\
& \left.\left(1-\delta_{p 0}\right) \delta_{\nu, \mu}(\ell+n) !\left(\begin{array}{c}
k+\ell \\
\ell+n+1
\end{array}\right)\right\}(-)^{k+\ell+1} \delta_{k,-m-\delta_{p 0}+\delta_{q 0}}
\end{aligned}
$$

In a similar way as for pseudodifferential operators we have a linear anti-involution $*$ on $D\left(g l_{2 n+1}\right)$, let $A \subset g l_{2 n+1}$ then we define

$$
\left(t^{k}\left(\frac{\partial}{\partial t}\right)^{\ell} A\right)^{*}=\left(\frac{\partial}{\partial t}\right)^{\ell}(-t)^{k}{ }^{t} A .
$$


Let (compare with $D$, see 5.20 )

$$
C=\frac{1}{2} E_{00} t^{-1}+\sum_{j=1}^{n} E_{j,-j}-E_{-j, j},
$$

then we also have a linear anti-involution on $D\left(g l_{2 n+1}\right)$ that is compatible with $i$ on $\Psi$, for notational convenience we shall denote this involution also by $i$; let $X \in D\left(g l_{2 n+1}\right)$ then

$$
i(X)=C^{-1} X^{*} C .
$$

We can extend $i$ to $W_{1+\infty}\left(g l_{2 n+1}\right)$, by defining $i(c)=-c$. Now notice that the elements (6.5) satisfy

$$
i\left(X_{k}^{\ell+1, \nu p, \sigma q}\right)=-X_{k}^{\ell+1, \nu p, \sigma q} .
$$

Let $\alpha \in \mathcal{L}$, in analogy with (6.3) and (6.5) we define

$$
\begin{aligned}
Y^{\nu p, \sigma q}(\alpha, y, w)= & \sum_{\ell=0}^{\infty} \sum_{k \in \mathbb{Z}} \frac{(y-w)^{\ell}}{\ell !} w^{-k-\ell-1}\left\{(-\sigma)^{1-\delta_{q 0}} s(q, p) M(\alpha)^{\ell} L(\alpha)^{k+\ell} C_{\nu p,-\sigma q}(\alpha)+\right. \\
& \left.(-\nu)^{1-\delta_{p 0}} s(p, q)(-L(\alpha))^{k+\ell-\delta_{q 0}} M(\alpha)^{\ell} L(\alpha)^{\delta_{p 0}} C_{\sigma q,-\nu p}(\alpha)\right\}, \\
Z^{\nu p, \sigma q}(\alpha, y, w)= & P^{(0)-1}(\alpha) Y^{\nu p, \sigma q}(\alpha, y, w) P^{(0)-1}(\alpha) .
\end{aligned}
$$

Then

$$
\begin{aligned}
& Y^{\nu p, \sigma q}(\alpha, y, w) \Psi(\alpha, z)= \\
= & \sum_{\ell=0}^{\infty} \sum_{k \in \mathbb{Z}} \frac{(y-w)^{\ell}}{\ell !} w^{-k-\ell-1}\left\{(-\sigma)^{1-\delta_{q 0}} s(q, p) z^{k+\ell}\left(\frac{\partial}{\partial z}\right)^{\ell} \Psi(\alpha, z) E_{\nu p,-\sigma q}+\right. \\
& \left.(-\nu)^{1-\delta_{p 0}} s(p, q) z^{\delta_{p 0}}\left(\frac{\partial}{\partial z}\right)^{\ell}(-z)^{k+\ell-\delta_{q 0}} \Psi(\alpha, z) E_{\sigma q,-\nu p}\right\} \\
= & (-\sigma)^{1-\delta_{q 0}} s(q, p) \delta(w-z) \Psi(\alpha, y) E_{\nu p,-\sigma q}+ \\
& (-\nu)^{1-\delta_{p 0}} s(p, q) z^{\delta_{p 0}} e^{(y-w) \frac{\partial}{\partial z}} w^{-\delta_{q 0}} \delta(w+z) \Psi(\alpha,-w) E_{\sigma q,-\nu p} \\
= & (-\sigma)^{1-\delta_{q 0}} s(q, p) \delta(w-z) \Psi(\alpha, y) E_{\nu p,-\sigma q}+ \\
& (-\nu)^{1-\delta_{p 0}} s(p, q)(-y)^{\delta_{p 0}} w^{-\delta_{q 0}} \delta(y+z) \Psi(\alpha,-w) E_{\sigma q,-\nu p} .
\end{aligned}
$$

We have now given all the ingredients to determine the OSASM-formula. The proof of this formula is based on the following simple observation. Let $v \in V$ then

$$
(v \otimes 1) S=1 \otimes v-S(v \otimes 1) .
$$

From (6.14) we deduce that $(u, v \in V)$

$$
S(u v \tau \otimes \tau)+u \tau \otimes v \tau-v \tau \otimes u \tau=\frac{1}{2} u v \tau \otimes \tau .
$$

Now replacing $u$ by $\psi^{\nu p}(\nu y)$ and $v$ by $\psi^{\sigma q} \frac{(-\sigma w)}{w^{\delta q 0}}$ the equation (6.15) still holds. Next using (6.2) we obtain

$$
\begin{aligned}
& S^{\nu p, \sigma q}(y, w) \tau \otimes \tau+\psi^{\nu p}(\nu y) \tau \otimes \frac{\psi^{\sigma q}(-\sigma w)}{w^{\delta_{q 0}}}-\frac{\psi^{\sigma q}(-\sigma w)}{w^{\delta_{q 0}}} \tau \otimes \psi^{\nu p}(\nu y) \tau= \\
& \frac{1}{2} X^{\nu p, \sigma q}(y, w) \tau \otimes \tau .
\end{aligned}
$$


Again we assume that $\nu, \sigma=+$ whenever $p$, respectively $q=0$. In the same way as in section 4 we use the vertex operators for these fermionic fields and replace all $t_{1}^{(a)}$ by $t_{1}^{(a)}+x$. We then take the coefficient of

$$
e^{\alpha+\lambda \delta_{j}} \otimes e^{\beta-\mu \delta_{k}}, \quad \lambda, \mu=+,-, 0 \leq j, k \leq n
$$

From now on we assume that $\delta_{0}=0$ whenever $\epsilon=0$. Since $X^{\nu p, \sigma q}(y, w)$ is not well-defined on $\tau_{\gamma}$ we define

$$
\begin{gathered}
\mathbb{X}^{\nu p}(z) \tau_{\gamma}(x, t)=\left(\left(1-\delta_{p, 0}\right) \epsilon\left(\delta_{p}, \gamma\right) z^{\left(\nu \delta_{p} \mid \gamma\right)}+\delta_{p, 0} \frac{(-)^{(\delta \mid \gamma)}}{\sqrt{2}} e^{\nu \xi_{p}(t, z)} e^{-\nu \eta_{p}(t, z)} t_{\gamma}(x, t) e^{\nu z x}\right. \\
\mathbb{X}^{\nu p, \sigma q}(y, w) \tau_{\gamma}(x, t)=\left(\left(1-\delta_{p, 0}\right) \epsilon\left(\delta_{p}, \gamma+\delta_{q}\right)(\nu y)^{\left(\nu d_{p} \mid \gamma+\sigma \delta_{q}\right)}+\delta_{p, 0} \frac{(-)^{\left(\delta \mid \gamma+\sigma \delta_{q}\right)}}{\sqrt{2}}\right) \times \\
e^{\nu \xi_{p}(t, \nu y)} e^{-\nu \eta_{p}(t, \nu y)} \frac{\mathbb{X}^{\sigma q}(-\sigma w)}{w^{\delta_{q 0}}} \tau_{\gamma}(x, t) e^{y x}
\end{gathered}
$$

Using all this (6.16) turns into

$$
\begin{gathered}
\operatorname{Res}_{z=0} d z\left\{\frac{1-\epsilon}{2 z}(-)^{\left(\delta \mid \alpha+\lambda \delta_{j}\right)} \mathbb{X}^{\nu p, \sigma q}(y, w) \tau_{\alpha+\lambda \delta_{j}-\nu \delta_{p}-\sigma \delta_{q}}(x, t) e^{x z} \otimes(-)^{\left(\delta \mid \beta-\mu \delta_{k}\right)} \tau_{\beta-\mu \delta_{k}}(x, t) e^{-x z}\right. \\
+\frac{\epsilon(-)^{\left(\delta \mid \alpha+\lambda \delta_{j}\right)}}{\sqrt{2}} e^{\xi_{0}(t, z)} e^{-\eta_{0}(t, z)} \mathbb{X}^{\nu p, \sigma q}(y, w) \tau_{\alpha+\lambda \delta_{j}-\nu \delta_{p}-\sigma \delta_{q}}(x, t) e^{x z} \otimes \frac{\mathbb{X}^{0}(-z)}{z} \tau_{\beta-\mu \delta_{k}} \\
+\sum_{a=1}^{n} \epsilon\left(\delta_{a}, \alpha+\lambda \delta_{j}\right) z^{\left(\delta_{a} \mid \alpha+\lambda \delta_{j}\right)} e^{\xi_{a}(t, z)} e^{-\eta_{a}(t, z)} \mathbb{X}^{\nu p, \sigma q}(y, w) \tau_{\alpha+\lambda \delta_{j}-\delta_{a}-\nu \delta_{p}-\sigma \delta_{q}}(x, t) e^{x z} \\
\otimes \mathbb{X}^{-a}(z) \tau_{\beta+\delta_{a}-\mu \delta_{k}}-\sum_{a=1}^{n} \epsilon\left(\delta_{a}, \alpha+\lambda \delta_{j}\right)(-z)^{-\left(\delta_{a} \mid \alpha+\lambda \delta_{j}\right)} \times \\
\left.e^{-\xi_{a}(t,-z)} e^{\eta_{a}(t,-z)} \mathbb{X}^{\nu p, \sigma q}(y, w) \tau_{\alpha+\lambda \delta_{j}+\delta_{a}-\nu \delta_{p}-\sigma \delta_{q}}(x, t) e^{x z} \otimes \mathbb{X}^{a}(-z) \tau_{\beta-\delta_{a}-\mu \delta_{k}}\right\} \\
+\mathbb{X}^{\nu p}(\nu y) \tau_{\alpha+\lambda \delta_{j}-\nu \delta_{p}}(x, t) \otimes \frac{\mathbb{X}^{\sigma q}(-\sigma w)}{w^{\delta} q 0} \tau_{\beta-\mu \delta_{k}-\sigma \delta_{q}}(x, t) \\
-\frac{\mathbb{X}^{\sigma q}(-\sigma w)}{w^{\delta q}} \tau_{\alpha+\lambda \delta_{j}-\sigma \delta_{q}}(x, t) \otimes \mathbb{X}^{\nu p}(\nu y) \tau_{\beta-\mu \delta_{k}-\nu \delta_{p}}(x, t) \\
=\frac{1}{2} \mathbb{X}^{\nu p, \sigma q}(y, w) \tau_{\alpha+\lambda \delta_{j}-\nu \delta_{p}-\sigma \delta_{q}}(x, t) \otimes \tau_{\beta-\mu \delta_{k}}(x, t) .
\end{gathered}
$$

Now divide by $\tau_{\alpha} \otimes \tau_{\beta}$ and rewrite terms like

$$
\begin{gathered}
\frac{e^{-\eta_{a}(t, z)} \mathbb{X}^{\nu p, \sigma q}(y, w) \tau_{a+\lambda \delta_{j}-\delta_{a}-\nu \delta_{p}-\sigma \delta_{q}}(x, t)}{\tau_{\alpha}(x, t)}= \\
e^{-\eta_{a}(t, z)}\left(\frac{\mathbb{X}^{\nu p, \sigma q}(y, w) \tau_{\alpha+\lambda \delta_{j}-\delta_{a}-\nu \delta_{p}-\sigma \delta_{q}}(x, t)}{\tau_{a+\lambda \delta_{j}-\delta_{a}}(x, t)}\right) \frac{e^{-\eta_{a}(t, z)} \tau_{\alpha+\lambda \delta_{j}-\delta_{a}}(x, t)}{\tau_{a}(x, t)} .
\end{gathered}
$$

Remove the tensor symbols $\otimes$ and write $x^{\prime}, t^{\prime}$ etc. for the second component of the tensor 
product. Then (6.18) is equivalent to

$$
\begin{aligned}
& \operatorname{Res}_{z=0} d z\left\{\frac{1-\epsilon}{2 z} \frac{\mathbb{X}^{\nu p, \sigma q}(y, w) \tau_{\alpha+\lambda \delta_{j}-\nu \delta_{p}-\sigma \delta_{q}}(x, t)}{\tau_{\alpha+\lambda \delta_{j}}(x, t)} \Psi^{+}(\alpha, x, t, x)_{\lambda j, 0} \Psi^{-}\left(\beta, x^{\prime}, t^{\prime}, z\right)_{\mu k, 0}\right. \\
& +\frac{\epsilon}{2 z} e^{-\eta_{0}(t, z)}\left(\frac{\mathbb{X}^{\nu p, \sigma q}(y, w) \tau_{\alpha+\lambda \delta_{j}-\nu \delta_{p}-\sigma \delta_{q}(x, t)}}{\tau_{\alpha+\lambda \delta_{j}-\nu \delta_{p}}(x, t)}\right) \Psi^{+}(\alpha, x, t, z)_{\lambda j, 0} \Psi^{-}\left(\beta, x^{\prime}, t^{\prime}, z\right)_{\mu k, 0} \\
& +\sum_{a=1}^{n} e^{-\eta_{a}(t, z)}\left(\frac{\mathbb{X}^{\nu p, \sigma_{q}}(y, w) \tau_{\alpha+\lambda \delta_{j}-\delta_{a}-\nu \delta_{p}-\sigma \delta_{q}}(x, t)}{\tau_{a+\lambda \delta_{j}-\delta_{a}}(x, t)}\right) \Psi^{+}(\alpha, x, t, z)_{\lambda j, a} \Psi^{-}\left(\beta, x^{\prime}, t^{\prime}, z\right)_{\mu k, a}
\end{aligned}
$$

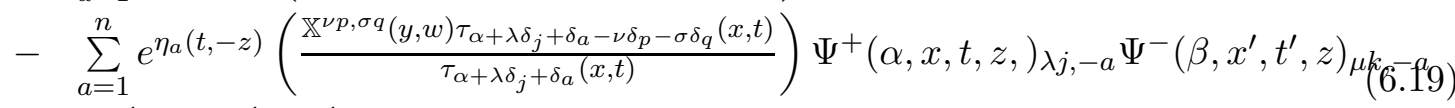

$$
\begin{aligned}
& +\frac{1}{(\sqrt{2})^{\delta_{p 0}}} \frac{1}{(\sqrt{2} w)^{\delta_{q 0}}} \frac{1}{z} \Psi^{+}(\alpha, x, t, y)_{\lambda j, \nu p} \Psi^{-}\left(\beta, x^{\prime}, t^{\prime}, w\right)_{\mu k,-\sigma q} \\
& \left.-\frac{1}{(\sqrt{2})^{\delta_{p, 0}}} \frac{1}{(\sqrt{2} w)^{\delta_{q 0}}} \frac{1}{z} \Psi^{+}(\alpha, x, t,-w)_{\lambda j, \sigma q} \Psi^{-}\left(\beta, x^{\prime}, t^{\prime},-y\right)_{\mu k,-\nu p}\right\} \\
& =\operatorname{Res}_{z=0} d z \frac{1}{2 z} \frac{\mathbb{X}^{\nu p, \sigma q}(y, w) \tau_{\alpha+\lambda \delta_{j}-\nu \delta_{p}-\sigma \delta_{q}}(x, t)}{\tau_{\alpha+\lambda \delta_{j}}(x, t)} \frac{\tau_{\alpha+\lambda \delta_{j}}(x, t)}{\tau_{\alpha}(x, t)} e^{x z} \frac{\tau_{\beta-\mu \delta_{k}}\left(x^{\prime}, t^{\prime}\right)}{\tau_{\beta}\left(x^{\prime}, t^{\prime}\right)} e^{-x^{\prime} z} .
\end{aligned}
$$

Now use (6.13) and the observation that

$$
\operatorname{Res}_{z=0} d z \frac{1}{z} f( \pm u)=\operatorname{Res}_{z=0} d z \pm \delta(u \mp z) f(z),
$$

then (6.19) turns into

$$
\begin{aligned}
& \operatorname{Res}_{z=0} d z E_{\lambda j, \lambda j}\left\{\left((1-\epsilon) \frac{\mathbb{X}^{\nu p, \sigma q}(y, w) \tau_{\alpha+\lambda \delta_{j}-\nu \delta_{p}-\sigma \delta_{q}}(x, t)}{\tau_{\alpha+\lambda \delta_{j}}(x, t)}\right.\right. \\
& \left.+\epsilon e^{-\eta_{0}(t, z)}\left(\frac{\mathbb{X}^{\nu p, \sigma q}(y, w) \tau_{\alpha+\lambda \delta_{j}-\nu \delta_{p}-\sigma \delta_{q}}(x, t)}{\tau_{\alpha+\lambda \delta_{j}}(x, t)}\right)\right) \Psi^{+}(\alpha, x, t, z) S(z) E_{00}{ }^{t} \Psi^{-}\left(\beta, x^{\prime}, t^{\prime}, z\right) \\
& +\sum_{a=1}^{n} e^{-\eta_{a}(t, z)}\left(\frac{\mathbb{X}^{\nu p, \sigma q}(y, w) \tau_{\alpha+\lambda \delta_{j}-\delta_{a}-\nu \delta_{p}-\sigma \delta_{q}}(x, t)}{\tau_{\alpha+\lambda \delta_{j}-\delta_{a}}(x, t)}\right) \Psi^{+}(\alpha, x, t, z) S(z) E_{a a}{ }^{t} \Psi^{-}\left(\beta, x^{\prime}, t^{\prime}, z\right) \\
& +\sum_{a=1}^{n} e^{\eta_{a}(t,-z)}\left(\frac{\mathbb{X}^{\nu p, \sigma q}(y, w) \tau_{\alpha+\lambda \delta_{j}+\delta_{a}-\nu \delta_{p}-\sigma \delta_{q}}(x, t)}{\tau_{\alpha+\lambda \delta_{j}+\delta_{a}}(x, t)}\right) \Psi^{+}(\alpha, x, t, z) S(z) E_{-a,-a}{ }^{t} \Psi^{-}\left(\beta, x^{\prime}, t^{\prime}, z\right) \\
& \left.+Z^{\nu p, \sigma q}(y, w) \Psi^{+}(\alpha, x, t, z){ }^{t} \Psi^{-}\left(\beta, x^{\prime}, t^{\prime}, z\right)\right\} \\
& =\operatorname{Res}_{z=0} d z E_{\lambda j, \lambda j} \frac{\mathbb{X}^{\nu p, \sigma q}(y, w) \tau_{\alpha+\lambda \delta_{j}-\nu \delta_{p}-\sigma \delta_{q}}(x, t)}{\tau_{\alpha+\lambda \delta_{j}}(x, t)} T^{+}(\alpha, x, t, z) \frac{E_{00}}{2 z}{ }^{t} T^{-}\left(\beta, x^{\prime}, t^{\prime}, z\right) .
\end{aligned}
$$

Next take $\beta=\alpha$ and define

$$
\begin{aligned}
& U_{\mu a}^{\lambda j}(z)=\sum_{k=0}^{\infty} U_{\mu a}^{\lambda j}(k) z^{-k}= \\
& \begin{cases}e^{-\mu \eta_{a}(t, z)\left(\frac{\mathbb{X}^{\nu p, \sigma q}(y, w) \tau_{a+\lambda \delta_{j}-\mu \delta_{a}-\nu \delta_{p}-\sigma \delta_{q}}(x, t)}{\tau_{a+\lambda \delta_{j}-\mu \delta_{a}}(x, t)}\right)} & \text { if } a \neq 0, \\
(1-\epsilon) \frac{\mathbb{X}^{\nu p, \sigma q}(y, w) \tau_{\alpha+\lambda \delta_{j}-\nu \delta_{p}-\sigma \delta_{p}}(x, t)}{\tau_{\alpha+\lambda \delta_{j}}(x, t)}+ & \text { if } a=0 . \\
\epsilon e^{-\eta_{0}(t, z)\left(\frac{\mathbb{X}^{\nu, \sigma q}(y, w) \tau_{\alpha+\lambda \delta_{j}-\nu \delta_{p}-\sigma \delta_{q}}(x, t)}{\tau_{\alpha+\lambda \delta_{j}}(x, t)}\right)} & \end{cases}
\end{aligned}
$$


Again we assume that $\mu=+$ when $a=0$. Now using corollary 5.1 we deduce from (6.20) that

$$
\begin{aligned}
& E_{\lambda j, \lambda j}\left(Z^{\nu p, \sigma q}(\alpha, y, w) P^{+}(\alpha) S(\partial) P^{-}(\alpha)^{*}+\sum_{a=-n}^{n} \sum_{k=0}^{\infty} U_{a}^{\lambda j}(k) P^{+}(\alpha) E_{a a} \partial^{-k} S(\partial) P^{-}(\alpha)^{*}\right)_{-} \\
= & E_{\lambda j \lambda j} \frac{\mathbb{X}^{\nu p, \sigma q}(y, w) \tau_{a+\lambda \delta_{j}-\nu \delta_{p}-\sigma \delta_{q}}(x, t)}{\tau_{a+\lambda \delta_{j}}(x, t)} T^{+}(\alpha) \frac{E_{00} \partial^{-1}}{2}{ }^{t} T^{-} \alpha .
\end{aligned}
$$

Substitute (5.8) and one obtains.

$$
\begin{aligned}
& E_{\lambda j, \lambda j}\left(Z^{\nu p, \sigma q}(\alpha, y, w) T^{+}(\alpha) S(\partial){ }^{t} T^{-}(\alpha)+\right. \\
+ & \left.\sum_{a=-n}^{n} \sum_{k=0}^{\infty} U_{a}^{\lambda j}(k) P^{+}(\alpha) E_{a a} \partial^{-k} P^{+}(\alpha)^{-1} T^{+}(\alpha) S(\partial){ }^{t} T^{-}(\alpha)\right)_{-} \\
= & E_{\lambda j, \lambda j} \frac{\mathbb{X}^{\nu p, \sigma q}(y, w) \tau_{\alpha+\lambda \delta_{j}-\nu \delta_{p}-\sigma \delta_{q}}(x, t)}{\tau_{\alpha+\lambda \delta_{j}}(x, t)} T^{+}(\alpha) \frac{E_{00} \partial^{-1}}{2}{ }^{t} T^{-}(\alpha) .
\end{aligned}
$$

We can mulitply from the right with $\left({ }^{t} T(\alpha)^{-}\right)^{-1}$, then the above formula is equivalent to

$$
\begin{gathered}
E_{\lambda j, \lambda j} T^{+}(\alpha)\left(Y^{\nu p, \sigma q}(\alpha, y, w)_{<}+\sum_{a=-n}^{n} \sum_{k=0}^{\infty} U_{a}^{\lambda j}(k)\left(K^{+}(\alpha) E_{a a} \partial^{-k}\left(K^{+}(\alpha)\right)^{-1}\right)_{<}\right. \\
\left.-\frac{\mathbb{X}^{\nu p, \sigma q}(y, w) \tau_{a+\lambda \delta_{j}-\nu \delta_{p}-\sigma \delta_{q}}(x, t)}{\tau_{\alpha+\lambda \delta_{j}}(x, t)} E_{00}\right)=0 .
\end{gathered}
$$

Now notice that

$$
\begin{aligned}
& E_{00} T(\alpha)^{+}=E_{00}, \\
& E_{\lambda j, \lambda j} T^{+}(\alpha)=E_{\lambda j, \lambda j}+\frac{\tau_{\alpha+\lambda \delta_{j}}(x, t)}{\tau_{\alpha}(x, t)} E_{\lambda j, 0},
\end{aligned}
$$

from which one concludes that

$$
\begin{gathered}
E_{\lambda j, \lambda j}\left(Y^{\nu p, \sigma q}(\alpha, y, w)_{<}+\sum_{a=-n}^{n} \sum_{k=0}^{\infty} U_{a}^{\lambda j}(k)\left(K^{+}(\alpha) E_{a a} \partial^{-k}\left(K^{+}(\alpha)\right)^{-1}\right)_{<}\right. \\
\left.-\frac{\mathbb{X}^{\nu p, \sigma q}(y, w) \tau_{\alpha+\lambda \delta_{j}-\nu \delta_{p}-\sigma \delta_{q}}(x, t)}{\tau_{\alpha+\lambda \delta_{j}}(x, t)} E_{00}\right)=0 .
\end{gathered}
$$

Finally, act with the left-hand-side of formula (6.21) on $\Phi^{+}(\alpha, x, t, z)$ and one obtains the OSASM-formula.

Theorem 6.1 Let $0 \leq j \leq n, \lambda=+,-$ and $\lambda=+$ when $j=0$, moreover assume that $\delta_{0}=0$. Then one has the following OSASM-formula for the BKP hierarchy:

$$
\begin{aligned}
& E_{\lambda j, \lambda j} Y^{\nu p, \sigma q}(\alpha, y, w)_{<} \Phi^{+}(\alpha, x, t, z)= \\
& -E_{\lambda j, \lambda j}\left(\left(1+\epsilon\left(e^{-\eta_{0}(t, z)}-1\right)\right)\left(\frac{\mathbb{X}^{\nu p, \sigma q}(y, w) \tau_{\alpha+\lambda \delta_{j}-\nu \delta_{p}-\sigma \delta_{q}}(x, t)}{\tau_{\alpha+\lambda \delta_{j}}(x, t)}\right) \Phi^{+}(\alpha, x, t, z) E_{00}\right. \\
& \left.-\frac{\mathbb{X}^{\nu p, \sigma q}(y, w) \tau_{\alpha+\lambda \delta_{j}-\nu \delta_{p}-\sigma \delta_{q}}(x, t)}{\tau_{\alpha+\lambda \delta_{j}}(x, t)} E_{00} \Phi^{+}(\alpha, x, t, z)\right)+ \\
& +\sum_{a=1}^{n}\left(e^{-\eta_{a}(t, z)}\left(\frac{\mathbb{X}^{\nu p, \sigma q}(y, w) \tau_{\alpha+\lambda \delta_{j}-\delta_{a}-\nu \delta_{p}-\sigma \delta_{q}}(x, t)}{\tau_{\alpha+\lambda \delta_{j}-\delta_{a}}(x, t)}\right) \Phi^{+}(\alpha, x, t, z) E_{a a}\right. \\
& \left.\left.-\frac{\mathbb{X}^{\nu p, \sigma q}(y, w) \tau_{\alpha+\lambda \delta_{j}-\delta_{a}-\nu \delta_{p}-\sigma \delta_{q}}(x, t)}{\tau_{\alpha+\lambda \delta_{j}-\delta_{a}}(x, t)}\right) E_{a a} \Phi^{+}(\alpha, x, t, z)\right) \\
& +\sum_{a=1}^{n}\left(e^{\eta_{a}(t,-z)}\left(\frac{\mathbb{X}^{\nu p, \sigma q}(y, w) \tau_{\alpha+\lambda \delta_{j}+\delta_{a}-\nu \delta_{p}-\sigma \delta_{q}}(x, t)}{\tau_{\alpha+\lambda \delta_{j}+\delta_{a}}(x, t)}\right) \Phi^{+}(\alpha, x, t, z) E_{-a,-a}\right. \\
& \left.-\frac{\mathbb{X}^{\nu p, \sigma q}(y, w) \tau_{\alpha+\lambda \delta_{j}+\delta_{a}-\nu \delta_{p}-\sigma \delta_{q}}(x, t)}{\tau_{\alpha+\lambda \delta_{j}+\delta_{a}}(x, t)} E_{-a,-a} \Phi^{+}(\alpha, x, t, z)\right) .
\end{aligned}
$$




\section{Acknowledgements}

Part of this research was done while JvdL was financially supported by the "Stichting Fundamenteel Onderzoek der Materie (F.O.M.). During that time he also visited MIT. He would like to thank the Mathematics Department of MIT for the kind hospitality.

\section{References}

[1] M. Adler, T. Shiota and P. van Moerbeke, From the $w_{\infty}$-algebra to its central extension: a $\tau$-function approach, Physics Letters A, 194 (1994), 33-34.

[2] M. Adler, T. Shiota and P. van Moerbeke, A Lax representation of the vertex operator and the central extension, Comm. Math. Phys. 171 (1995), 547-588.

[3] E. Date, M. Jimbo, M. Kashiwara and T. Miwa, Operator approach to the KadomtsevPetviashvili equation. Transformation groups for soliton equations. III, J. Phys. Soc. Japan 50 (1981), 3806-3812.

[4] E. Date, M. Jimbo, M. Kashiwara and T. Miwa, Transformation groups for soliton equations IV, A new hierarchy of soliton equations of KP type, Physica 4D (1982), 343-365.

[5] I. Frenkel, J. Lepowsky and A. Meurman, Vertex operator algebras and the monster, Academic Press, 1989.

[6] M. Jimbo and T. Miwa, Solitons and infinite dimensional Lie algebras, Publ. Res. Inst. Math. Sci. 19 (1983), 943-1001.

[7] V.G. Kac , Infinite dimensional Lie algebras, Progress in Math., vol. 44, Brikhäuser, Boston, 1983; 2nd ed., Cambridge Univ. Press, 1985; 3d ed., Cambridge Univ. Press, 1990 .

[8] V.G. Kac , Vertex algebras for beginners, University lecture series 10, AMS., Providence, RI, 1996.

[9] V.G. Kac and D.H. Peterson, 112 constructions of the basic representation of the loop group of $E_{8}$ in Proc. of the Symposium "Anomalies, Geometry, Topology", Argonne, eds. W.A. Bardeen, A.R. White, World Scientific, 1985, pp. 276-298.

[10] V.G. Kac and A.K. Raina, Bombay lectures on highest weight representations of infinite-dimensional Lie algebras, Advanced Ser. in Math. Phys., vol. 2, World Scientific 1987.

[11] V.G. Kac and J.W. van de Leur, The n-component KP hierarchy and representation theory in "Important developments in soliton theory", eds. A.S. Fokas and V.E. Zakharov, Springer series in Nonlinear Dynamics, Berlin Heidelberg, 1993. In the second edition of this book, which will be published soon, there is an extended version of this paper. We refer to this second edition.

[12] M. Mulase, Solvability of the Super KP equation and a generalization of the Birkhoff decomposition, Invent. Math. 92 (1988), 1-46. 
[13] M. Mulase, Complete integrability of the Kadomtsev-Petviashvili equation, Advances in Math. 54 (1984), 57-66.

[14] A.Yu. Orlov and E.I. Schulman, Additional symmetries for integrable and conformal algebra representations, Lett. Math. Phys. 12 (1986), 171.

[15] M. Sato Soliton equations as dynamical systems on infinite dimensional Grassmann manifolds, Res. Inst. Math. Sci. Kokyuroku 439 (1981), 30-46.

[16] T. Shiota, Prym varieties and soliton equations in "Infinite dimensional Lie algebras and groups", ed. V.G. Kac, Advanced Ser. in Math. Phys., vol. 7, World Scientific 1989, 407-448.

[17] F. ten Kroode and J. van de Leur, Bosonic and fermionic realizations of the affine algebra $\hat{g l}_{n}$, Comm. Math. Phys. 137 (1991), 67-107.

[18] F. ten Kroode and J. van de Leur, Bosonic and fermionic realizations of the affine algebra $\hat{s o}_{2 n}$, Commun. in Algebra 20 (1992), 3119-3162.

[19] J.W. van de Leur, The $W_{1+\infty}\left(g l_{s}\right)$-symmetries of the $s$-component KP hierarchy, Journal of Math. Phys. 37 (1996), 2315-2337. 